Supporting Information

\title{
Per- and Polyfluoroalkyl Substances in Contaminated Soil and Groundwater at Airports: a Canadian Case Study
}

\author{
Min Liu', Gabriel Munoz ${ }^{1,2}$, Sung Vo Duy², Sébastien Sauvé2*, and Jinxia Liu ${ }^{1 *}$ \\ ${ }^{1}$ Department of Civil Engineering, McGill University, Montreal, QC H3A 0C3, Canada. \\ ${ }^{2}$ Department of Chemistry, Université de Montréal, Montreal, QC H3C 3J7, Canada.
}

*Corresponding authors: jinxia.liu@mcgill.ca; sebastien.sauve@umontreal.ca 


\section{Table of Contents}

Per- and Polyfluoroalkyl Substances in Contaminated Soil and Groundwater at Airports: a Canadian Case

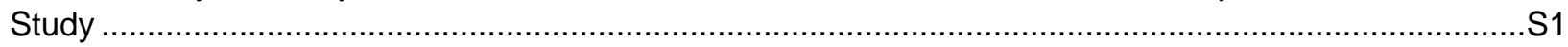

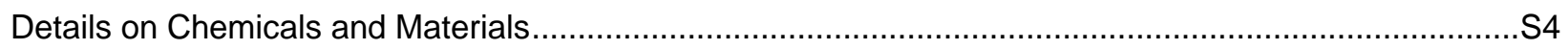

Field soil and groundwater sample preparation without persulfate oxidation .........................................S5

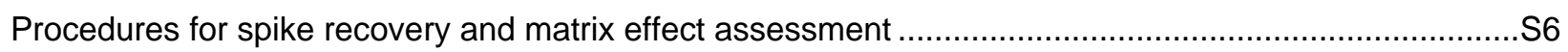

Method validation of total oxidizable precursor (TOP) assay for soils ...................................................... 8

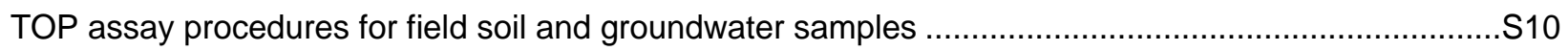

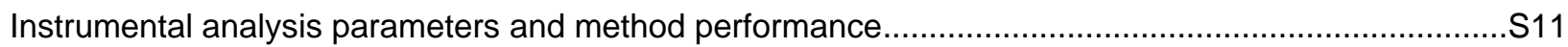

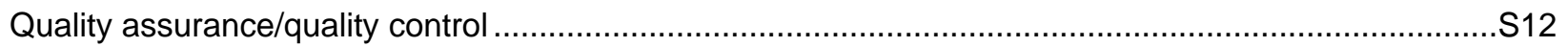

Rationales for associating quantification confidence levels ............................................................ 13

\section{List of Tables}

Table S1. Ion formula, theoretical and observed $\mathrm{m} / \mathrm{z}$, mass error, retention time (RT) and commercial sources of 53 native PFASs with available standards.

Table S2. Acronym, full name, theoretical and observed m/z, RT and commercial sources of isotope-labeled IS.

Table S3. Detailed information about field soil and groundwater samples. ..S18

Table S4. The property and PFAS background levels of soils used for spike recovery test, matrix effect assessment, and TOP method validation purposes.

S20

Table S5. The spike recovery (mean \pm SE, \%) and matrix effects (mean \pm SE, \%) of 53 quantitative PFAS analytes in three types of soil matrixes.

Table S6. The spike recoveries (mean \pm SE, \%) of 53 quantitative PFAS in three types of soils with the new exhaustive extraction method.

Table S7. PFAS analyte list (target and suspect-screening) for field soil and groundwater samples. .....S26

Table S8. Correspondence between native PFAS analytes and isotopically labeled surrogate IS..........S39

Table S9. The acronym, theoretical and observed $\mathrm{m} / \mathrm{z}$, mass error, RT, analysis method and identification confidence level of suspect-screening PFAS.

Table S10. Compound-specific instrumental limits of detection (iLOD), instrumental limits of quantification (iLOQ), method limits of detection ( $\mathrm{mLOD}$ ), and limits of quantification ( $\mathrm{mLOQ}$ ) and linearity performance of 53 quantitative PFAS in soil and groundwater. S43

Table S11. The median PFHxS/PFOS ratio in surface soil and groundwater at different areas of four FTA sites. S45

Table S12. A draft priority PFAS analyte list for surface soil (a) and groundwater (b). S46 


\section{List of Figures}

Figure S1. Sampling locations for soil and groundwater samples from the four FTA sites, and site history.

Figure S2. Recovery of PFAS during the freeze-drying step, in soil matrix. Error bars represent standard deviations. S50

Figure S3. The PFAA recovery in three types of soils during the whole TOP procedure. S51

Figure S4. The molar conversion yields of five FT-based and three ECF-based precursors into C3-C10 PFCA post TOP assay in three types of soil matrixes (1R, 2N and $3 \mathrm{~F}$ soil) and ultra-pure water; the precursors included 6:2 FTSA (a), 8:2 FTSA (b), 6:2 FTAB (c), 5:3 FTB (d), 5:1:2 FTB (e), FHxSA (f), PFHxSAm (g), and PFHxSAmS (h). S52

Figure S5. The structure of 53 quantitative PFAS. .S53

Figure S6. The concentrations of five types of PFAS in surface soils (a) and groundwater (b) from the four Canadian FTA sites. S54

Figure S7. The fifteen highest PFAS measured in AFFF-impacted soils (a-d) and groundwater (e-h) in the vicinity of FTA area at the four FTA sites. The zwitterionic and cationic precursors were marked with a red asterisk $\left({ }^{*}\right)$. .S55

Figure S8. The PFAA concentrations in surface soil (a) and groundwater (b) samples from the four Canadian FTA sites. S56

Figure S9. The profiles of ECF-derived sulfonamides in surface soil (a) and groundwater (b) samples from the four Canadian FTA sites. S57

Figure S10. Likely in-situ transformation pathways of FT-derived precursors in source zone soils (a) and groundwater (c) of Site \#1 and the concentrations of the precursor and transformation products in soil (b) and groundwater (d) samples. Likely in-situ transformation pathways of ECF-derived sulfonamides in source zone soils of Site \#1 (e) and the concentrations of the precursor and transformation products in all samples (f). S58

Figure S11. The profiles of FT-derived compounds in surface soil (a) and groundwater (b) samples from the four Canadian FTA sites. S60

Figure S12. The changes in the concentrations of 15 PFAS (mainly detected in surface soils) over depths at five sampling locations (a) 4S, (b)2S, (c) 6S, (d)7S, (e) 5S at site \#3. The five sample locations are shown in the scheme map $(\mathrm{f})$.

S61

Figure S13. The molar concentration of both known and total precursors in (a-c) surface soil and (d-g) groundwater samples at the four Canadian FTA sites. The left bar represents the molar concentration of known precursors identified via UHPLC-HRMS through the target and suspect-screening methods, while the right bar shows the molar concentration of total precursors determined by TOP assay. The C3-C15 represented the carbon numbers of the known precursors (left bars) and the carbon number of PFCA produced from the precursors (right bars). S62

Figure S14. The molar fraction of unknown precursors in $\Sigma$ PFAS in both surface soil (a) and groundwater (b) when assuming molar PFCA yields of $80 \%, 100 \%$ and $120 \%$ from TOP. S64

Reference. .S65 


\section{Details on Chemicals and Materials}

HPLC-water, HPLC-water containing $0.1 \%$ formic acid, methanol, and acetonitrile were of LC-MS grade and were obtained from Fisher Scientific (Whitby, ON, Canada). Ammonium acetate (purity $\geq 98 \%$ ), sodium hydroxide (pellets, purity $\geq 97 \%$ ), ammonium hydroxide (25-30\% in water), and formic acid (reagent grade, purity $\geq 95 \%$ ) were acquired from Sigma-Aldrich (St. Louis, MO, USA). Hydrochloric acid (35-38\% in water), and glacial acetic acid were obtained from Fisher Scientific (Whitby, ON, Canada). Nitrogen $\left(\mathrm{N}_{2}\right)$ (purity 99.998\%) was from MEGS Inc. (St-Laurent, QC, Canada). Superclean ENVI-Carb cartridges (250 mg/6 mL) were obtained from Supelco (Bellefonte, PA, USA).

The sources of 53 PFAS with available native standards are shown in Table S1. The native PFASs obtained from Wellington Labs, Inc. (Whitby, ON, Canada) had chemical purities $>98 \%$. These standards were either acquired at $2 \mu \mathrm{g} \mathrm{mL}^{-1}$ (as compound or salt) as mixtures or separately at $50 \mu \mathrm{g} \mathrm{mL}^{-1}$ (as compound or salt) as individual compounds.

Isotope-labeled internal standards (IS) (see Table S2) were all obtained from Wellington Labs, Inc. (Whitby, ON, Canada). Perfluorooctane amidoakyl ammonium salt (PFOAAmS) was custom synthesized at Beijing Surfactant Institute (Peking, China) and was used as an internal standard for positive mode native analytes. ${ }^{13} \mathrm{C}_{4}$-PFBA, ${ }^{13} \mathrm{C}_{5}$-PFPeA, ${ }^{13} \mathrm{C}_{5}$-PFHXA, ${ }^{13} \mathrm{C}_{4}$-PFHpA, ${ }^{13} \mathrm{C}_{8}$-PFOA, ${ }^{13} \mathrm{C}_{9}$-PFNA, ${ }^{13} \mathrm{C}_{6}$-PFDA, ${ }^{13} \mathrm{C}_{7}-\mathrm{PFUnA},{ }^{13} \mathrm{C}_{2-}$ PFDoA, ${ }^{13} \mathrm{C}_{2}$-PFTeDA, ${ }^{13} \mathrm{C}_{2}$-PFHxDA, ${ }^{13} \mathrm{C}_{3}$-PFBS, ${ }^{13} \mathrm{C}_{3}$-PFHxS, ${ }^{13} \mathrm{C}_{8}$-PFOS, ${ }^{13} \mathrm{C}_{8}$-FOSA, $d_{3}-\mathrm{MeFOSA}, d_{5}-$ EtFOSA, $d_{3}$-MeFOSAA, ${ }^{13} \mathrm{C}_{2}-6: 2-\mathrm{FtS},{ }^{13} \mathrm{C}_{2}-8: 2-\mathrm{FtS},{ }^{13} \mathrm{C}_{2}-6: 2-\mathrm{FTUA},{ }^{13} \mathrm{C}_{2}-8: 2-\mathrm{FTUA}$, and PFOAAmS were the internal standards used in the present study. The association between the native analyte and internal standard is provided in Table S8. 


\section{Field soil and groundwater sample preparation without persulfate oxidation}

Soils. The method modified from previous studies was applied for the field soil samples. ${ }^{1}$ Specifically, $1 \mathrm{~g}$ $(\mathrm{dw})$ of soil for each sample was weighed in $15-\mathrm{mL}$ polypropylene (PP) tubes (previously cleaned with $\mathrm{MeOH})$. Then $5 \mu \mathrm{g} / \mathrm{kg} \mathrm{dw}$ of surrogate internal standard solution mixture $\left(100 \mu \mathrm{L}, 50 \mathrm{ng} \mathrm{mL}^{-1}\right)$ was spiked into each soil sample. After a wait time of $\sim 60 \mathrm{~min}$, soil samples were submitted to three sequential solvent extraction cycles. In each cycle, the soil was extracted with $4 \mathrm{~mL}$ of $100 \mathrm{mM}$ of ammonium acetate (AmmoAce) in methanol, vortexed for $0.5 \mathrm{~min}$, ultrasonicated for $10 \mathrm{~min}$, and subjected to centrifugation (5000 rpm, $5 \mathrm{~min}$ ). The supernatant was transferred into a new 15-mL PP tube.

The combined extract $(\sim 12 \mathrm{~mL})$ was then subjected to cleanup. The extract was transferred onto an ENVICarb graphite cartridge $(250 \mathrm{mg} / 6 \mathrm{~mL}$, pre-cleaned with $4 \mathrm{~mL}$ of $\mathrm{MeOH})$, and the eluate was directly recovered in a new $15-\mathrm{mL}$ PP tube. The tubes containing soil extracts were also rinsed with $0.5 \mathrm{~mL}$ of $\mathrm{MeOH}$ and passed through the cartridges. The cartridges were rinsed with $1 \mathrm{~mL}$ of $\mathrm{MeOH}$ in the end. The resulting extract was concentrated using a gentle stream of $\mathrm{N}_{2}$ and mild heating $\left(40^{\circ} \mathrm{C}\right)$ and finally adjusted to a volume of $2 \mathrm{~mL}$. Following brief vortexing $(0.25 \mathrm{~min})$, a $150-\mu \mathrm{L}$ aliquot of sample was introduced into a $250-\mu \mathrm{L}$ polypropylene HPLC vial, along with $50 \mu \mathrm{L}$ of a $20 \mathrm{ng} \mathrm{mL}^{-1}$ injection internal standard solution mixture (MPFAC-C-IS from Wellington Labs). Following brief vortexing $(0.1 \mathrm{~min})$, the extracts were submitted to UHPLC-HRMS analysis. For those PFAS present at very high concentrations, new sample preparation was performed if necessary to fall within linear working range.

Groundwater. Groundwater samples were prepared as follows. After gently inverting the bottles for homogenization (but avoiding foaming), a $40-\mathrm{mL}$ aliquot of sample from the original collection bottle was collected from $\sim 10 \mathrm{~cm}$ below the air-water interface, introduced in a 50-mL PP tube, and submitted to centrifugation (5 $\mathrm{min} ; 6000 \mathrm{rpm}$ ). A 41.7- $\mu \mathrm{L}$ aliquot of groundwater was then transferred to a polypropylene tube, to which $28.3 \mu \mathrm{L}$ of HPLC-water, $140 \mu \mathrm{L}$ of $\mathrm{MeOH}$, and $140 \mu \mathrm{L}$ of a $6.25 \mathrm{ng} \mathrm{mL}^{-1}$ internal standard solution (prepared in $\mathrm{MeOH}$ ) were added. The sample was briefly vortexed and a $200-\mu \mathrm{L}$ aliquot of sample was transferred to a 250- $\mu \mathrm{L}$ polypropylene HPLC vial. The final extract composition was $80: 20 \mathrm{MeOH}$ :water $(\mathrm{v} / \mathrm{v})$. Note that internal calibration curves were built accordingly $(80: 20 \mathrm{MeOH}: H P L C$-water $\mathrm{v} / \mathrm{v})$ with the same IS concentration. The dilution factor of $8.4 \times$ was considered to derive the actual concentration. 


\section{Procedures for spike recovery and matrix effect assessment}

The procedures for the determination of whole-method spike recovery and instrumental matrix effect assessment were adapted from previous studies. ${ }^{2,3}$

\section{Validation of the method spike recovery}

Three soils were obtained locally from areas without known PFAS point sources (Table S2), and were used to evaluate the spike recovery of the final retained method (extraction using $100 \mathrm{mM} \mathrm{CH}_{3} \mathrm{COONH}_{4}$ in $\mathrm{MeOH}$, Envicarb cartridge cleanup, and evaporation). The soil samples ( $n=3$ per soil matrix) were spiked with 10 $\mu \mathrm{g} / \mathrm{kg} \mathrm{dw}$ of native standards (i.e., $100 \mu \mathrm{L}$ of a mixture containing the 53 certified PFAS at $100 \mathrm{ng} \mathrm{mL}^{-1}$ in $\mathrm{MeOH}$ ) and then processed using the procedures as described above. These samples were referred to as "spiked before" (SB). In parallel, for each soil type, six non-spiked soil samples were added with $200 \mu \mathrm{L}$ of $\mathrm{MeOH}$ and processed as per the same procedure. At the end of the preparation procedure, three of the latter samples were spiked with $10 \mathrm{ng} / \mathrm{g}$ of native standards (referred to as "spiked after" [SA] samples), while the other three were left unspiked (referred to as "non-spiked" [NS] samples). The three sets of samples (i.e. SB, SA, and NS) were then spiked with internal standards, briefly vortexed, and analyzed by HPLC-MS. The spike recovery was determined as per the following equation: ${ }^{3}$

Recovery $(\%)=100 * \frac{S B-N S}{S A-N S} \quad$ (Equation 1)

where 'SB' is the native analyte to internal standard area ratio observed in a sample spiked before extraction with native analytes, 'SA' is the native analyte to internal standard area ratio observed in a sample spiked at the end of the analytical procedure with native analytes, and 'NS' is the native analyte to internal standard area ratio of the non-spiked sample.

The recovery test for soil sample preparation gave satisfactory recovery for the 53 quantitative PFAS with available standards (in the range of $60 \%-140 \%$ ), validating the method efficiency. However, trifluoroacetic acid (TFA) and perfluorooctadecanoic acid (PFOcDA) were not quantified due to instrument limitations.

\section{Assessment of the instrumental matrix effect}

The same three soils were used to examine potential matrix effects at the UHPLC-MS analysis stage. For each soil type, the matrix effect at the instrumental analysis stage was evaluated by comparing aliquots of soil extract spiked post-preparation (extraction, cleanup, and concentration) to that in a matrix-free (solventbased) reference.

Two types of matrix effects were investigated. The absolute matrix effect (Equation 2) is determined based on the native analyte absolute area, while the effective matrix effect refers to that evaluated based on the native analyte to internal standard area ratio (Equation 3):

Absolute matrix effect $(\%)=100 *\left(\frac{M-N S}{S}-1\right) \quad$ (Equation 2) 
where ' $M$ ' is the native analyte absolute area in the spiked soil matrix, 'NS' is the native analyte absolute area in the non-spiked soil matrix, and ' $S$ ' is the native analyte absolute area in the spiked matrix-free reference.

Effective matrix effect $(\%)=100 *\left(\frac{m-n s}{s}-1\right) \quad$ (Equation 3)

where ' $m$ ' is the native analyte to internal standard area ratio in the spiked soil matrix, ' $\mathrm{s}$ ' is the native analyte to internal standard area ratio in the non-spiked soil matrix, and ' $s$ ' is the native analyte to internal standard area ratio in the spiked matrix-free reference. 


\section{Method validation of total oxidizable precursor (TOP) assay for soils}

The same three soils (Table S2) were also used for TOP method validation, with all treatments in triplicate for each type of soil. The soil sample preparation method before TOP was modified from Nickerson et al. ${ }^{4}$ In detail, $1 \mathrm{~g} \mathrm{dw}$ of soil was weighed into a pre-cleaned PP tube and then spiked with $67 \mu \mathrm{l}$ of an individual precursor solution (6:2 FTSA, 8:2 FTSA, 6:2 FTAB, FHxSA, PFHxSAm or PFHxSAmS methanolic solution at a concentration of $17.91 \mathrm{ppm})$. After 1 -h stabilization, the soils were extracted with a basic solvent $(\mathrm{MeOH}$ with $0.4 \mathrm{M} \mathrm{NaOH}$ ) for two cycles, followed by an acidic solvent ( $\mathrm{MeOH}$ with $0.4 \mathrm{M} \mathrm{HCl}$ ) for one cycle. Each extraction cycle consisted of high-speed vortexing for $30 \mathrm{~s}$, ultrasonication for $10 \mathrm{~min}$, and centrifugation at $6000 \mathrm{rpm}$ for $5 \mathrm{~min}$. The supernatants of the first two cycles were combined $(4 \mathrm{ml})$, while the supernatant from the third cycle $(2 \mathrm{ml})$ was separately collected. Then the basic extracts $(4 \mathrm{ml})$ and acid extracts $(2 \mathrm{ml})$ were sequentially passed through Envi-Carb graphite cartridges $(6 \mathrm{~mL} / 250 \mathrm{mg})$ for cleanup and stored in separate PP tubes. The two fractions of cleanup extracts were combined $(10 \mathrm{ml})$, neutralized with $\mathrm{HCl}$ (adjust $\mathrm{pH}$ to 7), and adjusted to a final volume of $11 \mathrm{~mL}$. After centrifugation (5000 rpm, $5 \mathrm{~min}$ ), an aliquot $(1 \mathrm{ml})$ of the supernatant was transferred to a $15-\mathrm{mL}$ HDPE tube and evaporated to dryness at $45^{\circ} \mathrm{C}$.

The TOP assay procedure was modified from Houtz and Sedlak's. ${ }^{5}$ Specifically, $3.6 \mathrm{~mL}$ of water was added into the 15-mL centrifuge tube with dried soil extract, sonicated for $20 \mathrm{~min}$, then $1968 \mu \mathrm{L}$ of potassium persulfate at $175 \mathrm{mM}$ in HPLC-water was added (final concentration of $60 \mathrm{mM}$ ), followed by the addition of $172.2 \mu \mathrm{L}$ of $5 \mathrm{M} \mathrm{NaOH}$ (a final concentration of $150 \mathrm{mM}$ ). After mixing, the tubes with the aqueous solution were placed into a heated water bath $\left(85^{\circ} \mathrm{C}\right)$ for 6 hours. At the end of the reaction, the tubes were removed from the water bath and a wait time was applied to let the samples cool down to room temperature. Subsequently, $30 \mu \mathrm{L}$ of $\mathrm{HCl} 6 \mathrm{M}$ and $300 \mu \mathrm{L}$ of methanol were sequentially added, and the capped tubes were inverted for mixing. After that, $70 \mathrm{uL}$ of TOP sample, $140 \mathrm{uL}$ of internal standard solution at $6.25 \mathrm{ppb}$ (in $\mathrm{MeOH}$ ), and $140 \mathrm{uL}$ of methanol were added into a separate vial $(2 \mathrm{~mL})$. After brief vortexing and centrifugation (to separate out the salt precipitate), a $180-\mu \mathrm{L}$ aliquot of the supernatant was transferred to an injection vial for instrument analysis.

The tubes with the three soil matrixes without PFAS spike were processed in parallel to the spiked soils for subtracting the initial PFCAs extracted/generated from the background soil matrix.

The TOP assay was also performed on the precursors in HPLC water; reference tubes were spiked with an equivalent amount of precursor and subjected to the TOP procedure. The PFCA oxidation yields in soil and ultrapure water matrixes were thus compared. 


\section{TOP assay performance - verification of volatile loss and sorption loss}

The potential PFCA loss from the TOP procedure (including adsorption to vials, tubes, and volatilization) was checked to ensure the accuracy of the TOP assay result. Figure S2 illustrates the PFAA recovery in three types of soils during the whole TOP procedure. Both PFCA (C3-C9) and PFSA (C4, C6, C7, and C8) showed acceptable recovery, ranging from $60.2 \%$ to $127.8 \%$, during the soil sample preparation and TOP procedure. This indicates a minimal influence of adsorption to vials, tubes, and volatilization on PFAA losses during TOP. Given that the PFCA background levels from soil preparation and TOP procedures (procedural blank) and the PFCA background levels in soil matrixes could influence the determination of the PFCA yields, procedural blanks and soil matrix blanks (non-spiked soils) were submitted to the preparation and TOP procedure at the same time as the soil matrixes spiked with precursors. The procedural blanks showed nondetectable levels of each PFCA, while the PFCA background levels in soil matrixes were deducted when calculating the PFCA molar yields resulting from the spiked precursors.

\section{TOP assay performance - verification of oxidation yields}

Implementation of the TOP assay to soil requires efficient extraction of PFAS of various polarities, some of which form strong interactions with soil, preceding sample cleanup and persulfate oxidation. Method II achieved satisfactory spike recoveries for 53 target PFAS (70-99\%), except for FOSAA (62\%) in one soil (Table S6). The oxidation yields of 8 selected precursors (6:2 FTSA, 8:2 FTSA, 6:2 FTAB, 5:3 FTB, 5:1:2 FTB, FHxSA, PFHxSAm, and PFHxSAmS) were verified on $\mathrm{N}_{2}$-dried extracts of three types of soils with different textures and organic matter content (See the soil properties and PFAS background in Table S2). Results showed that $\mathrm{n}: 2$ fluorotelomers (FTs, including 6:2 FTAB, 6:2 FTSA, 8:2 FTSA) were completely consumed in both soils and ultra-pure water (as matrix-free control) during the TOP procedure (conversion ratio of 95.2-100\%), resulting in the production of PFCAs (chain length ranging from $C_{3}$ to $C_{n+1}$ ), with the dominance of $\mathrm{C}_{n-1}, \mathrm{C}_{\mathrm{n}-2}$, and $\mathrm{C}_{n}$ PFCA (Figure S4). The total PFCA yields from these 6:2 FTAB, 6:2 FTSA, and 8:2 FTSA in the tested soils fell between 63.3-92.3 mol\%, 74.2-98.1 mol\%, 66.0-84.9 mol\%, respectively.

The ECF-based $\mathrm{C}_{6}$ precursors (FHxSA, PFHxSAm, and PFHxSAmS) achieved total PFCA yields of 68.1$69.8 \mathrm{~mol} \%, 73.6-84.5 \mathrm{~mol} \%$, and $70.8-91.0 \mathrm{~mol} \%$, respectively, in the three soils (Figure S4). $\mathrm{C}_{6}$ PFCA (PFHxA) was the major product and $\mathrm{C}_{5}$ PFCA (PFPeA) was the minor product, which agrees with the production of $\mathrm{C}_{8}$ PFOA (major product) and $\mathrm{C}_{7}$ PFHpA (minor product) from ECF-based $\mathrm{C}_{8}$ precursors observed by Martin et al. in ultra-pure water. ${ }^{6}$ Though slightly biased-low, the PFCA yields in soils were consistent with those in HPLC-grade water tested in parallel, indicating acceptable oxidation efficiency. The low oxidative yield of FHxSA may be due to limited stability in the TOP aqueous medium or partial losses during the evaporation step. The PFCA yields were generally lowest for soil $2 \mathrm{~N}$, which contained more than $10 \%$ organic matter that may compete for hydroxyl radicals to render incomplete PFAS oxidation. ${ }^{7}$ Given the satisfactory PFCA yields from six selected precursors (except 5:3 FTB and 5:1:2 FTB), the validated TOP assay procedures were applied to the 45 soil samples for estimating unidentified precursors and total PFAS equivalent. 


\section{TOP assay procedures for field soil and groundwater samples}

Soil samples. The field soil samples were first extracted (MeOH with $0.4 \mathrm{M} \mathrm{NaOH}$ for 2 cycles followed by $\mathrm{MeOH}$ with $0.4 \mathrm{M} \mathrm{HCl}$ for 1 cycle), separately cleaned up on Envi-Carb cartridges, combined, and then the solvent extracts were evaporated to dryness. The dried soil extracts were subjected to a TOP procedure modified from Houtz and Sedlak's, ${ }^{5}$ as described in the section "Method validation of total oxidizable precursor (TOP) assay for soils".

Groundwater samples. For groundwater samples, the TOP procedures described by Martin et al. were used. ${ }^{6}$ In detail, a 1200- $\mu \mathrm{L}$ groundwater sample aliquot was added to a 2-mL centrifuge tube after centrifugation ( $5 \mathrm{~min} ; 6000 \mathrm{rpm}$ ). Following the addition of $656 \mu \mathrm{L}$ of $175 \mathrm{mM}$ potassium persulfate and 57.4 $\mu \mathrm{L}$ of $5 \mathrm{M} \mathrm{NaOH}$, the centrifuge tubes were placed in a water bath at $85^{\circ} \mathrm{C}$ for $6 \mathrm{~h}$. The samples were then removed from the bath and left to cool down to ambient temperature. The TOP medium was brought to $\mathrm{pH}$ $\sim 8$ with hydrochloric acid and amended with $100 \mu \mathrm{L}$ of $\mathrm{MeOH}$. After briefly vortexing the samples, a $70-\mu \mathrm{L}$ aliquot of oxidized sample was added to a polypropylene tube, along with $140 \mu \mathrm{L}$ of $\mathrm{MeOH}$ and $140 \mu \mathrm{L}$ of a $6.25 \mathrm{ng} / \mathrm{mL}$ internal standard solution (prepared in $\mathrm{MeOH}$ ). The sample was briefly vortexed, centrifuged (3 min; $6000 \mathrm{rpm}$ ), and a $200 \mu \mathrm{L}$ aliquot of sample was transferred to a $250-\mu \mathrm{L}$ polypropylene HPLC vial. Internal calibration curves (i.e., solvent-based: 80:20 MeOH: HPLC-water v/v) were used for quantification purposes after verifying the lack of matrix effects in the presence of the methanol diluted TOP medium. ${ }^{6}$ The procedure derived the same dilution factor of $8.4 \times$ as that of the samples analyzed without persulfate oxidation. 


\section{Instrumental analysis parameters and method performance}

Details on UHPLC-MS operating parameters. The Dionex Ultimate 3000 LC was controlled via the Chromeleon 7.2 Software (Thermo Fisher Scientific, Waltham, MA, USA). A Thermo Hypersil Gold C18 column $\left(100 \mathrm{~mm} \times 2.1 \mathrm{~mm} ; 1.9 \mu \mathrm{m}\right.$ particle size) thermostated at $40^{\circ} \mathrm{C}$ was used for analyte separation. A trap column (Thermo Hypercarb, $20 \mathrm{~mm} \times 2.1 \mathrm{~mm} ; 7 \mu \mathrm{m}$ particle size) was positioned immediately after the aqueous and organic LC mobile phases mixing point but before the injector. The aqueous mobile phase (A) consisted of $0.1 \% \mathrm{HCOOH}$ in HPLC-water $(\mathrm{v} / \mathrm{v})$ and the organic mobile phase $(\mathrm{B})$ of $0.1 \% \mathrm{HCOOH}$ in acetonitrile $(\mathrm{v} / \mathrm{v})$. The injection volume was $10 \mu \mathrm{L}$ or $15 \mu \mathrm{L}$ (for TOP assays).

Chromatographic gradient elution conditions were as follows: gradual increase of $B$ channel from 10 to $72.5 \%(0-7 \mathrm{~min})$, and then from 72.5 to $100 \%$ (7-8.5 $\mathrm{min})$. The $0: 100 \mathrm{~A}$ : B ratio was held for 4 minutes (8.5-12.5 min), then returned to the 90:10 initial set up (12.5-12.6 min), kept constant for 2 minutes for reequilibration (12.6-14.5 min). Before each injection, the injection needle and injection port were rinsed sequentially with i) an equal volumetric mixture of acetonitrile/methanol/isopropanol and ii) HPLC-water containing $0.1 \% \mathrm{HCOOH}$.

Analyte detection was performed using a Q-Exactive Orbitrap mass spectrometer controlled by the Xcalibur 4.0 software (Thermo Fisher Scientific, Waltham, MA, USA) in full scan mode and with $\mathrm{t}-\mathrm{MS}^{2}$ mode, with positive and negative heated electrospray ionization (fast polarity switching mode). ${ }^{3,8}$ Orbitrap parameters were set as follows: AGC target (maximum capacity in C-trap) was set at $3 \times 10^{6}$, maximum injection time at $50 \mathrm{~ms}$, and resolution at 70,000 FWHM at $m / z 200$. The mass scan range was set at $m / z 150-1000$ (Full Scan MS mode). 


\section{Quality assurance/quality control}

Replicate field/trip water blanks were performed (two for each FTA site), and consisted of DI water poured on-site in pre-cleaned HDPE bottles during the sampling campaigns. The blank samples were shipped together with the other field samples and all samples were processed together at the analytical facilities. Method (laboratory) blanks were also performed for both water and soil samples. The method blanks and field/trip blanks presented nondetectable levels of PFAS.

Upon the characterization of low to moderate matrix effects $(< \pm 25 \%$, listed in Table S5), solvent-based calibration curves were used for both soil and groundwater samples. Analytes were quantified using inverse-weighted internal regression lines with determination coefficients $\left(R^{2}\right)$ ranging from 0.9906 to 0.9999 and suitable accuracy (70.6-130.5\%). After running the calibration curve, continued calibration verification standards (quality control CCV samples) were inserted every 10-15 samples during the LC-MS batch sequence. The mean accuracy of CCV standards $(n=5)$ ranged between $80-119 \%$ (Table S10), within the $70-130 \%$ acceptance criterion set by EPA methods. ${ }^{9}$ As an additional control of precision, field duplicates for two soil samples and method triplicates for four groundwater samples were performed. 


\section{Rationales for associating quantification confidence levels}

Quantification confidence levels associated with each of the detected PFASs in the samples from the field survey are shown in Table S9. To attain the highest quantification confidence level, a certified native standard and a matching IS, or at least closely related ones, were used if available. ${ }^{8}$ Identification confidence levels were assigned adapted from Schymanski's classification. ${ }^{10}$ 
Table S1. Ion formula, theoretical and observed $\mathrm{m} / \mathrm{z}$, mass error, retention time (RT) and commercial sources of 53 native PFASs with available standards.

\begin{tabular}{|c|c|c|c|c|c|c|c|}
\hline Analyte & Name & Ion Formula & $\begin{array}{l}\text { Theoretical } \\
\mathrm{m} / \mathbf{z}\end{array}$ & Observed m/z & $\begin{array}{l}\text { Error } \\
(\mathrm{ppm})\end{array}$ & RT (min) & $\begin{array}{l}\text { Sources of } \\
\text { standards }\end{array}$ \\
\hline PFPrA & Perfluoropropionoic acid & {$\left[\mathrm{C}_{3} \mathrm{~F}_{5} \mathrm{O}_{2}\right]^{-}$} & 162.98185 & 162.98169 & -1.0 & 0.89 & \multirow{23}{*}{$\begin{array}{l}\text { PFAC-MXC from } \\
\text { Wellington } \\
\text { Laboratories (Guelph, } \\
\text { ON, Canada) }\end{array}$} \\
\hline PFBA & Perfluorobutanoic acid & {$\left[\mathrm{C}_{4} \mathrm{~F}_{7} \mathrm{O}_{2}\right]^{-}$} & 212.97947 & 212.97906 & -1.9 & 2.21 & \\
\hline PFPeA & Perfluoropentanoic acid & {$\left[\mathrm{C}_{5} \mathrm{~F}_{9} \mathrm{O}_{2}\right]^{-}$} & 262.97669 & 262.97644 & -1.0 & 3.33 & \\
\hline PFHxA & Perfluorohexanoic acid & {$\left[\mathrm{C}_{6} \mathrm{~F}_{11} \mathrm{O}_{2}\right]^{-}$} & 312.97335 & 312.97336 & 0.0 & 4.15 & \\
\hline PFHpA & Perfluoroheptanoic acid & {$\left[\mathrm{C}_{7} \mathrm{~F}_{13} \mathrm{O}_{2}\right]^{-}$} & 362.97013 & 362.97055 & 1.2 & 4.82 & \\
\hline PFOA & Perfluorooctanoic acid & {$\left[\mathrm{C}_{8} \mathrm{~F}_{15} \mathrm{O}_{2}\right]^{-}$} & 412.96714 & 412.96701 & -0.3 & 5.40 & \\
\hline PFNA & Perfluorononanoic acid & {$\left[\mathrm{C}_{9} \mathrm{~F}_{17} \mathrm{O}_{2}\right]^{-}$} & 462.96414 & 462.96408 & -0.1 & 5.96 & \\
\hline PFDA & Perfluorodecanoic acid & {$\left[\mathrm{C}_{10} \mathrm{~F}_{19} \mathrm{O}_{2}\right]^{-}$} & 512.96066 & 512.96063 & -0.1 & 6.50 & \\
\hline PFUnA & Perfluoroundecanoic acid & {$\left[\mathrm{C}_{11} \mathrm{~F}_{21} \mathrm{O}_{2}\right]^{-}$} & 562.95865 & 562.95782 & -1.5 & 7.00 & \\
\hline PFDoA & Perfluorododecanoic acid & {$\left[\mathrm{C}_{12} \mathrm{~F}_{23} \mathrm{O}_{2}\right]^{-}$} & 612.95461 & 612.95477 & 0.3 & 7.52 & \\
\hline PFTrDA & Perfluorotridecanoic acid & {$\left[\mathrm{C}_{13} \mathrm{~F}_{25} \mathrm{O}_{2}\right]^{-}$} & 662.95041 & 662.95209 & 2.5 & 7.99 & \\
\hline PFTeDA & Perfluorotetradecanoic acid & {$\left[\mathrm{C}_{14} \mathrm{~F}_{27} \mathrm{O}_{2}\right]^{-}$} & 712.94808 & 712.94928 & 1.7 & 8.45 & \\
\hline PFHxDA & Perfluorohexadecanoic acid & {$\left[\mathrm{C}_{16} \mathrm{~F}_{31} \mathrm{O}_{2}\right]^{-}$} & 812.94292 & 812.94379 & 1.1 & 9.26 & \\
\hline PFOcDA & Perfluorooctadecanoic acid & {$\left[\mathrm{C}_{18} \mathrm{~F}_{35} \mathrm{O}_{2}\right]-$} & 912.93394 & 912.93701 & 3.4 & 10.06 & \\
\hline PFPrS & Perfluoropropane sulfonate & {$\left[\mathrm{C}_{3} \mathrm{~F}_{7} \mathrm{SO}_{3}\right]^{-}$} & 248.94564 & 248.94719 & 6.2 & 3.19 & \\
\hline PFBS & Perfluorobutane sulfonate & {$\left[\mathrm{C}_{4} \mathrm{~F}_{9} \mathrm{SO}_{3}\right]^{-}$} & 298.94326 & 298.94351 & 0.8 & 4.15 & \\
\hline PFPeS & Perfluorohexane sulfonate & {$\left[\mathrm{C}_{5} \mathrm{~F}_{11} \mathrm{SO}_{3}\right]^{-}$} & 348.93925 & 348.94052 & 3.6 & 4.90 & \\
\hline PFHxS & Perfluorohexane sulfonate & {$\left[\mathrm{C}_{6} \mathrm{~F}_{13} \mathrm{SO}_{3}\right]^{-}$} & 398.93712 & 398.93719 & 0.2 & 5.52 & \\
\hline PFHpS & Perfluoroheptane sulfonate & {$\left[\mathrm{C}_{7} \mathrm{~F}_{15} \mathrm{SO}_{3}\right]^{-}$} & 448.93286 & 448.93408 & 2.7 & 6.08 & \\
\hline PFOS & Perfluorooctane sulfonate & {$\left[\mathrm{C}_{8} \mathrm{~F}_{17} \mathrm{SO}_{3}\right]^{-}$} & 498.93126 & 498.93008 & -2.4 & 6.60 & \\
\hline PFNS & Perfluorononane sulfonate & {$\left[\mathrm{C}_{9} \mathrm{~F}_{19} \mathrm{SO}_{3}\right]^{-}$} & 548.92647 & 548.92798 & 2.8 & 7.12 & \\
\hline PFDS & Perfluorodecane sulfonate & {$\left[\mathrm{C}_{10} \mathrm{~F}_{21} \mathrm{SO}_{3}\right]^{-}$} & 598.92487 & 598.92474 & -0.2 & 7.60 & \\
\hline PFDoS & Perfluorododecane sulfonate & {$\left[\mathrm{C}_{12} \mathrm{~F}_{25} \mathrm{SO}_{3}\right]^{-}$} & 698.91689 & 698.91937 & 3.5 & 8.47 & \\
\hline PFECHS & Perfluoro-4-ethylcyclohexane sulfonate & {$\left[\mathrm{C}_{8} \mathrm{~F}_{15} \mathrm{SO}_{3}\right]^{-}$} & 460.93286 & 460.93585 & 6.5 & 6.02 & \multirow{10}{*}{$\begin{array}{l}\text { Wellington } \\
\text { Laboratories (Guelph, } \\
\text { ON, Canada) }\end{array}$} \\
\hline FBSA & Perfluorobutane sulfonamide & {$\left[\mathrm{C}_{4} \mathrm{~F}_{9} \mathrm{SO}_{2} \mathrm{NH}\right]^{-}$} & 297.95843 & 297.95938 & 3.2 & 5.21 & \\
\hline FHxSA & Perfluorohexane sulfonamide & {$\left[\mathrm{C}_{6} \mathrm{~F}_{13} \mathrm{SO}_{2} \mathrm{NH}\right]^{-}$} & 397.95204 & 397.95282 & 2.0 & 6.56 & \\
\hline FOSA & Perfluorooctane sulfonamide & {$\left[\mathrm{C}_{8} \mathrm{~F}_{17} \mathrm{SO}_{2} \mathrm{NH}\right]^{-}$} & 497.94631 & 497.94745 & 2.3 & 7.58 & \\
\hline MeFOSA & $\mathrm{N}$-methyl-perfluorooctane sulfonamide & {$\left[\mathrm{C}_{9} \mathrm{~F}_{17} \mathrm{SO}_{2} \mathrm{NH}_{3}\right]^{-}$} & 511.96130 & 511.96326 & 3.8 & 8.18 & \\
\hline EtFOSA & $\mathrm{N}$-ethyl-perfluorooctane sulfonamide & {$\left[\mathrm{C}_{10} \mathrm{~F}_{17} \mathrm{SO}_{2} \mathrm{NH}_{5}\right]^{-}$} & 525.97695 & 525.97882 & 3.6 & 8.41 & \\
\hline FOSAA & Perfluorooctane sulfonamidoacetic acid & {$\left[\mathrm{C}_{10} \mathrm{~F}_{17} \mathrm{SO}_{4} \mathrm{NH}_{3}\right]^{-}$} & 555.95113 & 555.95331 & 3.9 & 7.48 & \\
\hline MeFOSAA & $\mathrm{N}$-methyl-perfluorooctane sulfonamidoacetic acid & {$\left[\mathrm{C}_{11} \mathrm{~F}_{17} \mathrm{SO}_{4} \mathrm{NH}_{5}\right]^{-}$} & 569.96678 & 569.96893 & 3.8 & 7.51 & \\
\hline EtFOSAA & $\mathrm{N}$-ethyl-perfluorooctane sulfonamidoacetic acid & {$\left[\mathrm{C}_{12} \mathrm{~F}_{17} \mathrm{SO}_{4} \mathrm{NH}_{7}\right]^{-}$} & 583.98243 & 583.98511 & 4.6 & 8.20 & \\
\hline 3:3 FTCA & 3:3 fluorotelomer carboxylate & {$\left[\mathrm{C}_{6} \mathrm{~F}_{7} \mathrm{H}_{4} \mathrm{O}_{2}\right]^{-}$} & 241.00995 & 241.01096 & 4.2 & 4.94 & \\
\hline 4:3 FTCA & $4: 3$ fluorotelomer carboxylate & {$\left[\mathrm{C}_{7} \mathrm{~F}_{9} \mathrm{H}_{4} \mathrm{O}_{2}\right]^{-}$} & 291.00676 & 291.00836 & 5.5 & 5.69 & $\begin{array}{l}\text { DuPont (Wilmington, } \\
\text { DE, USA) }\end{array}$ \\
\hline
\end{tabular}




\begin{tabular}{|c|c|c|c|c|c|c|c|}
\hline 5:3 FTCA & $5: 3$ fluorotelomer carboxylate & {$\left[\mathrm{C}_{8} \mathrm{~F}_{11} \mathrm{H}_{4} \mathrm{O}_{2}\right]^{-}$} & 341.00356 & 341.00516 & 4.7 & 6.31 & \multirow{12}{*}{$\begin{array}{l}\text { Wellington } \\
\text { Laboratories (Guelph, } \\
\text { ON, Canada) }\end{array}$} \\
\hline 7:3 FTCA & $7: 3$ fluorotelomer carboxylate & {$\left[\mathrm{C}_{10} \mathrm{~F}_{15} \mathrm{H}_{4} \mathrm{O}_{2}\right]^{-}$} & 440.99717 & 440.99921 & 4.6 & 7.39 & \\
\hline 4:2 FTSA & $4: 2$ fluorotelomer sulfonate & {$\left[\mathrm{C}_{6} \mathrm{~F}_{9} \mathrm{H}_{4} \mathrm{SO}_{2}\right]^{-}$} & 326.97374 & 326.97528 & 4.7 & 3.86 & \\
\hline 6:2 FTSA & 6:2 fluorotelomer sulfonate & {$\left[\mathrm{C}_{8} \mathrm{~F}_{13} \mathrm{H}_{4} \mathrm{SO}_{2}\right]^{-}$} & 426.96866 & 426.96902 & 0.8 & 5.11 & \\
\hline 8:2 FTSA & $8: 2$ fluorotelomer sulfonate & {$\left[\mathrm{C}_{10} \mathrm{~F}_{17} \mathrm{H}_{4} \mathrm{SO}_{2}\right]^{-}$} & 526.96097 & 526.96289 & 3.6 & 6.17 & \\
\hline 10:2 FTSA & 10:2 fluorotelomer sulfonate & {$\left[\mathrm{C}_{12} \mathrm{~F}_{21} \mathrm{H}_{4} \mathrm{SO}_{3}\right]^{-}$} & 626.95458 & 626.95715 & 4.1 & 7.18 & \\
\hline 6:2 FTUA & $6: 2$ fluorotelomer unsaturated acid & {$\left[\mathrm{C}_{8} \mathrm{~F}_{12} \mathrm{H}_{2} \mathrm{O}_{2}\right]^{-}$} & 356.97849 & 356.98026 & 5.0 & 6.46 & \\
\hline 8:2 FTUA & $8: 2$ fluorotelomer unsaturated acid & {$\left[\mathrm{C}_{10} \mathrm{~F}_{16} \mathrm{H}_{2} \mathrm{O}_{2}\right]^{-}$} & 456.97210 & 456.97412 & 4.4 & 7.54 & \\
\hline 10:2 FTUA & 10:2 fluorotelomer unsaturated acid & {$\left[\mathrm{C}_{12} \mathrm{~F}_{20} \mathrm{H}_{2} \mathrm{O}_{2}\right]^{-}$} & 556.96571 & 556.96808 & 4.3 & 8.39 & \\
\hline 6:2 FTAB & 6:2 fluorotelomer sulfonamidoalkyl betaine & {$\left[\mathrm{C}_{15} \mathrm{~F}_{13} \mathrm{H}_{20} \mathrm{~N}_{2} \mathrm{SO}_{4}\right]^{+}$} & 571.09362 & 571.09387 & 0.4 & 5.37 & \\
\hline 5:3 FTB & $5: 3$ fluorotelomer betaine & {$\left[\mathrm{C}_{12} \mathrm{~F}_{11} \mathrm{H}_{15} \mathrm{NO}_{2}\right]^{+}$} & 414.09271 & 414.09247 & -0.6 & 4.47 & \\
\hline $5: 1: 2$ FTB & $5: 1: 2$ fluorotelomer betaine & {$\left[\mathrm{C}_{12} \mathrm{~F}_{12} \mathrm{H}_{14} \mathrm{NO}_{2}\right]^{+}$} & 432.08329 & 432.08324 & -0.1 & 4.58 & \\
\hline $\begin{array}{l}\text { PFHxSAm(AmPr- } \\
\text { FHxSA) }\end{array}$ & Perfluorohexane sulfonamidoalkyl amine & {$\left[\mathrm{C}_{11} \mathrm{H}_{14} \mathrm{~F}_{13} \mathrm{~N}_{2} \mathrm{O}_{2} \mathrm{~S}\right]^{+}$} & 485.05684 & 485.05692 & 0.2 & 5.28 & \multirow{8}{*}{$\begin{array}{l}\text { Beijing Surfactant } \\
\text { Institute (Peking, } \\
\text { China). }\end{array}$} \\
\hline $\begin{array}{l}\text { PFOSAm(AmPr- } \\
\text { FOSA) }\end{array}$ & Perfluorooctane sulfonamidoalkyl amine & {$\left[\mathrm{C}_{13} \mathrm{H}_{14} \mathrm{~F}_{17} \mathrm{~N}_{2} \mathrm{O}_{2} \mathrm{~S}\right]^{+}$} & 585.04990 & 585.05054 & 1.1 & 6.32 & \\
\hline $\begin{array}{l}\text { PFHxSAmS(N- } \\
\text { TAmP-FHxSA) }\end{array}$ & Perfluorohexane sulfonamidoalkyl ammonium & {$\left[\mathrm{C}_{12} \mathrm{H}_{16} \mathrm{~F}_{13} \mathrm{~N}_{2} \mathrm{O}_{2} \mathrm{~S}\right]^{+}$} & 499.07249 & 499.07251 & 0.0 & 5.34 & \\
\hline $\begin{array}{l}\text { PFOSAmS(N- } \\
\text { TAmP-FOSA) }\end{array}$ & Perfluorooctane sulfonamidoalkyl ammonium & {$\left[\mathrm{C}_{14} \mathrm{H}_{16} \mathrm{~F}_{17} \mathrm{~N}_{2} \mathrm{O}_{2} \mathrm{~S}\right]^{+}$} & 599.06555 & 599.06610 & 0.9 & 6.38 & \\
\hline $\begin{array}{l}\text { PFOSNO(N- } \\
\text { OxAmP-FOSA) }\end{array}$ & Perfluorooctane sulfonamidoalkyl amine oxide & {$\left[\mathrm{C}_{13} \mathrm{H}_{14} \mathrm{~F}_{17} \mathrm{~N}_{2} \mathrm{O}_{3} \mathrm{~S}\right]^{+}$} & 601.04482 & 601.04510 & 0.5 & 6.36 & \\
\hline $\begin{array}{l}\text { PFOANO(N- } \\
\text { OxAmP-FOAd) }\end{array}$ & Perfluorooctane amidoalkyl amine oxide & {$\left[\mathrm{C}_{13} \mathrm{H}_{14} \mathrm{~F}_{15} \mathrm{~N}_{2} \mathrm{O}_{2}\right]^{+}$} & 515.08103 & 515.08197 & 1.8 & 5.49 & \\
\hline $\begin{array}{l}\text { PFOSB(N- } \\
\text { CMAmP-FOSA) }\end{array}$ & Perfluorooctane sulfonamidoalkyl betaine & {$\left[\mathrm{C}_{15} \mathrm{H}_{16} \mathrm{~F}_{17} \mathrm{~N}_{2} \mathrm{O}_{4} \mathrm{~S}\right]^{+}$} & 643.05538 & 643.05591 & 0.8 & 6.32 & \\
\hline $\begin{array}{l}\mathrm{PFOAB}(\mathrm{N}- \\
\mathrm{CMAmP-FOAd})\end{array}$ & Perfluorooctane amidoalkyl betaine & {$\left[\mathrm{C}_{15} \mathrm{H}_{16} \mathrm{~F}_{15} \mathrm{~N}_{2} \mathrm{O}_{3}\right]^{+}$} & 557.09159 & 557.09229 & 1.3 & 5.39 & \\
\hline
\end{tabular}


Table S2. The acronym, full name, theoretical and observed $\mathrm{m} / \mathrm{z}, \mathrm{RT}$ and commercial sources of isotope-labeled IS.

(a) Acronym, full name, theoretical and observed $\mathrm{m} / \mathrm{z}, \mathrm{RT}$ and commercial sources of surrogate IS.

\begin{tabular}{|c|c|c|c|c|c|c|c|c|c|}
\hline Acronym & Acronym & Full Name & $\begin{array}{l}\mathrm{M}^{+},[\mathrm{M}+\mathrm{H}]^{+} \text {or }[\mathrm{M}- \\
\mathrm{H}]^{-}\end{array}$ & $\begin{array}{l}\text { Theoretical } \\
\mathrm{m} / \mathbf{z}\end{array}$ & $\begin{array}{l}\text { Observed } \\
\mathrm{m} / \mathbf{z}\end{array}$ & $\begin{array}{l}\text { Mass } \\
\text { Error, ppm }\end{array}$ & RT, min & $\begin{array}{l}\text { lonization } \\
\text { mode }\end{array}$ & $\begin{array}{l}\text { Commercial } \\
\text { Sources }\end{array}$ \\
\hline${ }^{13} \mathrm{C}_{4}$-PFBA & MPFBA & $\begin{array}{l}\text { Perfluoro-n-[1,2,3,4- } \\
{ }^{13} \mathrm{C}_{\Delta} \text { lbutanoic acid }\end{array}$ & {$\left[{ }^{13} \mathrm{C}_{4} \mathrm{~F}_{7} \mathrm{O}_{2}\right]^{-}$} & 216.99177 & 216.99271 & 4.3 & 2.21 & ESI- & \multirow{22}{*}{$\begin{array}{l}\text { Wellington } \\
\text { Laboratories } \\
\text { (Guelph, } \\
\text { ON, } \\
\text { Canada) }\end{array}$} \\
\hline${ }^{13} \mathrm{C}_{5}-\mathrm{PFPeA}$ & M5PFPeA & Perfluoro-n- $\left[{ }^{13} \mathrm{C}_{5}\right]$ pentanoic acid & {$\left[{ }^{13} \mathrm{C}_{5} \mathrm{~F}_{9} \mathrm{O}_{2}\right]^{-}$} & 267.99345 & 267.99338 & -0.3 & 3.33 & ESI- & \\
\hline${ }^{13} \mathrm{C}_{5}-\mathrm{PFH} \times \mathrm{A}$ & M5PFHxA & $\begin{array}{l}\text { Perfluoro-n-[1,2,3,4,6- } \\
{ }^{13} \mathrm{C}_{5} \text { lhexanoic acid }\end{array}$ & {$\left[{ }^{13} \mathrm{C}_{5} \mathrm{CF}_{11} \mathrm{O}_{2}\right]^{-}$} & 317.99046 & 317.99026 & -0.6 & 4.15 & ESI- & \\
\hline${ }^{13} \mathrm{C}_{4}-\mathrm{PFHpA}$ & M4PFHpA & $\begin{array}{l}\text { Perfluoro-n-[1,2,3,4- } \\
{ }^{13} \mathrm{C}_{4} \text { ]heptanoic acid }\end{array}$ & {$\left[{ }^{13} \mathrm{C}_{4} \mathrm{C}_{3} \mathrm{~F}_{13} \mathrm{O}_{2}\right]^{-}$} & 366.98249 & 366.98407 & 4.3 & 4.82 & ESI- & \\
\hline${ }^{13} \mathrm{C}_{8}$-PFOA & M8PFOA & Perfluoro-n- $\left[{ }^{13} \mathrm{C}_{8}\right]$ octanoic acid & {$\left[{ }^{13} \mathrm{C}_{8} \mathrm{~F}_{15} \mathrm{O}_{2}\right]^{-}$} & 420.99272 & 420.99429 & 3.7 & 5.40 & ESI- & \\
\hline${ }^{13} \mathrm{C}_{9}$-PFNA & M9PFNA & Perfluoro-n- $\left[{ }^{13} \mathrm{C}_{9}\right]$ nonanoic acid & {$\left[{ }^{13} \mathrm{C}_{9} \mathrm{~F}_{17} \mathrm{O}_{2}\right]^{-}$} & 471.99288 & 471.99435 & 3.1 & 5.96 & ESI- & \\
\hline${ }^{13} \mathrm{C}_{6}-\mathrm{PFDA}$ & M6PFDA & $\begin{array}{l}\text { Perfluoro-n-[1,2,3,4,5,6- } \\
{ }^{13} \mathrm{C}_{6} \text { ldecanoic acid }\end{array}$ & {$\left[{ }^{13} \mathrm{C}_{6} \mathrm{C}_{4} \mathrm{~F}_{19} \mathrm{O}_{2}\right]^{-}$} & 518.97962 & 518.98120 & 3.0 & 6.50 & ESI- & \\
\hline${ }^{13} C_{7}$-PFUnA & M7PFUdA & $\begin{array}{l}\text { Perfluoro-n-[1,2,3,4,5,6,7- } \\
{ }^{13} \mathrm{C}_{7} \text { lundecanoic acid }\end{array}$ & {$\left[{ }^{13} \mathrm{C}_{7} \mathrm{C}_{4} \mathrm{~F}_{21} \mathrm{O}_{2}\right]^{-}$} & 569.97978 & 569.98175 & 3.5 & 7.00 & ESI- & \\
\hline${ }^{13} \mathrm{C}_{2}$-PFDoA & MPFDoA & $\begin{array}{l}\text { Perfluoro-n-[1,2- } \\
{ }^{13} \mathrm{C}_{2} \text { ldodecanoic acid }\end{array}$ & {$\left[{ }^{13} \mathrm{C}_{2} \mathrm{C}_{10} \mathrm{~F}_{23} \mathrm{O}_{2}\right]^{-}$} & 614.95981 & 614.96191 & 3.4 & 7.52 & ESI- & \\
\hline${ }^{13} \mathrm{C}_{2}-\mathrm{PFTeDA}$ & M2PFTeDA & $\begin{array}{l}\text { Perfluoro-n-[1,2- } \\
\left.{ }^{13} \mathrm{C}_{2}\right] \text { tetradecanoic acid }\end{array}$ & {$\left[{ }^{13} \mathrm{C}_{2} \mathrm{C}_{12} \mathrm{~F}_{27} \mathrm{O}_{2}\right]^{-}$} & 714.95342 & 714.95636 & 4.1 & 8.45 & ESI- & \\
\hline${ }^{13} \mathrm{C}_{3}$-PFBS & M3PFBS & $\begin{array}{l}\text { Perfluoro-1-[2,3,4- } \\
{ }^{13} \mathrm{C}_{3} \text { lbutanesulfonate }\end{array}$ & {$\left[{ }^{13} \mathrm{C}_{3} \mathrm{C}_{1} \mathrm{~F}_{9} \mathrm{SO}_{3}\right]^{-}$} & 301.95251 & 301.95352 & 3.3 & 4.15 & ESI- & \\
\hline${ }^{13} \mathrm{C}_{3}$-PFHXS & M3PFHxS & $\begin{array}{l}\text { Perfluoro- } 1-[1,2,3- \\
{ }^{13} \mathrm{C}_{3} \text { lhexanesulfonate }\end{array}$ & {$\left[{ }^{13} \mathrm{C}_{3} \mathrm{C}_{3} \mathrm{~F}_{13} \mathrm{SO}_{3}\right]^{-}$} & 401.94612 & 401.94727 & 2.9 & 5.52 & ESI- & \\
\hline${ }^{13} \mathrm{C}_{8}$-PFOS & M8PFOS & $\begin{array}{l}\text { Perfluoro-1- } \\
{ }^{13} \mathrm{C}_{8} \text { loctanesulfonate }\end{array}$ & {$\left[{ }^{13} \mathrm{C}_{8} \mathrm{~F}_{17} \mathrm{SO}_{3}\right]^{-}$} & 506.95641 & 506.95837 & 3.9 & 6.60 & ESI- & \\
\hline${ }^{13} \mathrm{C}_{2}-6: 2 \mathrm{FtS}$ & $\begin{array}{l}\text { M2-6:2 } \\
\text { FTSA }\end{array}$ & $\begin{array}{l}1 \mathrm{H}, 1 \mathrm{H}, 2 \mathrm{H}, 2 \mathrm{H} \text {-perfluoro-1-[1,2- } \\
{ }^{13} \mathrm{C}_{2} \mathrm{l}-\mathrm{octane} \text { sulfonate }\end{array}$ & {$\left[{ }^{13} \mathrm{C}_{2} \mathrm{C}_{8} \mathrm{~F}_{16} \mathrm{HO}_{2}\right]^{-}$} & 428.97537 & 428.97568 & 0.7 & 5.11 & ESI- & \\
\hline${ }^{13} \mathrm{C}_{2}-8: 2 \mathrm{FtS}$ & M2-8:2FTSA & $\begin{array}{l}1 \mathrm{H}, 1 \mathrm{H}, 2 \mathrm{H}, 2 \mathrm{H} \text {-perfluoro-1-[1,2- } \\
{ }^{13} \mathrm{C}_{2} \text {-decane sulfonate }\end{array}$ & {$\left[\mathrm{C}_{10} \mathrm{~F}_{17} \mathrm{D}_{5} \mathrm{NSO}_{2}\right]^{-}$} & 528.96898 & 528.96960 & 1.2 & 6.17 & ESI- & \\
\hline${ }^{13} \mathrm{C}_{2}-6: 2$ FTUA & M6:2 FTUA & $\begin{array}{l}\left.\text { 2H-Perfluoro-[1,2- }{ }^{13} \mathrm{C}_{2}\right]-2- \\
\text { octenoic acid }\end{array}$ & {$\left[{ }^{13} \mathrm{C}_{2} \mathrm{C}_{8} \mathrm{~F}_{17} \mathrm{H}_{4} \mathrm{SO}_{3}\right]^{-}$} & 358.98520 & 358.98685 & 4.6 & 6.46 & ESI- & \\
\hline${ }^{13} \mathrm{C}_{2}-8: 2$ FTUA & M8:2 FTUA & $\begin{array}{l}\left.\text { 2H-Perfluoro-[1,2- }{ }^{13} \mathrm{C}_{2}\right]-2- \\
\text { decenoic acid }\end{array}$ & {$\left[{ }^{13} \mathrm{C}_{2} \mathrm{C}_{6} \mathrm{~F}_{12} \mathrm{HO}_{2}\right]^{-}$} & 458.97881 & 458.98083 & 4.4 & 7.54 & ESI- & \\
\hline${ }^{13} \mathrm{C}_{8}-\mathrm{FOSA}$ & M8FOSA-I & $\begin{array}{l}\text { Perfluoro-1- }-\left[{ }^{13} \mathrm{C}_{8}\right] \text { octane } \\
\text { sulfonamide }\end{array}$ & {$\left[{ }^{13} \mathrm{C}_{8} \mathrm{~F}_{17} \mathrm{NHSO}_{2}\right]^{-}$} & 505.97249 & 505.97430 & 3.6 & 7.58 & ESI- & \\
\hline$d_{3}-N-M e F O S A$ & \multirow{4}{*}{$\begin{array}{l}\text { d-N- } \\
\text { MeFOSA-M } \\
\text { d-N- } \\
\text { EtFOSA-M } \\
\text { d3-N- } \\
\text { MeFOSAA } \\
\text { d5-N- } \\
\text { EtFOSAA }\end{array}$} & $\begin{array}{l}\mathrm{N} \text {-methyl- } \mathrm{d}_{3} \text {-perfluoro-1- } \\
\text { octanesulfonamide }\end{array}$ & {$\left[\mathrm{C}_{9} \mathrm{~F}_{17} \mathrm{NSO}_{2} \mathrm{D}_{3}\right]_{-}$} & 514.98013 & 514.98187 & 3.4 & 8.18 & ESI- & \\
\hline$d_{5}-N-E t F O S A$ & & $\begin{array}{l}\text { N-ethyl-d5-perfluoro-1- } \\
\text { octanesulfonamide }\end{array}$ & {$\left[\mathrm{C}_{10} \mathrm{~F}_{17} \mathrm{NSO}_{2} \mathrm{D}_{5}\right]^{-}$} & 531.00830 & 531.01001 & 3.2 & 8.41 & ESI- & \\
\hline $\begin{array}{l}d_{3}-N- \\
\text { MeFOSAA }\end{array}$ & & $\begin{array}{l}\mathrm{N} \text {-methyl- } \mathrm{d}_{3} \text {-perfluoro-1- } \\
\text { octanesulfonamidoacetic acid }\end{array}$ & {$\left[\mathrm{C}_{11} \mathrm{~F}_{17} \mathrm{NSO}_{4} \mathrm{D}_{3} \mathrm{H}_{2}\right]^{-}$} & 572.98561 & 572.98798 & 4.1 & 7.94 & ESI- & \\
\hline$d_{5}-N-E t F O S A A$ & & $\begin{array}{l}\mathrm{N} \text {-ethyl-d5-perfluoro-1- } \\
\text { octanesulfonamidoacetic acid }\end{array}$ & {$\left[\mathrm{C}_{12} \mathrm{~F}_{17} \mathrm{NSO}_{4} \mathrm{D}_{5} \mathrm{H}_{2}\right]^{-}$} & 589.01437 & 589.01599 & 2.8 & 8.20 & ESI- & \\
\hline
\end{tabular}




\begin{tabular}{|c|c|c|c|c|c|c|c|c|c|}
\hline $\begin{array}{l}\text { PFOAAmS(N- } \\
\text { TAmP-FOAd) }\end{array}$ & PFOAAmS & $\begin{array}{l}\text { Perfluorooctane } \\
\text { ammonium }\end{array}$ & {$\left[\mathrm{C}_{14} \mathrm{H}_{16} \mathrm{~F}_{15} \mathrm{~N}_{2} \mathrm{O}\right]^{+}$} & 513.10176 & 513.10254 & 1.5 & 5.38 & ESI+ & $\begin{array}{l}\text { Beijing } \\
\text { Surfactant } \\
\text { Institute } \\
\text { (Peking, } \\
\end{array}$ \\
\hline Acronym & Acronym & Full Name & $\begin{array}{l}\mathrm{M}^{+},[\mathrm{M}+\mathrm{H}]^{+} \text {or }[\mathrm{M}- \\
\mathrm{H}]^{-}\end{array}$ & $\begin{array}{l}\text { Theoretical } \\
\mathrm{m} / \mathbf{z}\end{array}$ & $\begin{array}{l}\text { Observed } \\
\mathrm{m} / \mathrm{z}\end{array}$ & $\begin{array}{l}\text { Mass } \\
\text { Error, ppm }\end{array}$ & $\mathbf{R T}, \min$ & $\begin{array}{l}\text { lonization } \\
\text { mode }\end{array}$ & $\begin{array}{l}\text { Commercial } \\
\text { Sources }\end{array}$ \\
\hline${ }^{13} \mathrm{C}_{3}$-PFBA & M3PFBA & $\begin{array}{l}\text { Perfluoro-n-[2,3,4-13C3]butanoic } \\
\text { acid }\end{array}$ & {$\left[{ }^{13} \mathrm{C}_{3} \mathrm{CF}_{7} \mathrm{O}_{2}\right]^{-}$} & 215.98926 & 215.98917 & -0.4 & 2.21 & ESI- & \multirow{4}{*}{$\begin{array}{l}\text { Wellington } \\
\text { Laboratories } \\
\text { (Guelph, } \\
\text { ON, } \\
\text { Canada) }\end{array}$} \\
\hline${ }^{13} \mathrm{C}_{2}$-PFOA & M2PFOA & Perfluoro-n-[1,2-13C2]octanoic acid & {$\left[{ }^{13} \mathrm{C}_{2} \mathrm{C}_{6} \mathrm{~F}_{15} \mathrm{O}_{2}\right]^{-}$} & 414.97314 & 414.97385 & 1.7 & 5.40 & ESI- & \\
\hline${ }^{13} \mathrm{C}_{2}$-PFDA & MPFDA & $\begin{array}{l}\text { Perfluoro-n-[1,2-13C2]decanoic } \\
\text { acid }\end{array}$ & {$\left[{ }^{13} \mathrm{C}_{2} \mathrm{C}_{8} \mathrm{~F}_{19} \mathrm{O}_{2}\right]^{-}$} & 514.96675 & 514.96729 & 1.0 & 6.50 & ESI- & \\
\hline${ }^{13} \mathrm{C}_{4}$-PFOS & MPFOS & $\begin{array}{l}\text { Sodium perfluoro-1-[1,2,3,4- } \\
\text { 13C4]octanesulfonate }\end{array}$ & {$\left[{ }^{13} \mathrm{C}_{4} \mathrm{C}_{4} \mathrm{~F}_{17} \mathrm{SO}_{3}\right]^{-}$} & 502.94364 & 502.94379 & 0.3 & 6.60 & ESI- & \\
\hline
\end{tabular}


Table S3. Detailed information about field soil and groundwater samples.

(a) Soil sample list.

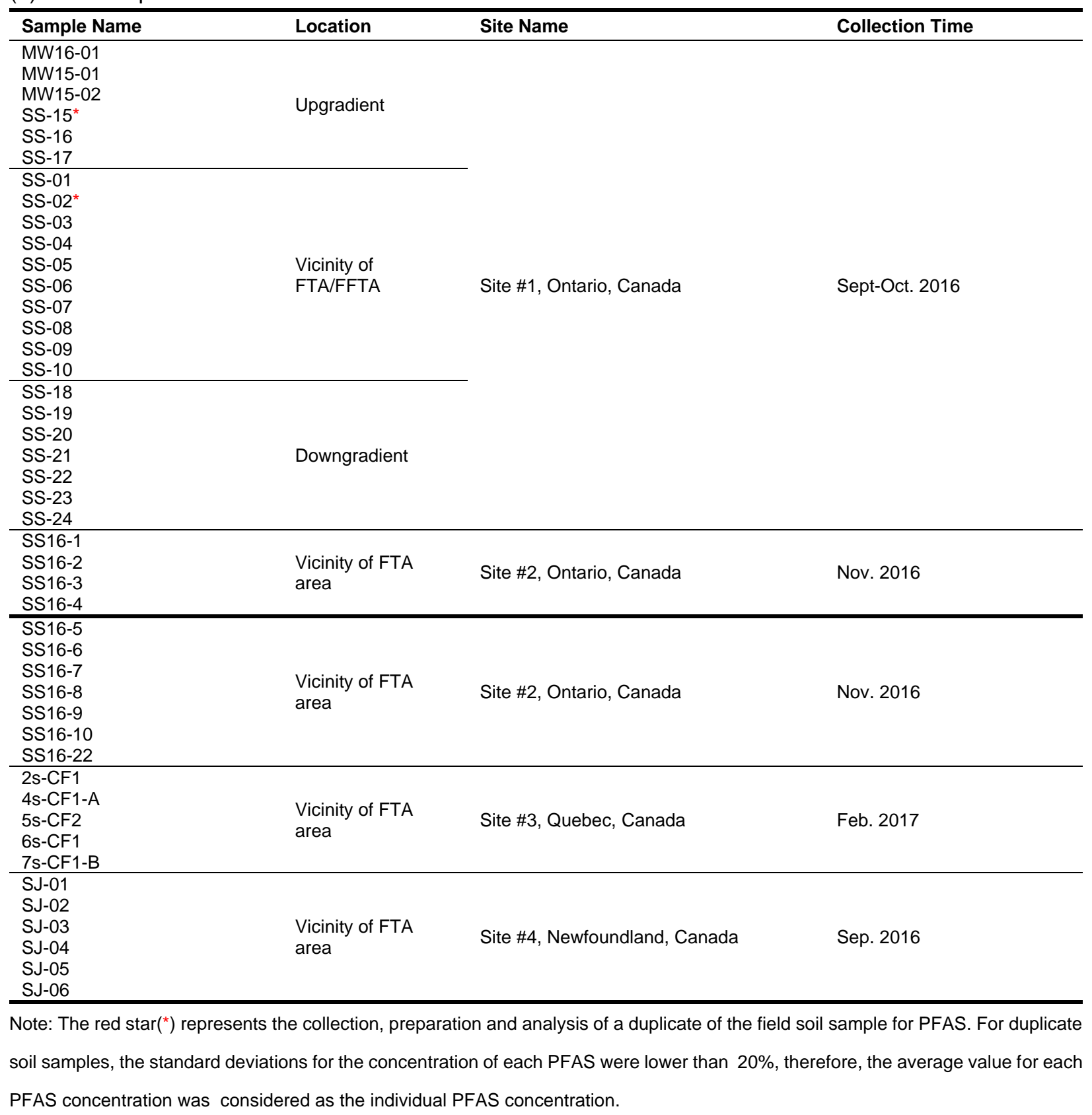


(b) Groundwater sample list.

\begin{tabular}{|c|c|c|c|c|}
\hline Sample Name & $\begin{array}{l}\text { The depths to } \\
\text { groundwater table } \\
\text { (m bgs) }\end{array}$ & Location & Site Name & Collection Time \\
\hline MW16-01 & 2.58 & & \multirow{14}{*}{ Site \#1, Ontario, Canada } & \multirow{14}{*}{ Sept-Oct. 2016} \\
\hline MW15-01 & 2.54 & Upgradient & & \\
\hline MW15-02 & 3.18 & & & \\
\hline 07MW02S & 3.37 & \multirow{5}{*}{$\begin{array}{l}\text { Vicinity of FTA/FFTA } \\
\text { area }\end{array}$} & & \\
\hline TCF & 3.23 & & & \\
\hline P5 & 3.29 & & & \\
\hline MW 0902B & 2.88 & & & \\
\hline MW13-13 & 3.20 & & & \\
\hline $11-01 \mathrm{D}$ & 8.68 & \multirow{6}{*}{ Downgradient } & & \\
\hline $11-02 D$ & 8.89 & & & \\
\hline $11-06 S$ & 1.88 & & & \\
\hline MW13-24D & 3.63 & & & \\
\hline $11-04$ & 3.55 & & & \\
\hline $11-05$ & 4.51 & & & \\
\hline MW14-2 & 2.38 & \multirow{3}{*}{ Upgradient } & \multirow{21}{*}{ Site \#2, Ontario, Canada } & \multirow{21}{*}{ Sept-Oct. 2016} \\
\hline MW12-2B & 2.06 & & & \\
\hline MW 12-4 & 1.76 & & & \\
\hline MW105 & 1.90 & \multirow{12}{*}{ Vicinity of FTA area } & & \\
\hline MW108 & 1.84 & & & \\
\hline L14 & 1.76 & & & \\
\hline MW101 & 2.28 & & & \\
\hline MW110 & 2.29 & & & \\
\hline MW212 & 1.12 & & & \\
\hline MW213 & 1.18 & & & \\
\hline MW215 & 2.07 & & & \\
\hline MW216 & 1.30 & & & \\
\hline MW207 & 1.68 & & & \\
\hline MW208 & 1.13 & & & \\
\hline MW210 & 1.42 & & & \\
\hline MW209 & 1.36 & \multirow{6}{*}{ Downgradient } & & \\
\hline MW15-1 & $\mathrm{NA}^{*}$ & & & \\
\hline MW15-2 & $\mathrm{NA}^{*}$ & & & \\
\hline MW304 & 1.67 & & & \\
\hline MW14-6 & 1.39 & & & \\
\hline MW12-19 & 0.78 & & & \\
\hline PO $158 \mathrm{R}$ & 1.71 & \multirow{18}{*}{ Vicinity of FTA area } & \multirow{18}{*}{ Site \#3, Quebec, Canada } & \multirow{18}{*}{ Sept-Oct. 2016} \\
\hline PO $158 S$ & 1.65 & & & \\
\hline $17 \mathrm{PO} 1 \mathrm{R}$ & 0.48 & & & \\
\hline $17 \mathrm{PO} 1 \mathrm{~S}$ & 0.53 & & & \\
\hline $17 \mathrm{PO} 2 \mathrm{R}$ & 1.24 & & & \\
\hline $17 \mathrm{PO} 2 \mathrm{~S}$ & 1.28 & & & \\
\hline $17 \mathrm{PO} 4 \mathrm{R}$ & 0.62 & & & \\
\hline $17 \mathrm{PO} 4 \mathrm{~S}$ & 0.65 & & & \\
\hline $17 \mathrm{PO} 5 \mathrm{R}$ & 0.86 & & & \\
\hline 17 PO 5S & 0.83 & & & \\
\hline $17 \mathrm{PO} 6 \mathrm{R}$ & 1.16 & & & \\
\hline $17 \mathrm{PO} 6 \mathrm{~S}$ & 1.13 & & & \\
\hline $17 \mathrm{PO} 7 \mathrm{R}$ & 0.58 & & & \\
\hline $17 \mathrm{PO} 7 \mathrm{~S}$ & 0.59 & & & \\
\hline M269 & 0.36 & & & \\
\hline M412R & 0.44 & & & \\
\hline PO $156 R$ & 0.54 & & & \\
\hline PO $156 \mathrm{~S}$ & 0.49 & & & \\
\hline MW16 & $\mathrm{NA}^{*}$ & Upgradient & \multirow{9}{*}{ Site \#4, Newfoundland, Canada } & \multirow{9}{*}{ Sept-Oct. 2016} \\
\hline FTA MW8 & $\mathrm{NA}^{*}$ & \multirow{5}{*}{ Vicinity of FTA area } & & \\
\hline MW 0502 & $N A^{*}$ & & & \\
\hline MW06 & $N A^{*}$ & & & \\
\hline Well 57 & $N A^{*}$ & & & \\
\hline Well 662 & $\mathrm{NA}^{*}$ & & & \\
\hline JW0606 & $\mathrm{NA}^{*}$ & & & \\
\hline JW 06-10 & $N A^{*}$ & Downgradient & & \\
\hline DS2MW8 & $N A^{*}$ & & & \\
\hline
\end{tabular}


Table S4. The property and PFAS background levels of soils used for spike recovery test, matrix effect assessment, and TOP method validation purposes.

(a) Soil property.

\begin{tabular}{lllllllll}
\hline Name & Type & Sampling location & \% sand & \% silt & \% clay & Textural class & $\%$ OM & pH \\
\hline Soil \#1R & Background & $\begin{array}{l}\text { Chaudière watershed, } \\
\text { QC, Canada }\end{array}$ & 59.2 & 32.2 & 8.6 & Sandy loam & 3.1 & 5 \\
Soil \#2N & Background & $\begin{array}{l}\text { Nuns' Island, QC, } \\
\text { Canada }\end{array}$ & 51.2 & 36.2 & 12.6 & Loam & 12.6 & 4.5 \\
Soil \#3F & Background & $\begin{array}{l}\text { Parc Elgar, QC, } \\
\text { Canada }\end{array}$ & 47.2 & 40.0 & 12.8 & Loam & 4.0 & 5.2 \\
\hline
\end{tabular}

(b) The PFAS background levels. Each soil matrix was prepared and analyzed in triplicate using the same extraction (MeOH with $100 \mathrm{mM}$ ammonium acetate) and cleanup method described for field soil samples.

\begin{tabular}{|c|c|c|c|}
\hline PFAS analyte & $\begin{array}{l}\text { Soil from Riverine Chaudière } \\
\text { (1R soil, } \mu \mathrm{g} / \mathrm{kg} \mathrm{dw})\end{array}$ & $\begin{array}{l}\text { Soil from Nun Island } \\
(2 \mathrm{~N} \text { soil, } \mu \mathrm{g} / \mathrm{kg} \mathrm{dw})\end{array}$ & $\begin{array}{l}\text { Soil from Parc Elgar } \\
\text { (3F soil, } \mu \mathrm{g} / \mathrm{kg} \mathrm{dw} \text { ) }\end{array}$ \\
\hline PFPrA & ND & ND & $0.80 \pm 0.10$ \\
\hline PFBA & $0.20 \pm 0.08$ & $1.43 \pm 0.33$ & $0.71 \pm 0.22$ \\
\hline PFPeA & $0.26 \pm 0.01$ & $0.29 \pm 0.01$ & $0.26 \pm 0.05$ \\
\hline PFHxA & $0.11 \pm 0.06$ & $0.37 \pm 0.02$ & $0.27 \pm 0.01$ \\
\hline PFHPA & $0.17 \pm 0.01$ & $0.31 \pm 0.06$ & $0.18 \pm 0.01$ \\
\hline PFOA & $0.16 \pm 0.01$ & $0.43 \pm 0.00$ & $0.38 \pm 0.04$ \\
\hline PFNA & $0.14 \pm 0.03$ & $0.15 \pm 0.02$ & $0.15 \pm 0.01$ \\
\hline PFDA & $0.08 \pm 0.02$ & $0.05 \pm 0.07$ & $0.04 \pm 0.06$ \\
\hline PFUnA & $0.11 \pm 0.01$ & $0.06 \pm 0.00$ & ND \\
\hline PFDoA & ND & ND & ND \\
\hline PFTrA & ND & ND & ND \\
\hline PFTeDA & ND & ND & ND \\
\hline PFHxDA & $0.07 \pm 0.04$ & $0.06 \pm 0.04$ & $0.05 \pm 0.01$ \\
\hline PFPrS & ND & ND & ND \\
\hline PFBS & $0.07 \pm 0.01$ & $0.05 \pm 0.00$ & $0.13 \pm 0.04$ \\
\hline PFPeS & ND. & ND. & ND. \\
\hline PFHxS & $0.01 \pm 0.02$ & ND & $0.05 \pm 0.00$ \\
\hline PFHpS & ND & ND & ND \\
\hline PFOS & $0.50 \pm 0.12$ & $0.79 \pm 0.03$ & $1.01 \pm 0.04$ \\
\hline PFNS & ND & ND & ND \\
\hline PFDS & $0.05 \pm 0.01$ & $0.99 \pm 0.08$ & $0.02 \pm 0.00$ \\
\hline PFDos & ND & ND & ND \\
\hline 3:3 FTCA & ND & ND & ND \\
\hline 4:3 FTCA & ND & ND & ND \\
\hline 5:3 FTCA & ND & ND & ND \\
\hline 7:3 FTCA & ND & ND & ND \\
\hline 6:2 FTUA & ND & ND & ND \\
\hline 8:2 FTUA & ND & ND & ND \\
\hline 10:2 FTUA & ND & ND & ND \\
\hline 42 FTSA & ND & ND & ND \\
\hline $6: 2 F T S A$ & $0.13 \pm 0.03$ & ND & ND \\
\hline 8:2 FTSA & $0.02 \pm 0.03$ & ND & ND \\
\hline 10:2 FTSA & ND & ND & ND \\
\hline $6: 2$ FTAB & ND & ND & ND \\
\hline 5:3 FTB & ND & ND & ND \\
\hline $5: 1: 2$ FTB & ND & ND & ND \\
\hline FBSA & ND & ND & ND \\
\hline FHXSA & ND & ND & ND \\
\hline FOSA & ND & ND & ND \\
\hline FOSAA & ND & ND & ND \\
\hline MeFOSAA & $2.05 \pm 0.23$ & $1.86 \pm 0.10$ & $2.14 \pm 0.03$ \\
\hline EtFOSA & ND & ND & ND \\
\hline MeFOSA & ND & ND & ND \\
\hline
\end{tabular}




\begin{tabular}{|c|c|c|c|}
\hline EtFOSAA & ND & ND & ND \\
\hline $\begin{array}{l}\text { PFHxSAm(AmPr- } \\
\text { FHxSA) } \\
\text { PFH } x \text { SAmS(N-TAmP- }\end{array}$ & ND & ND & ND \\
\hline $\begin{array}{l}\text { FHXSA) } \\
\text { PFOAB(N-CMAmP- }\end{array}$ & ND & ND & ND \\
\hline $\begin{array}{l}\text { FOAd) } \\
\text { PFOANO(N-OxAmP- }\end{array}$ & ND & ND & ND \\
\hline FOAd) & ND & ND & ND \\
\hline $\begin{array}{l}\text { PFOSAm(AmPr-FOSA) } \\
\text { PFOSB(N-CMAmP- }\end{array}$ & ND & ND & ND \\
\hline $\begin{array}{l}\text { FOSA) } \\
\text { PFOSNO(N-OxAmP- }\end{array}$ & ND & ND & ND \\
\hline $\begin{array}{l}\text { FOSA) } \\
\text { PFOSAmS(N-TAmP- }\end{array}$ & ND & ND & ND \\
\hline FOSA) & ND & ND & $0.06 \pm 0.00$ \\
\hline PFECHS & ND & ND & $\mathrm{ND}$ \\
\hline
\end{tabular}


Table S5. The spike recovery (mean \pm SE, \%) and matrix effects (mean \pm SE, $\%$ ) of 53 quantitative PFAS analytes in three types of soil matrixes.

(a) Spike recovery of 53 quantitative PFAS analytes in three types of soil matrixes. These soils were extracted by $\mathrm{MeOH}$ with the $100 \mathrm{mM}$ AA method.

\begin{tabular}{|c|c|c|c|}
\hline PFAS analyte & 1R soil (\%) & $2 \mathrm{~N}$ soil(\%) & 3F soil (\%) \\
\hline PFPrA & $87 \pm 14$ & $96 \pm 7$ & $85 \pm 15$ \\
\hline PFBA & $81 \pm 5$ & $95 \pm 17$ & $92 \pm 9$ \\
\hline PFPeA & $81 \pm 9$ & $90 \pm 10$ & $90 \pm 7$ \\
\hline PFHxA & $81 \pm 9$ & $88 \pm 9$ & $90 \pm 7$ \\
\hline PFHpA & $79 \pm 8$ & $87 \pm 10$ & $89 \pm 6$ \\
\hline PFOA & $80 \pm 7$ & $89 \pm 10$ & $87 \pm 6$ \\
\hline PFNA & $79 \pm 8$ & $91 \pm 9$ & $87 \pm 6$ \\
\hline PFDA & $80 \pm 8$ & $87 \pm 10$ & $86 \pm 7$ \\
\hline PFUnA & $80 \pm 8$ & $90 \pm 10$ & $88 \pm 6$ \\
\hline PFDoA & $78 \pm 6$ & $89 \pm 10$ & $88 \pm 8$ \\
\hline PFTrDA & $79 \pm 6$ & $90 \pm 7$ & $85 \pm 6$ \\
\hline PFTeDA & $80 \pm 8$ & $88 \pm 10$ & $86 \pm 7$ \\
\hline PFHxDA & $68 \pm 5$ & $68 \pm 10$ & $67 \pm 12$ \\
\hline PFPrS & $78 \pm 7$ & $89 \pm 10$ & $87 \pm 7$ \\
\hline PFBS & $81 \pm 9$ & $93 \pm 10$ & $88 \pm 6$ \\
\hline PFPeS & $78 \pm 7$ & $89 \pm 10$ & $87 \pm 7$ \\
\hline PFHXS & $79 \pm 8$ & $90 \pm 10$ & $87 \pm 7$ \\
\hline PFHpS & $78 \pm 8$ & $87 \pm 9$ & $89 \pm 7$ \\
\hline PFOS & $80 \pm 7$ & $91 \pm 10$ & $88 \pm 7$ \\
\hline PFNS & $80 \pm 8$ & $87 \pm 8$ & $88 \pm 8$ \\
\hline PFDS & $79 \pm 8$ & $89 \pm 8$ & $87 \pm 8$ \\
\hline PFDoS & $78 \pm 7$ & $85 \pm 9$ & $88 \pm 10$ \\
\hline $4: 2$ FTSA & $83 \pm 6$ & $94 \pm 11$ & $87 \pm 7$ \\
\hline $6: 2$ FTSA & $81 \pm 7$ & $92 \pm 11$ & $83 \pm 6$ \\
\hline 8:2FTSA & $83 \pm 4$ & $90 \pm 11$ & $87 \pm 6$ \\
\hline 10:2 FTSA & $81 \pm 8$ & $88 \pm 8$ & $87 \pm 7$ \\
\hline 3:3 FTCA & $73 \pm 7$ & $85 \pm 12$ & $80 \pm 7$ \\
\hline 4:3 FTCA & $76 \pm 9$ & $85 \pm 7$ & $83 \pm 4$ \\
\hline 5:3 FTCA & $79 \pm 10$ & $87 \pm 6$ & $79 \pm 8$ \\
\hline 7:3 FTCA & $77 \pm 10$ & $89 \pm 4$ & $79 \pm 8$ \\
\hline 6:2 FTUA & $80 \pm 7$ & $89 \pm 10$ & $86 \pm 8$ \\
\hline 8:2 FTUA & $81 \pm 8$ & $91 \pm 9$ & $85 \pm 7$ \\
\hline 10:2 FTUA & $80 \pm 8$ & $86 \pm 11$ & $87 \pm 8$ \\
\hline $6: 2$ FTAB & $73 \pm 5$ & $70 \pm 13$ & $56 \pm 5$ \\
\hline 5:3 FTB & $93 \pm 10$ & $86 \pm 15$ & $102 \pm 5$ \\
\hline $5: 1: 2$ FTB & $100 \pm 8$ & $88 \pm 11$ & $104 \pm 2$ \\
\hline FBSA & $78 \pm 9$ & $86 \pm 9$ & $82 \pm 7$ \\
\hline $\mathrm{FH} \times \mathrm{SA}$ & $77 \pm 8$ & $86 \pm 11$ & $86 \pm 6$ \\
\hline FOSA & $77 \pm 8$ & $86 \pm 10$ & $86 \pm 8$ \\
\hline FOSAA & $70 \pm 7$ & $78 \pm 8$ & $62 \pm 5$ \\
\hline MeFOSAA & $75 \pm 11$ & $93 \pm 12$ & $81 \pm 8$ \\
\hline EtFOSAA & $75 \pm 10$ & $94 \pm 15$ & $80 \pm 5$ \\
\hline MeFOSA & $63 \pm 6$ & $67 \pm 7$ & $79 \pm 9$ \\
\hline EtFOSA & $62 \pm 5$ & $68 \pm 8$ & $79 \pm 9$ \\
\hline PFOAB & $78 \pm 5$ & $75 \pm 5$ & $68 \pm 4$ \\
\hline PFOSB & $72 \pm 5$ & $75 \pm 11$ & $68 \pm 6$ \\
\hline PFOANO & $81 \pm 6$ & $99 \pm 14$ & $82 \pm 7$ \\
\hline PFOSNO & $75 \pm 6$ & $91 \pm 13$ & $90 \pm 9$ \\
\hline PFOSAm & $73 \pm 6$ & $91 \pm 15$ & $86 \pm 5$ \\
\hline PFHxSAm & $80 \pm 4$ & $97 \pm 13$ & $84 \pm 5$ \\
\hline PFOSAmS & $73 \pm 5$ & $87 \pm 15$ & $74 \pm 4$ \\
\hline PFHxSAmS & $82 \pm 5$ & $88 \pm 10$ & $71 \pm 5$ \\
\hline PFECHS & $78 \pm 8$ & $89 \pm 11$ & $88 \pm 7$ \\
\hline
\end{tabular}


(b) Matrix effects (mean \pm SE, \%) of 53 quantitative PFAS in soils when a solvent-based calibration curve was used.

\begin{tabular}{|c|c|c|c|}
\hline \multirow{2}{*}{ PFAS analyte } & \multicolumn{3}{|c|}{ Matrix effects in different soil matrixes (Mean $\pm \mathrm{SE}, \%$ ) } \\
\hline & 1R soil & $2 \mathrm{~N}$ soil & 3F soil \\
\hline PFBA & $16 \pm 2$ & $1 \pm 13$ & $9 \pm 6$ \\
\hline PFPeA & $8 \pm 3$ & $-5 \pm 11$ & $6 \pm 3$ \\
\hline PFHXA & $9 \pm 3$ & $-3 \pm 13$ & $9 \pm 5$ \\
\hline PFHpA & $16 \pm 2$ & $1 \pm 16$ & $14 \pm 5$ \\
\hline PFOA & $11 \pm 2$ & $-1 \pm 14$ & $14 \pm 4$ \\
\hline PFNA & $9 \pm 3$ & $-4 \pm 12$ & $11 \pm 4$ \\
\hline PFDA & $7 \pm 2$ & $-2 \pm 13$ & $10 \pm 5$ \\
\hline PFUnA & $6 \pm 3$ & $-6 \pm 13$ & $8 \pm 3$ \\
\hline PFDoA & $8 \pm 3$ & $-5 \pm 11$ & $8 \pm 3$ \\
\hline PFTrDA & $6 \pm 2$ & $-9 \pm 10$ & $7 \pm 4$ \\
\hline PFTeDA & $8 \pm 3$ & $-7 \pm 10$ & $7 \pm 2$ \\
\hline PFHxDA & $7 \pm 2$ & $-5 \pm 12$ & $8 \pm 4$ \\
\hline PFPrS & $12 \pm 2$ & $3 \pm 12$ & $12 \pm 1$ \\
\hline PFBS & $9 \pm 7$ & $-5 \pm 14$ & $11 \pm 7$ \\
\hline PFPeS & $11 \pm 1$ & $-3 \pm 14$ & $12 \pm 6$ \\
\hline PFHxS & $9 \pm 1$ & $-5 \pm 12$ & $10 \pm 4$ \\
\hline PFHpS & $8 \pm 1$ & $-4 \pm 13$ & $8 \pm 5$ \\
\hline PFOS & $14 \pm 4$ & $6 \pm 17$ & $21 \pm 4$ \\
\hline PFNS & $10 \pm 2$ & $-1 \pm 12$ & $11 \pm 3$ \\
\hline PFDS & $10 \pm 3$ & $-1 \pm 12$ & $12 \pm 4$ \\
\hline PFDoS & $10 \pm 3$ & $1 \pm 10$ & $12 \pm 3$ \\
\hline $4: 2$ FTSA & $10 \pm 7$ & $-5 \pm 13$ & $11 \pm 7$ \\
\hline 6:2 FTSA & $26 \pm 5$ & $10 \pm 13$ & $22 \pm 20$ \\
\hline 8:2 FTSA & $9 \pm 3$ & $-4 \pm 12$ & $8 \pm 5$ \\
\hline 10:2 FTSA & $9 \pm 4$ & $-2 \pm 12$ & $9 \pm 4$ \\
\hline 3:3 FTCA & $-1 \pm 2$ & $-7 \pm 12$ & $6 \pm 3$ \\
\hline 4:3 FTCA & $7 \pm 6$ & $-6 \pm 14$ & $7 \pm 9$ \\
\hline 5:3 FTCA & $4 \pm 7$ & $-7 \pm 12$ & $8 \pm 8$ \\
\hline 7:3 FTCA & $4 \pm 7$ & $-8 \pm 12$ & $6 \pm 5$ \\
\hline 6:2 FTUA & $10 \pm 3$ & $-2 \pm 12$ & $10 \pm 2$ \\
\hline 8:2 FTUA & $10 \pm 2$ & $-4 \pm 12$ & $11 \pm 4$ \\
\hline 10:2 FTUA & $12 \pm 3$ & $1 \pm 15$ & $12 \pm 2$ \\
\hline 6:2 FTAB & $16 \pm 4$ & $1 \pm 9$ & $21 \pm 14$ \\
\hline $5: 3$ FTB & $-22 \pm 5$ & $-21 \pm 3$ & $-24 \pm 7$ \\
\hline $5: 1: 2$ FTB & $-34 \pm 4$ & $-19 \pm 3$ & $-19 \pm 5$ \\
\hline PFOAB & $10 \pm 1$ & $-2 \pm 13$ & $11 \pm 3$ \\
\hline PFOANO & $10 \pm 1$ & $-6 \pm 11$ & $13 \pm 1$ \\
\hline PFHxSAm & $-8 \pm 12$ & $9 \pm 2$ & $-13 \pm 5$ \\
\hline PFHxSAmS & $-14 \pm 10$ & $11 \pm 4$ & $-9 \pm 6$ \\
\hline PFECHS & $7 \pm 0$ & $-5 \pm 13$ & $8 \pm 4$ \\
\hline PFOSAM & $22 \pm 7$ & $1 \pm 14$ & $11 \pm 2$ \\
\hline PFOSB & $19 \pm 5$ & $1 \pm 19$ & $12 \pm 2$ \\
\hline PFOSNO & $17 \pm 5$ & $0 \pm 12$ & $10 \pm 2$ \\
\hline PFOSAmS & $23 \pm 8$ & $0 \pm 15$ & $14 \pm 1$ \\
\hline FBSA & $11 \pm 9$ & $-8 \pm 11$ & $11 \pm 7$ \\
\hline $\mathrm{FH} x \mathrm{SA}$ & $11 \pm 6$ & $-5 \pm 12$ & $9 \pm 5$ \\
\hline FOSA & $10 \pm 3$ & $-3 \pm 12$ & $11 \pm 4$ \\
\hline FOSAA & $14 \pm 1$ & $-2 \pm 11$ & $10 \pm 2$ \\
\hline MeFOSAA & $8 \pm 2$ & $-12 \pm 18$ & $7 \pm 2$ \\
\hline MeFOSA & $7 \pm 5$ & $-5 \pm 9$ & $10 \pm 3$ \\
\hline EtFOSA & $9 \pm 3$ & $-3 \pm 13$ & $9 \pm 5$ \\
\hline EtFOSAA & $3 \pm 4$ & $-16 \pm 8$ & $4 \pm 5$ \\
\hline
\end{tabular}


Table S6. The spike recoveries (mean \pm SE, \%) of 53 quantitative PFAS in three types of soils with the new exhaustive extraction method.

These soils were extracted using the comprehensive method (extraction by methanol with $400 \mathrm{mM} \mathrm{NaOH}$ for two cycles followed by methanol with $400 \mathrm{mM} \mathrm{HCl}$ for one cycle). Absolute recoveries lower than $60 \%$ are highlighted in red font. The low recoveries of FTUCAs are probably due to a reaction with methanol in the presence of a base to form methoxy-substituted unsaturated telomer acids. ${ }^{12}$

\begin{tabular}{llll}
\hline PFAS analyte & $\mathbf{1 R}$ soil & $\mathbf{2 N}$ soil & 3F soil \\
\hline PFPrA & $104 \pm 32$ & $95 \pm 23$ & $112 \pm 5$ \\
PFBA & $98 \pm 5$ & $101 \pm 4$ & $122 \pm 21$ \\
PFPeA & $99 \pm 1$ & $95 \pm 4$ & $111 \pm 5$ \\
PFHXA & $91 \pm 8$ & $93 \pm 5$ & $117 \pm 2$ \\
PFHpA & $106 \pm 6$ & $92 \pm 5$ & $112 \pm 4$ \\
PFOA & $116 \pm 8$ & $92 \pm 5$ & $116 \pm 2$ \\
PFNA & $103 \pm 7$ & $93 \pm 2$ & $112 \pm 1$ \\
PFDA & $106 \pm 4$ & $92 \pm 2$ & $122 \pm 1$ \\
PFUnA & $100 \pm 5$ & $98 \pm 4$ & $115 \pm 2$ \\
PFDoA & $110 \pm 5$ & $93 \pm 3$ & $113 \pm 1$ \\
PFTrDA & $100 \pm 9$ & $95 \pm 2$ & $111 \pm 2$ \\
PFTeDA & $107 \pm 6$ & $94 \pm 2$ & $113 \pm 1$ \\
PFHxDA & $100 \pm 4$ & $86 \pm 2$ & $103 \pm 1$ \\
PFPrS & $106 \pm 9$ & $84 \pm 2$ & $111 \pm 3$ \\
PFBS & $103 \pm 7$ & $91 \pm 1$ & $116 \pm 1$ \\
PFPeS & $108 \pm 7$ & $93 \pm 0$ & $115 \pm 2$ \\
PFHxS & $103 \pm 9$ & $87 \pm 2$ & $111 \pm 1$ \\
PFHpS & $102 \pm 7$ & $93 \pm 2$ & $112 \pm 3$ \\
PFOS & $100 \pm 4$ & $88 \pm 7$ & $121 \pm 3$ \\
PFNS & $105 \pm 12$ & $93 \pm 1$ & $114 \pm 3$ \\
PFDS & $104 \pm 5$ & $92 \pm 2$ & $109 \pm 0$ \\
PFDoS & $101 \pm 6$ & $90 \pm 5$ & $112 \pm 4$ \\
PFECHS & $103 \pm 9$ & $85 \pm 2$ & $112 \pm 0$ \\
42 FTSA & $107 \pm 11$ & $120 \pm 0$ & $113 \pm 16$ \\
62 FTSA & $112 \pm 15$ & $125 \pm 5$ & $122 \pm 9$ \\
82 FTSA & $106 \pm 8$ & $120 \pm 9$ & $129 \pm 12$ \\
10:2 FTSA & $104 \pm 9$ & $127 \pm 9$ & $115 \pm 8$ \\
3:3 FTCA & $110 \pm 18$ & $86 \pm 4$ & $127 \pm 9$ \\
4:3 FTCA & $107 \pm 1$ & $41 \pm 2$ & $116 \pm 6$ \\
5:3 FTCA & $96 \pm 6$ & $108 \pm 7$ & $117 \pm 0$ \\
7:3 FTCA & $109 \pm 9$ & $100 \pm 8$ & $110 \pm 8$ \\
6:2 FTUA & $24 \pm 3$ & $76 \pm 1$ & $39 \pm 1$ \\
8:2 FTUA & $27 \pm 1$ & $40 \pm 3$ & $41 \pm 2$ \\
10:2 FTUA & $30 \pm 5$ & $63 \pm 4$ & $44 \pm 2$ \\
6:2 FTAB & $99 \pm 6$ & $84 \pm 5$ & $103 \pm 3$ \\
5:3 FTB & $98 \pm 8$ & $90 \pm 5$ & $110 \pm 7$ \\
5:1:2 FTB & $77 \pm 1$ & $98 \pm 0$ & $91 \pm 12$ \\
FBSA & $110 \pm 7$ & $102 \pm 2$ & $117 \pm 1$ \\
FHxSA & $107 \pm 4$ & $107 \pm 1$ & $120 \pm 5$ \\
FOSA & $107 \pm 8$ & $105 \pm 1$ & $114 \pm 1$ \\
FOSAA & $53 \pm 6$ & $40 \pm 6$ & $55 \pm 5$ \\
MeFOSA & $102 \pm 18$ & $76 \pm 12$ & $125 \pm 0$ \\
EtFOSA & $98 \pm 36$ & $104 \pm 3$ & $124 \pm 11$ \\
MeFOSAA & $132 \pm 8$ & $82 \pm 22$ & $144 \pm 11$ \\
EtFOSAA & $20 \pm 28$ & $76 \pm 18$ & $89 \pm 20$ \\
PFHxSAm & $108 \pm 7$ & $95 \pm 19$ & $102 \pm 7$ \\
PFOSAm & $109 \pm 6$ & $66 \pm 11$ & $107 \pm 3$ \\
PFHxSAmS & $108 \pm 10$ & $95 \pm 11$ & $108 \pm 4$ \\
& & &
\end{tabular}




\begin{tabular}{llll} 
PFOSAmS & $119 \pm 8$ & $75 \pm 10$ & $102 \pm 0$ \\
PFOAB & $94 \pm 14$ & $89 \pm 32$ & $92 \pm 10$ \\
PFOSB & $115 \pm 11$ & $79 \pm 34$ & $104 \pm 0$ \\
PFOANO & $101 \pm 10$ & $73 \pm 3$ & $96 \pm 3$ \\
PFOSNO & $107 \pm 10$ & $71 \pm 5$ & $96 \pm 2$ \\
\hline
\end{tabular}


Table S7. PFAS analyte list (target and suspect-screening) for field soil and groundwater samples.

\begin{tabular}{|c|c|c|c|c|}
\hline $\begin{array}{l}\text { Class } \\
\text { Number }\end{array}$ & Analyte acronym and structure & $\begin{array}{l}\text { Perfluoroalkyl } \\
\text { chain, } \mathbf{n}\end{array}$ & Reference & Full Name \\
\hline \multicolumn{5}{|l|}{ PFCA } \\
\hline 1 & PFC & $2-13,15$ & 11-13 & Perfluoroalkyl carboxylic acid \\
\hline \multicolumn{5}{|l|}{ PFSA } \\
\hline 2 & $\begin{array}{l}\text { PFSA } \\
\mathrm{F}\left[\begin{array}{l}\mathrm{F} \\
\left.\prod_{\mathrm{F}}\right]_{\mathrm{n}}\end{array}\right.\end{array}$ & $2-10$ & $11-14$ & Perfluoroalkyl sulfonic acid \\
\hline \multicolumn{5}{|c|}{ Fluorotelomer (FT)-derived compounds } \\
\hline 3 & n:2 FTUA & $6,8,10$ & 12 & n:2 fluorotelomer unsaturated acid \\
\hline 4 & n:3 FTCA & $3-11$ & 12 & n:3 fluorotelomer carboxylic acid \\
\hline 5 & $\mathrm{n}: 2 \mathrm{~F}$ & $\begin{array}{l}4,6,8,10 \\
12,14\end{array}$ & $7,11,15$ & n:2 fluorotelomer sulfonic acid \\
\hline 6 & n:2 FTAB & $\begin{array}{l}4,6,8,10 \\
12,14,16\end{array}$ & $7,11,14,16$ & $\begin{array}{l}\mathrm{n}: 2 \text { fluorotelomer } \\
\text { sulfonamide betaine }\end{array}$ \\
\hline 7 & $n: 2$ FTNO & $6,8,10$ & 8,17 & $\begin{array}{l}\text { n:2 fluorotelomer } \\
\text { sulfonamide amine oxide }\end{array}$ \\
\hline
\end{tabular}




\begin{tabular}{|c|c|c|c|c|}
\hline $\begin{array}{l}\text { Class } \\
\text { Number }\end{array}$ & Analyte acronym and structure & $\begin{array}{l}\text { Perfluoroalkyl } \\
\text { chain, } \mathbf{n}\end{array}$ & Reference & Full Name \\
\hline 8 & n:2 FTA (or FTAA, or & $4,6,8,10,12$ & $3,7,11,14,16$ & $\mathrm{n}: 2$ fluorotelomer sulfonamide amine \\
\hline 9 & $\mathrm{n}: 2$ demethyl-FTA & $6,8,10,12$ & 3 & $\mathrm{n}: 2$ demethyl-fluorotelomer sulfonamide amine \\
\hline 10 & & $\begin{array}{l}5,7,9,11 \\
13,15\end{array}$ & $7,11,14,16$ & $\mathrm{n}: 3$ fluorotelomer betaine \\
\hline 11 & 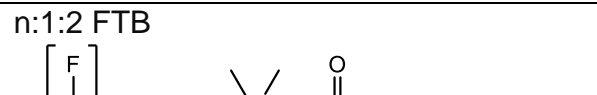 & $\begin{array}{l}5,7,9,11 \\
13,15\end{array}$ & $7,11,14,16$ & $\mathrm{n}: 1: 2$ fluorotelomer betaine \\
\hline 12 & $\begin{array}{l}\mathrm{n}: 2 \text { FTSAS } \\
{\left[\begin{array}{c}\mathrm{F} \\
\end{array}\right.}\end{array}$ & $\begin{array}{l}2,4,6,8,10 \\
12,14\end{array}$ & $7,11,14-16,18$ & n:2 fluorotelomermercaptoalkylamido sulfonate \\
\hline 13 & $\begin{array}{c}\mathrm{n}: 2 \text { FTSAS-SO (or n:2 FTSAS-sulfoxide) } \\
{[\mathrm{F}]}\end{array}$ & $4,6,8,10,12$ & $14-16,19$ & $\begin{array}{l}\mathrm{n}: 2 \text { fluorotelomermercaptoalkylamido sulfonate- } \\
\text { sulfoxide }\end{array}$ \\
\hline 14 & $\mathrm{n}: 2$ FTSAS-SO 2 (or n:2 FASO2PA-MePS) & $4,6,8,10,12$ & 20 & $\begin{array}{l}\mathrm{n}: 2 \text { fluorotelomer mercaptoalkylamido sulfonate } \\
\text { sulfone or } \mathrm{n}: 2 \text { tridecafluoroalkyl sulfonyl (SO2) } \\
\text { propanoamido-methylpropylsulfonate }\end{array}$ \\
\hline
\end{tabular}




\begin{tabular}{|c|c|c|c|c|}
\hline $\begin{array}{l}\text { Class } \\
\text { Number }\end{array}$ & Analyte acronym and structure & $\begin{array}{l}\text { Perfluoroalkyl } \\
\text { chain, } \mathbf{n}\end{array}$ & Reference & Full Name \\
\hline 15 & $\mathrm{n}: 2: 2$ FTSC (or n:2 FTS- $\left.\mathrm{C}_{2} \mathrm{H}_{4}-\mathrm{COOH}\right)$ & $\begin{array}{l}4,6,8,10 \\
12,14\end{array}$ & $3,12,16$ & $\begin{array}{l}\mathrm{n}: 2: 2 \text { fluorotelomer thioether propanoate } \\
(\mathrm{n}: 2: 2 \text { fluorotelomer mercaptoalkyl carboxylate) }\end{array}$ \\
\hline 16 & $\mathrm{n}: 2: 1$ FTSC (or n:2 FTS- $\mathrm{CH}_{2}-\mathrm{COOH}$ ) & $\begin{array}{l}4,6,8,10,12 \\
14\end{array}$ & $3,12,16$ & $\begin{array}{l}\mathrm{n}: 2: 1 \text { fluorotelomer thioether ethanoate } \\
\text { ( } \mathrm{n}: 2: 1 \text { fluorotelomer mercaptoalkyl carboxylate) }\end{array}$ \\
\hline 17 & & $4,6,8,10,12$ & $3,7,11,14,16$ & $\mathrm{n}: 2$ fluorotelomer thiohydroxyammonium \\
\hline 18 & n:2 FTSHA-SO (or n:2 FTSHA-sulfoxide) & $4,6,8,10$ & 12,16 & $\mathrm{n}: 2$ fluorotelomer thiohydroxyammonium-sulfoxide \\
\hline 19 & $\begin{array}{l}\mathrm{n}: 2 \text { FTSAAC } \\
{[\mathrm{F}]}\end{array}$ & $6,8,10$ & 3,14 & $\begin{array}{l}\mathrm{n}: 2 \text { fluorotelomer thioether amino carboxylic acids } \\
\text { ( } \mathrm{n}: 2 \text { fluorotelomer thioalkylamido amine carboxylate) }\end{array}$ \\
\hline 20 & $\mathrm{n}: 2$ FTSAB & $\begin{array}{l}4,6,8,10 \\
12,14\end{array}$ & $7,11,14,16$ & $\mathrm{n}: 2$ fluorotelomer sulfonamide betaines \\
\hline 21 & $\begin{array}{l}\mathrm{n}: 2 \text { FTSAA } \\
{[\mathrm{F}]}\end{array}$ & $\begin{array}{l}4,6,8,10 \\
12,14\end{array}$ & 16 & $\mathrm{n}: 2$ fluorotelomer thio alkylamine \\
\hline 22 & $\mathrm{n}: 2$ FTSAAmS or $\mathrm{n}: 2$ FTStrA & $\begin{array}{l}2,4,6,8,10 \\
12,14\end{array}$ & 16 & $\begin{array}{l}\mathrm{n}: 2 \text { fluorotelomer thio alkylamido ammonium or } \mathrm{n}: 2 \\
\text { fluorotelomer thio trialkylamine }\end{array}$ \\
\hline
\end{tabular}




\begin{tabular}{|c|c|c|c|c|}
\hline $\begin{array}{l}\text { Class } \\
\text { Number }\end{array}$ & Analyte acronym and structure & $\begin{array}{l}\text { Perfluoroalkyl } \\
\text { chain, } \mathbf{n}\end{array}$ & Reference & Full Name \\
\hline 23 & $\mathrm{n}: 2$ FTStrA-SO (or n:2 FTSoAAmS) & $\begin{array}{l}2,4,6,8,10 \\
12,14\end{array}$ & 16 & $\begin{array}{l}\mathrm{n}: 2 \text { fluorotelomer sulfinyl alkylamido ammonium or } \\
\mathrm{n}: 2 \text { fluorotelomer thio trialkylamine sulfoxide }\end{array}$ \\
\hline 24 & $\mathrm{n}: 2$ FTSAm (or $\mathrm{n}: 2$ FASA) & $\begin{array}{l}6,8,10,12 \\
14\end{array}$ & 3,21 & $\mathrm{n}: 2$ fluorotelomer sulfonamide \\
\hline 25 & $\mathrm{n}: 2 \mathrm{FTSO}_{2} \mathrm{PA}$ & $4,6,8$ & 22 & $\begin{array}{l}\mathrm{n}: 2 \text { fluorotelomersulfonyl }\left(\mathrm{O}_{2}\right) \\
\text { propanoic acid }\end{array}$ \\
\hline 26 & & $4-8$ & 22 & $\begin{array}{l}\text { 1-hydroxy }(\mathrm{HO})-\mathrm{n}: 2 \text { fluorotelomer } \\
\text { sulfonate }\end{array}$ \\
\hline 27 & CMAmEt-FA & $6,8,10$ & 22 & $\begin{array}{l}\text { Carboxymethyldimethylammonioethyl- } \\
\text { perfluoroalkane }\end{array}$ \\
\hline 28 & CmAmB-FA (or n:4 FTB) & $4,6,8$ & 22 & $\begin{array}{l}\text { Carboxymethyldimethylammoniobutyl- } \\
\text { perfluoroalkane }\end{array}$ \\
\hline \multicolumn{5}{|c|}{ ECF-derived sulfonamides and amides } \\
\hline 29 & FASA & $3-10$ & $12,20,22$ & $\begin{array}{l}\text { perfluoroalkane } \\
\text { sulfonamide }\end{array}$ \\
\hline
\end{tabular}




\begin{tabular}{|c|c|c|c|c|}
\hline $\begin{array}{l}\text { Class } \\
\text { Number }\end{array}$ & Analyte acronym and structure & $\begin{array}{l}\text { Perfluoroalkyl } \\
\text { chain, } \mathbf{n}\end{array}$ & Reference & Full Name \\
\hline & 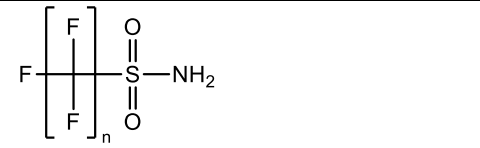 & & & \\
\hline 30 & 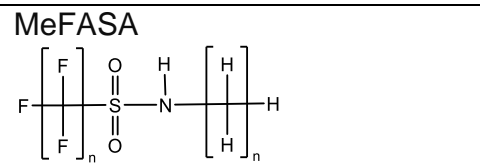 & 6,8 & 1 & N-methyl-perfluoroalkyl sulfonamide \\
\hline 31 & 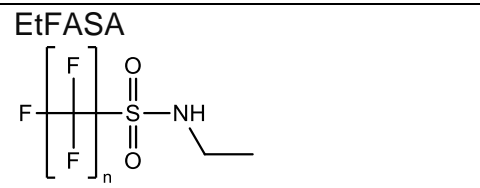 & 6,8 & 12 & N-ethyl-perfluoroalkyl sulfonamide \\
\hline 32 & 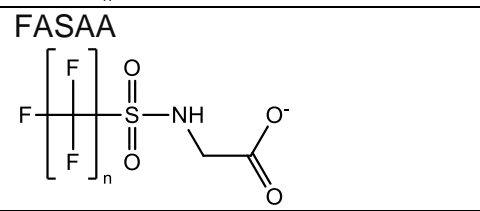 & $4-8$ & 12,22 & Perfluoroalkyl sulfonamidoacetic acid \\
\hline 33 & N-EtFASAA & $2-10$ & 22 & N-ethyl perfluoroalkyl sulfonamide acetic acid \\
\hline 34 & 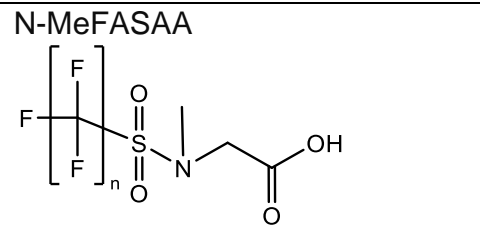 & $3-8$ & 22 & N-methyl perfluoroalkyl sulfonamide acetic acid \\
\hline 35 & 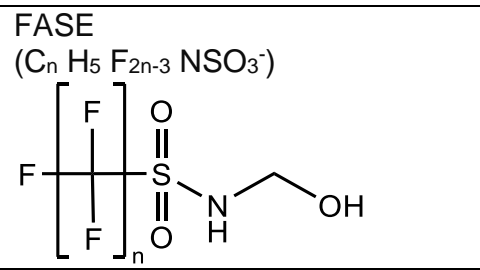 & $4-10$ & 13 & Perfluoroalkyl sulfonamidoethanols \\
\hline 36 & PFSiAs & $4-8$ & 1,13 & Perfuoroalkane sulfinate \\
\hline
\end{tabular}




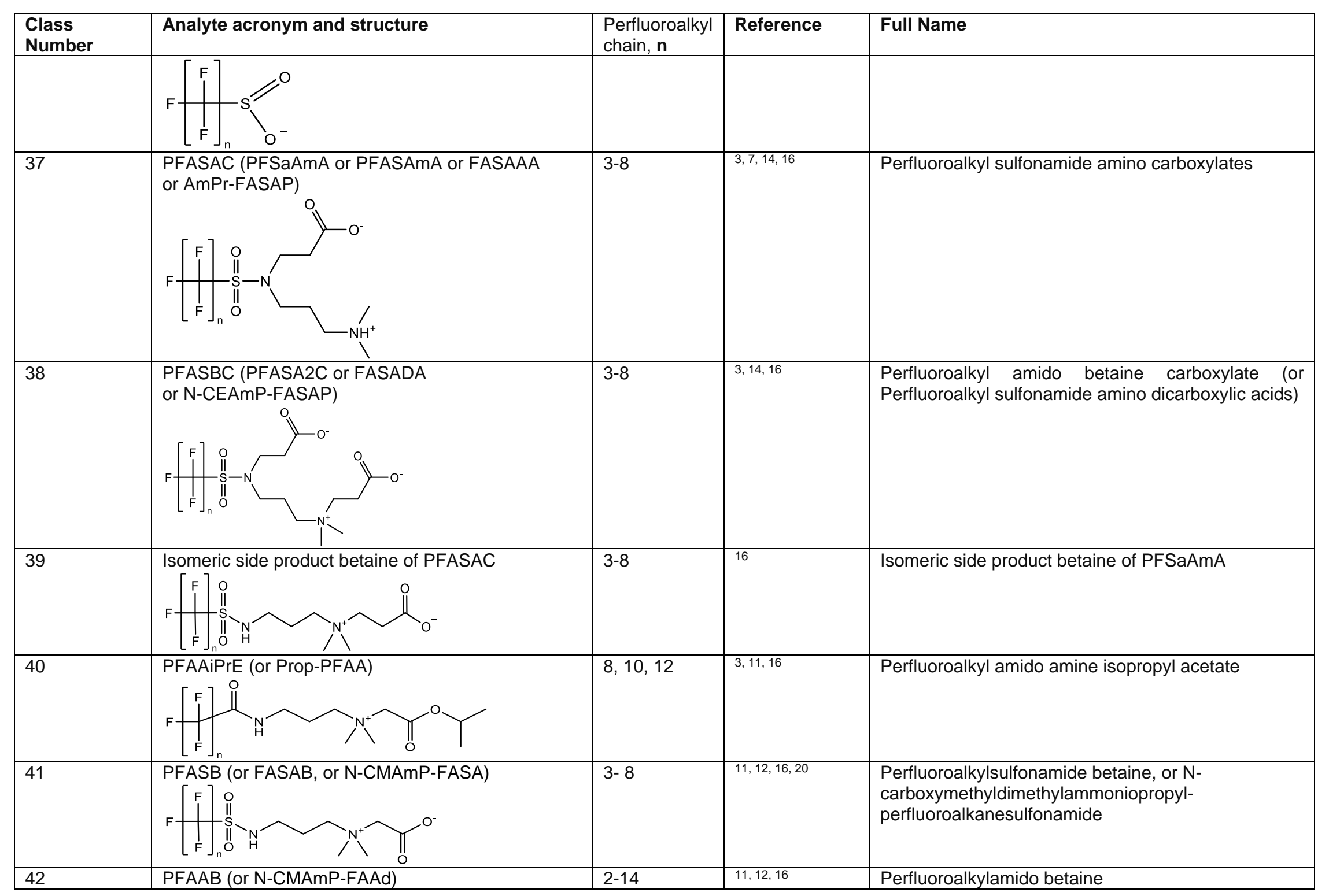




\begin{tabular}{|c|c|c|c|c|}
\hline $\begin{array}{l}\text { Class } \\
\text { Number }\end{array}$ & Analyte acronym and structure & $\begin{array}{l}\text { Perfluoroalkyl } \\
\text { chain, } \mathbf{n}\end{array}$ & Reference & Full Name \\
\hline 43 & AmPr-FASA) & $3-9$ & $7,14,16,20$ & Perfluoroalkyl sulfonamide amines \\
\hline 44 & PFAAAm (or AmPr & 3-14 & 3,16 & $\begin{array}{l}\text { Perfluoroalkyl amido amine } \quad \text { (or } \\
\text { (dimethylamino)propyl)-Perfluoroalkylamide) }\end{array}$ \\
\hline 45 & PFASNO (or N-OxAmP-FASA, OAmPr-FASA) & $4-9$ & 16 & Perfluoroalkyl sulfonamide amine oxide \\
\hline 46 & PFAANO (or N-OxAmP-FAAd, OAmPr-FAAd) & $3-9$ & 3 & Perfluoroalkyl amido amine oxide \\
\hline 47 & (or N-TAmP-FASA) & $3-8$ & 20,23 & $\begin{array}{l}\text { N-trimethylammoniopropyl } \\
\text { Perfluoroalkanesulfonamide }\end{array}$ \\
\hline 48 & PFAAAmS (or N-TAmP-FAAd) & $3-7$ & 12,16 & Perfluoroalkyl-amido ammonium salt \\
\hline 49 & N-SP-FASA & $3-6$ & 22 & $\begin{array}{l}\text { N-sulfopropyl } \\
\text { perfluoroalkanesulfonamide }\end{array}$ \\
\hline
\end{tabular}




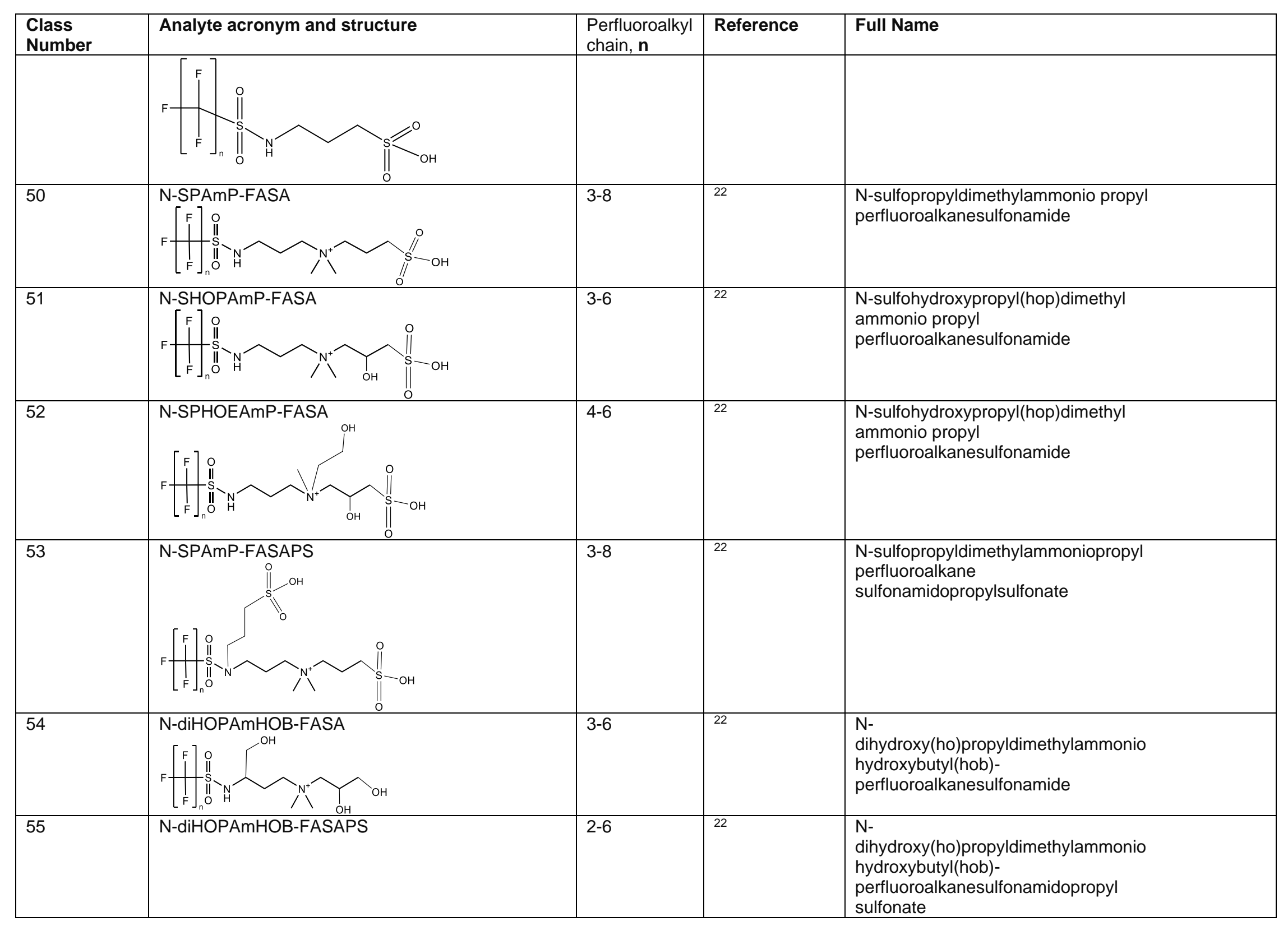




\begin{tabular}{|c|c|c|c|c|}
\hline $\begin{array}{l}\text { Class } \\
\text { Number }\end{array}$ & Analyte acronym and structure & $\begin{array}{l}\text { Perfluoroalkyl } \\
\text { chain, } \mathbf{n}\end{array}$ & Reference & Full Name \\
\hline 56 & $\mathrm{~N}-\mathrm{HOEAmP}$ & $2-8$ & 22 & $\begin{array}{l}\text { N-hydroxyethyl(hoe)dimethylammonio } \\
\text { propyl perfluoroalkanesulfonamido } \\
\text { propylsulfonate }\end{array}$ \\
\hline 57 & 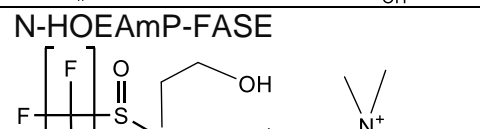 & $2-8$ & 22 & $\begin{array}{l}\text { N-hydroxyethyl(hoe)dimethylammonio } \\
\text { propyl perfluoroalkane } \\
\text { sulfonamidoethanol }\end{array}$ \\
\hline 58 & $\begin{array}{c}\mathrm{N}-\mathrm{HOEAm} \\
{[\mathrm{F}] \mathrm{O}}\end{array}$ & $2-8$ & 22 & $\begin{array}{l}\text { N-hydroxyethyl(hoe)dimethylammonio } \\
\text { propyl perfluoroalkanesulfonamide }\end{array}$ \\
\hline 59 & 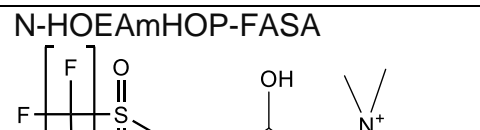 & $4-6$ & 22 & $\begin{array}{l}\text { N-hydroxyethyl(hoe)dimethylammonio } \\
\text { hydroxypropyl(hop) } \\
\text { perfluoroalkanesulfonamide }\end{array}$ \\
\hline 60 & $\begin{array}{l}\text { N-TAmP-N-MeFASA } \\
\text { Pa }\end{array}$ & $4-8$ & 22 & $\begin{array}{l}\text { N-trimethylammoniopropyl n-methyl } \\
\text { perfluoroalkanesulfonamide }\end{array}$ \\
\hline 61 & N-TAmP-F & $3-6$ & 22 & $\begin{array}{l}\text { N-trimethylammoniopropyl } \\
\text { perfluoroalkylsulfonamido propanoic } \\
\text { acid }\end{array}$ \\
\hline 62 & N-CMAmP-FASAP & $4-6$ & 22 & $\begin{array}{l}\mathrm{N} \text { - } \\
\text { carboxymethyldimethylammoniopropyl- } \\
\text { perfluoroalkylsulfonamido propanoic }\end{array}$ \\
\hline
\end{tabular}




\begin{tabular}{|c|c|c|c|c|}
\hline $\begin{array}{l}\text { Class } \\
\text { Number }\end{array}$ & Analyte acronym and structure & $\begin{array}{l}\text { Perfluoroalkyl } \\
\text { chain, } \mathbf{n}\end{array}$ & Reference & Full Name \\
\hline & & & & acid \\
\hline 63 & $\mathrm{~N}-\mathrm{HOEAmP}-\mathrm{FA}$ & $4-6$ & 22 & $\begin{array}{l}\text { N-hydroxyethyl(hoe)dimethylammonio } \\
\text { propyl perfluoroalkanesulfonamido } \\
\text { hydroxy(ho)propyl sulfonate }\end{array}$ \\
\hline 64 & N-SHOPAmP & $2-6$ & 22 & $\begin{array}{l}\text { N-sulfohydroxypropyl(hop)dimethyl } \\
\text { ammonio propyl } \\
\text { perfluoroalkanesulfonamido } \\
\text { hydroxy(HO)Propyl Sulfonate }\end{array}$ \\
\hline 65 & N-AHOB-FA & $5-6$ & 22 & $\begin{array}{l}\text { N-dimethylaminohydroxybutyl(hob)- } \\
\text { perfluoroalkanesulfoamido } \\
\text { propylsulfonate }\end{array}$ \\
\hline 66 & $\begin{array}{l}\text { N-SPAmP-MeFASA } \\
{[F] O}\end{array}$ & $3-6$ & 22 & $\begin{array}{l}\mathrm{N} \text {-sulfopropyldimethylammoniopropyl } \\
\text { methyl perfluoroalkanesulfonamide }\end{array}$ \\
\hline 67 & N-SPAmP-FASAA & $3-6$ & 22 & $\begin{array}{l}\text { N-sulfopropyldimethylammoniopropyl- } \\
\text { perfluoroalkanesulfonamido acetic } \\
\text { acid }\end{array}$ \\
\hline
\end{tabular}




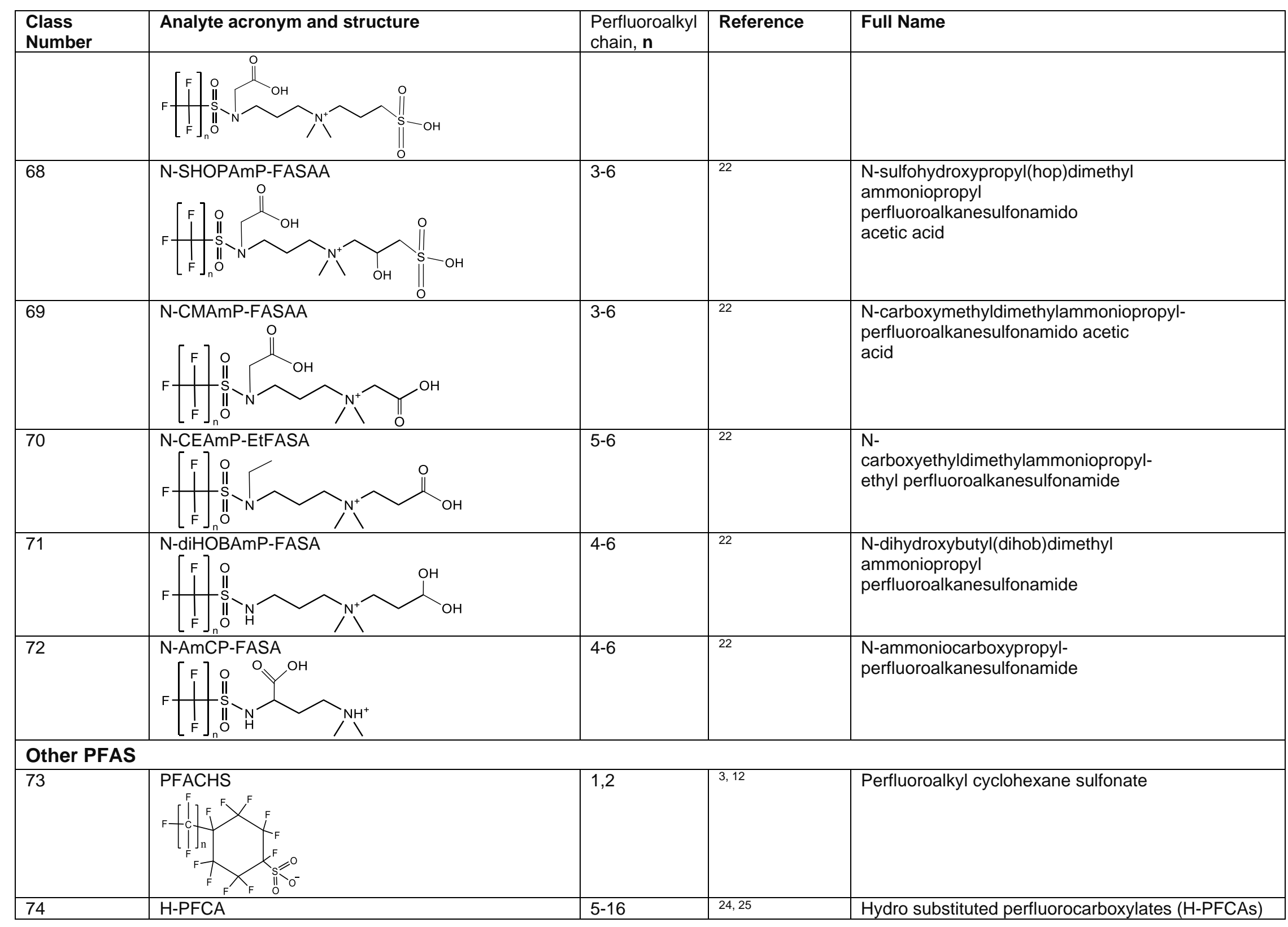




\begin{tabular}{|c|c|c|c|c|}
\hline $\begin{array}{l}\text { Class } \\
\text { Number }\end{array}$ & Analyte acronym and structure & $\begin{array}{l}\text { Perfluoroalkyl } \\
\text { chain, } \mathbf{n}\end{array}$ & Reference & Full Name \\
\hline & $(\mathrm{HCnF} 2 \mathrm{nCOO}-)$ & & & \\
\hline 75 & $\begin{array}{l}\text { Cl-PFCA } \\
\left(\mathrm{ClC}_{n} \mathrm{~F}_{2 n} \mathrm{COO}^{-}\right)\end{array}$ & $4-11$ & 24 & Chlorine substituted perfluorocarboxylates \\
\hline 76 & $\begin{array}{l}\mathrm{PFS} \\
\left(\mathrm{C}_{2} \mathrm{~F}_{5}\left(\mathrm{C}_{2} \mathrm{H}_{4}\right)_{n} \mathrm{CHFOSO}_{3}^{-}\right)\end{array}$ & $1-6$ & 24 & Polyfluorinated sulfonates (PFSs) \\
\hline 77 & $\begin{array}{l}\mathrm{H}-\mathrm{PFE} / \mathrm{As} \\
\left(\mathrm{C}_{\mathrm{n}} \mathrm{F}_{2 \mathrm{n}-2 \mathrm{HO}}\right)\end{array}$ & $7-11$ & 24 & $\begin{array}{l}\text { Unsaturated hydro substituted perfluorinated } \\
\text { ethers/alcohols }\end{array}$ \\
\hline 78 & $\begin{array}{l}\mathrm{Cl}-\mathrm{PFE} / \mathrm{As} \\
\left(\mathrm{ClC}_{n} \mathrm{~F}_{2 \mathrm{n}-2} \mathrm{O}^{-}\right)\end{array}$ & $6-10$ & 24 & $\begin{array}{l}\text { Unsaturated chlorine substituted perfluorinated } \\
\text { ethers/alcohols }\end{array}$ \\
\hline 79 & n+1-F5S-PFAA & $6-8$ & 22 & $\begin{array}{l}\text { ( } \mathrm{n}+1 \text {-Pentafluoro(5)sulfide)- } \\
\text { perfluoroalkanoic acid }\end{array}$ \\
\hline 80 & $\begin{array}{l}\text { Chlorinated PFSAs } \\
\text { (or Cl-PFSA) }\end{array}$ & $3,4,5,6,7,8$ & $1,13,26$ & $\begin{array}{l}\text { Chloro(Cl)- } \\
\text { perfluoroalkanesulfonate }\end{array}$ \\
\hline 81 & $\begin{array}{l}\text { Dichlorinated PFSAs } \\
\left(\mathrm{C}_{n} \mathrm{~F}_{2 \mathrm{n}-1} \mathrm{Cl}_{2} \mathrm{SO}_{3}\right)\end{array}$ & 3,8 & 13 & Chlorinated Perfluoroalkyl Sulphonate \\
\hline 82 & $\begin{array}{l}\text { K-PFSA (or Ketone PFSA) } \\
\left(\mathrm{C}_{n} \mathrm{~F}_{2 \mathrm{n}-1} \mathrm{SO} 4\right)\end{array}$ & $3-13$ & 13,26 & Ketone perfluoroalyl sulfonate \\
\hline 83 & $\begin{array}{c}\text { Ether-PFSA (O-PFSA) } \\
{[}\end{array}$ & $\begin{array}{l}3,4,5,6,7,8 \\
9,10\end{array}$ & 1,26 & Ether perfluoroalkane sulfonate \\
\hline 84 & $\begin{array}{l}\mathrm{PFSA}-\mathrm{Un} \\
\mathrm{C}_{n} \mathrm{~F}_{2 \mathrm{n}-1} \mathrm{SO}_{3}^{-}\end{array}$ & 8 & 13 & Perfluoroalkene sulphonate \\
\hline 85 & n-F5S-PFAS & $3-9$ & 22 & (n-Pentafluoro(5)sulfide)- \\
\hline
\end{tabular}




\begin{tabular}{|c|c|c|c|c|}
\hline $\begin{array}{l}\text { Class } \\
\text { Number }\end{array}$ & Analyte acronym and structure & $\begin{array}{l}\text { Perfluoroalkyl } \\
\text { chain, } \mathbf{n}\end{array}$ & Reference & Full Name \\
\hline & O & & & perfluoroalkane sulfonate \\
\hline 86 & Multiple isomers possible & $1-10$ & 22 & Unsaturated perfluoroalkane sulfonate \\
\hline 87 & Multiple isomers possible & $1-6$ & 22 & $\begin{array}{l}\text { Hydrido-unsaturated perfluoroalkane } \\
\text { sulfonate }\end{array}$ \\
\hline 88 & Multiple isomers possible & $0-8$ & 22 & Hydrido-perfluoroalkane sulfonate \\
\hline 89 & Multiple isomers possible & 5,7 & 22 & $\mathrm{n}: 1$ perfluoroalkanesulfonate \\
\hline 90 & Multiple isomers possible & $0-4^{a}$ & 22 & Oxa-unsaturated-perfluoroalkanoic acid \\
\hline 91 & $\mathrm{C}_{n+8} \mathrm{H}_{16} \mathrm{O}_{2} \mathrm{SN}_{2} \mathrm{~F}_{2 n+1}$ & $6,8,10$ & 22 & Not applicable \\
\hline 92 & $\begin{array}{l}\mathrm{C}_{n+10} \mathrm{H}_{20} \mathrm{O}_{7} \mathrm{SN}_{2} \mathrm{~F}_{2 n+1} \text { or } \\
\mathrm{C}_{n+10} \mathrm{H}_{18} \mathrm{O}_{4} \mathrm{SN}_{2} \mathrm{~F}_{2 \mathrm{n}+1}\end{array}$ & Unknown & 22 & Not applicable \\
\hline 93 & $\mathrm{C}_{n+9} \mathrm{H}_{22} \mathrm{O}_{2} \mathrm{SN}_{2} \mathrm{~F}_{2 n+1}$ & $3,4,6$ & 22 & Not applicable \\
\hline
\end{tabular}


Table S8. Correspondence between native PFAS analytes and isotopically labeled surrogate IS.

\begin{tabular}{|c|c|}
\hline Analyte & Surrogate IS \\
\hline $\begin{array}{l}\text { PFPrA } \\
\text { PFBA }\end{array}$ & $\begin{array}{l}\text { MPFBA } \\
\text { MPFBA }\end{array}$ \\
\hline PFPeA & M5PFhxA \\
\hline PFHxA & M5PFhxA \\
\hline PFHpA & M8PFOA \\
\hline PFOA & M8PFOA \\
\hline PFNA & M9PFNA \\
\hline PFDA & M6PFDA \\
\hline PFUnA & M7PFUnA \\
\hline PFDoA & MPFDoA \\
\hline PFTrDA & M2PFTeDA \\
\hline PFTeDA & M2PFTeDA \\
\hline PFHxDA & M2PFTeDA \\
\hline PFOcDA & M2PFTeDA \\
\hline PFPrS & M3PFBS \\
\hline PFBS & M3PFBS \\
\hline PFPeS & M3PFHxS \\
\hline PFHxS & M3PFHxS \\
\hline PFHpS & M8PFOS \\
\hline PFOS & M8PFOS \\
\hline PFNS & M8PFOS \\
\hline PFDS & M8PFOS \\
\hline PFDos & M8PFOS \\
\hline PFECHS & M3PFHxS \\
\hline FBSA & M8FOSA-I \\
\hline FHxSA & M8FOSA-I \\
\hline FOSA & M8FOSA-I \\
\hline MeFOSA & d-N-MeFOSA-M \\
\hline EtFOSA & d-N-EtFOSA-M \\
\hline FOSAA & d3-N-MeFOSAA \\
\hline MeFOSAA & d3-N-MeFOSAA \\
\hline EtFOSAA & d3-N-MeFOSAA \\
\hline 3:3 FTCA & M8PFOA \\
\hline 4:3 FTCA & M8PFOA \\
\hline 5:3 FTCA & M8PFOA \\
\hline 7:3 FTCA & M8PFOA \\
\hline 4:2 FTSA & M2-6:2 FTSA \\
\hline 6:2 FTSA & M2-6:2 FTSA \\
\hline 8:2 FTSA & M2-8:2 FTSA \\
\hline 10:2 FTSA & M2-8:2 FTSA \\
\hline 6:2 FTUA & M6:2 FTUA \\
\hline 8:2 FTUA & M8:2 FTUA \\
\hline
\end{tabular}




\begin{tabular}{ll} 
10:2 FTUA & M8:2 FTUA \\
6:2 FTAB & PFOAAmS(N-TAmP-FOAd) \\
5:3 FTB & PFOAAmS(N-TAmP-FOAd) \\
5:1:2 FTB & PFOAAmS(N-TAmP-FOAd) \\
PFHxSAm(AmPr-FHxSA) & PFOAAmS(N-TAmP-FOAd) \\
PFOSAm(AmPr-FOSA) & PFOAAmS(N-TAmP-FOAd) \\
PFHxSAmS(N-TAmP-FHxSA) & PFOAAmS(N-TAmP-FOAd) \\
PFOSAmS(N-TAmP-FOSA) & PFOAAmS(N-TAmP-FOAd) \\
PFOSNO(N-OxAmP-FOSA) & PFOAAmS(N-TAmP-FOAd) \\
PFOANO(N-OxAmP-FOAd) & PFOAAmS(N-TAmP-FOAd) \\
PFOSB(N-CMAmP-FOSA) & PFOAAmS(N-TAmP-FOAd) \\
PFOAB(N-CMAmP-FOAd) & PFOAAmS(N-TAmP-FOAd) \\
\hline
\end{tabular}


Table S9. The acronym, theoretical and observed $\mathrm{m} / \mathrm{z}$, mass error, retention time (RT), analysis method, and identification confidence level of suspect-screening PFAS.

\begin{tabular}{|c|c|c|c|c|c|c|c|c|}
\hline No. & PFAS Class & Acronym & $\begin{array}{l}\text { Theoretical } \\
\mathrm{m} / \mathbf{z}\end{array}$ & $\begin{array}{l}\text { Observed } \\
\mathrm{m} / \mathrm{z}\end{array}$ & $\begin{array}{l}\text { Mass } \\
\text { error, ppm }\end{array}$ & RT, min & $\begin{array}{l}\text { lonization } \\
\text { mode }\end{array}$ & $\begin{array}{l}\text { Identification } \\
\text { confidence level }\end{array}$ \\
\hline 1 & $\mathrm{n}: 2$ FTSA & $14: 2$ FTSA & 826.94230 & 826.94552 & 3.89 & 8.93 & ESI- & Level 4 \\
\hline 2 & $\mathrm{n}: 3$ FTCA & 9:3 FTCA & 540.99141 & 540.99225 & 1.55 & 8.29 & ESI- & Level 4 \\
\hline 3 & $\mathrm{n}: 2$ FTSAS (or FtTAoS) & 6:2 FTSAS & 586.03914 & 586.03912 & -0.03 & 6.08 & ESI- & Level $2 a$ \\
\hline 4 & $\begin{array}{l}\mathrm{n}: 2 \text { FTSAS-SO (or FtSOAoS or } \\
\mathrm{n}: 2 \text { FTSAS-sulfoxide) }\end{array}$ & 6:2 FTSAS-sulfoxide & 602.03457 & 602.03363 & -1.56 & 5.12 & ESI- & Level 2a \\
\hline 5 & $\mathrm{n}: 2$ FTSAS-SO ${ }_{2}$ & 8:2 FTSAS-SO ${ }_{2}$ & 718.02300 & 718.02423 & 1.71 & 6.53 & ESI- & Level $2 a$ \\
\hline 6 & $\mathrm{n}: 2: 1$ FTSC & 4:2:1 FTSC & 336.99448 & 336.99460 & 0.36 & 5.42 & ESI- & Level 4 \\
\hline 7 & $\mathrm{n}: 2$ FASA & 6:2 FASA & 425.98313 & 425.98785 & 11.08 & 6.49 & ESI- & Level 4 \\
\hline 8 & 1HO-n:2 FTS & $1 \mathrm{HO}-6: 2 \mathrm{FTS}$ & 442.96248 & 442.96228 & -0.45 & 4.94 & ESI- & Level 2a \\
\hline 9 & $\mathrm{n}: 2$ FTSO2PA & 8:2 FTSO2PA & 582.98760 & 582.98859 & 1.70 & 7.53 & ESI- & Level 4 \\
\hline 10 & FASAs & FPeSA & 347.95544 & 347.95627 & 2.39 & 6.00 & ESI- & Level 2a \\
\hline 11 & FASAA & FHxSAA & 455.95773 & 455.95905 & 2.90 & 6.52 & ESI- & Level 2a \\
\hline 12 & MeFASA & MeFHxSA & 411.96748 & 411.96936 & 4.56 & 7.30 & ESI- & Level 2a \\
\hline 13 & EtFASA & EtFHxSA & 425.98313 & 425.98523 & 4.93 & 6.49 & ESI- & Level 4 \\
\hline 14 & $\mathrm{~N}$-EtFASAA & N-EtFHxSAA & 483.98861 & 483.99069 & 4.30 & 7.38 & ESI- & Level 2a \\
\hline 15 & N-MeFASAA & N-MeFBSAA & 369.97914 & 369.97934 & 0.54 & 5.44 & ESI- & Level 4 \\
\hline 16 & FASE & FOSE & 441.97867 & 441.97894 & 0.61 & 6.53 & ESI- & Level 4 \\
\hline 17 & N-SP-FASA & N-SP-FHxSA & 519.95612 & 519.95557 & -1.06 & 5.45 & ESI- & Level 4 \\
\hline 18 & PFASi & PFHxSi & 382.94135 & 382.94232 & 2.53 & 5.00 & ESI & Level 4 \\
\hline 19 & n-F5S-PFAS & 8-F5S-PFAS & 606.89587 & 606.89728 & 2.32 & $\begin{array}{l}7.66 \text { (major), } \\
7.45 \text { (minor) }\end{array}$ & ESI- & Level 2a \\
\hline 20 & UPFAS & UPFUnS & 610.92401 & 610.92511 & 1.80 & 7.49 & ESI- & Level $2 a$ \\
\hline 21 & H-UPFAS & H-UPFOS & 442.94260 & 442.94568 & 6.95 & 5.86 & ESI- & Level $2 a$ \\
\hline 22 & H-PFAS & $\mathrm{H}-\mathrm{PFH} \mathrm{xS}$ & 480.93951 & 480.93909 & -0.87 & 5.91 & ESI- & Level 2a \\
\hline 23 & Cl-PFAS (Cl-PFSA) & Cl-PFOS & 514.90064 & 514.90232 & 3.26 & 6.57 & ESI- & Level 2a \\
\hline 24 & O-PFAS (ether-PFSA) & O-PFOS & 514.92490 & 514.92572 & 1.59 & 6.84 & ESI- & Level $2 a$ \\
\hline 25 & ketone-PFSAs & ketone-PFOS & 476.92819 & 476.92780 & -0.82 & 6.27 & ESI- & Level $2 a$ \\
\hline 26 & $\mathrm{n}: 2$ FTAB & $8: 2$ FTAB & 671.08723 & 671.08759 & 0.54 & 6.33 & $\mathrm{ESI}+$ & Level $2 a$ \\
\hline 27 & $\mathrm{n}: 2$ FTA (or FtSaAM, or M4) & $6: 2$ FTA & 513.08814 & 513.08813 & -0.02 & 5.33 & $\mathrm{ESI}+$ & Level 2a \\
\hline 28 & $\mathrm{n}: 2$-demethyl-FTA & $6: 2$ dimethyl-FTA & 499.07249 & 499.07166 & -1.66 & 5.39 & $\mathrm{ESI}+$ & Level 2a \\
\hline 29 & $\mathrm{n}: 3$ FTB & $7: 3$ FTB & 514.08632 & 514.08569 & -1.23 & 5.64 & $\mathrm{ESI}+$ & Level 2a \\
\hline 30 & $\mathrm{n}: 1: 2$ FTB & 7:1:2 FTB & 532.07711 & 532.07587 & -2.33 & 5.71 & $\mathrm{ESI}+$ & Level 2a \\
\hline 31 & $\mathrm{n}: 2$ FTSHA (or FtTHN+) & 6:2 FTSHA & 496.09818 & 496.09723 & -1.91 & 5.70 & $\mathrm{ESI}+$ & Level 2a \\
\hline 32 & $\mathrm{n}: 2$ FTSHA-SO (FtTHN+-SO) & 6:2 FTSHA-SO & 512.09234 & 512.09296 & 1.21 & 4.88 & $\mathrm{ESI}+$ & Level 2a \\
\hline 34 & $\mathrm{n}: 2$ FTSAA & 6:2 FTSAA & 523.10908 & 523.10156 & -14.38 & 5.12 & $\mathrm{ESI}+$ & Level 4 \\
\hline 35 & CMAmEt-FA & $\begin{array}{l}\text { 6CMAmEt-FA (or } 6: 2 \\
\text { FTB) }\end{array}$ & 450.07387 & 450.07367 & -0.44 & 4.76 & $\mathrm{ESI+}$ & Level 2a \\
\hline 36 & CMAmB-FA & $\begin{array}{l}\text { 6CMAmB-FA (or } 6: 4 \\
\text { FTB) }\end{array}$ & 478.10496 & 478.10477 & -0.40 & 5.47 & $\mathrm{ESI}+$ & Level 2a \\
\hline
\end{tabular}




\begin{tabular}{|c|c|c|c|c|c|c|c|c|}
\hline 37 & $\begin{array}{l}\text { AmPr-FASAP (PFASAC, or } \\
\text { PFnSAmA) }\end{array}$ & AmPr-FHxSAP & 557.07828 & 557.07776 & -0.93 & 5.37 & ESI+ & Level 2a \\
\hline 38 & $\begin{array}{l}\text { N-CEAmP-FASAP (PFASBC or } \\
\text { PFASA2C) }\end{array}$ & $\mathrm{N}$-CEAmP-FPeSAP & 579.10250 & 579.10175 & -1.30 & 6.46 & ESI+ & Level 2a \\
\hline 39 & N-CMAmP-FAAd (PFAAB) & N-CMAmP-FHpAd & 507.09668 & 507.09134 & -10.53 & 5.45 & ESI+ & Level 4 \\
\hline 40 & N-CMAmP-FASA (PFASB) & N-CMAmP-FHxSA & 543.06211 & 543.06165 & -0.85 & $\begin{array}{l}5.34 \text { (major) } \\
5.19 \text { (minor) }\end{array}$ & ESI+ & Level 2a \\
\hline 41 & N-TAmP-FASA (PFASAmS) & N-TAmP-FPeSA & 449.07537 & 449.07535 & -0.04 & 4.87 & ESI+ & Level $2 a$ \\
\hline 42 & AmPr-FASA (PFASAm) & AmPr-FPeSA & 435.05972 & 435.05966 & -0.14 & $\begin{array}{l}4.84 \text { (major), } \\
4.70 \text { (minor) }\end{array}$ & $\mathrm{ESI}+$ & Level $2 a$ \\
\hline 43 & AmPr-FAAd (PFAAAm) & AmPr-FHxAd & 399.09325 & 399.09314 & -0.28 & 4.18 & ESI+ & Level $2 \mathrm{a}$ \\
\hline 44 & N-OxAmP-FASA (PFASNO) & N-OxAmP-FHxSA & 501.05155 & 501.05096 & -1.18 & 5.42 & ESI+ & Level $2 a$ \\
\hline 45 & N-SPAmP-FASAA & N-SPAmP-FHxSAA & 663.05075 & 663.05072 & -0.05 & 5.35 & ESI+ & Level 4 \\
\hline 46 & N-SPAmP-FASA & N-SPAmP-FHxSA & 607.06040 & 607.05963 & -1.27 & 5.18 & ESI+ & Level $2 a$ \\
\hline 47 & N-SPHOEAmP-FASA & N-SPHOEAmP-FBSA & 537.07756 & 537.07892 & 2.53 & 5.50 & ESI+ & Level $2 a$ \\
\hline 48 & N-HOEAmP-FASAPS & N-HOEAmP-FHxSAPS & 651.08724 & 651.08661 & -0.97 & 5.07 & ESI+ & Level $2 \mathrm{a}$ \\
\hline 49 & N-HOEAmP-FASA & N-HOEAmP-FHxSA & 529.08316 & 529.08234 & -1.55 & 5.28 & ESI+ & Leve $2 a$ \\
\hline 50 & N-HOEAmP-FASE & N-HOEAmP-FHxSE & 573.10968 & 573.10870 & -1.71 & $\begin{array}{l}5.25 \text { (major) } \\
5.11 \text { (minor) }\end{array}$ & ESI+ & Level 2a \\
\hline 51 & N-HOEAmHOP-FASA & N-HOEAmHOP-FHxSA & 545.07818 & 545.07794 & -0.44 & $\begin{array}{l}5.18 \text { (major) } \\
5.07 \text { (minor) }\end{array}$ & ESI+ & Level 2a \\
\hline 52 & N-SPAmP-FASAPS & N-SPAmP-FBSAPS & 629.07087 & 629.06647 & -6.99 & 4.96 & ESI+ & Level 4 \\
\hline 53 & N-TamP-N-MeFASA & N-TamP-N-MeFHxSA & 513.08835 & 513.08698 & -2.67 & $\begin{array}{l}5.58 \text { (major) } \\
5.32 \text { (minor) }\end{array}$ & ESI+ & Level 2a \\
\hline 54 & N-TAmP-FASAP & N-TAmP-FHxSAP & 571.09393 & 571.09344 & -0.86 & 5.38 & ESI+ & Level 2a \\
\hline 55 & N-CMAmP-FASAP & N-CMAmP-FBSAP & 515.08963 & 515.08893 & -1.36 & 5.66 & ESI+ & Level 2a \\
\hline 56 & N-CMAmP-FASAA & N-CMAmP-FPrSAA & 451.07738 & 451.07724 & -0.31 & 4.76 & ESI+ & Level 2a \\
\hline 57 & $\mathrm{~N}$-CEAmP-EtFASA & $\mathrm{N}$-CEAmP-EtFHxSA & 585.10937 & 585.10944 & 0.12 & 5.58 & ESI+ & Level 2a \\
\hline
\end{tabular}


Table S10. Compound-specific instrumental limits of detection (iLOD), instrumental limits of quantification (iLOQ), method limits of detection ( $\mathrm{mLOD}$ ), and limits of quantification ( $\mathrm{mLOQ}$ ) and linearity performance of 53 quantitative PFAS in soil and groundwater.

(a) In soil. The accuracy and precision performance of QC samples inserted along the analytical sequence at a medium spike level $(5 \mathrm{ng} / \mathrm{mL})$

\begin{tabular}{|c|c|c|c|c|c|c|c|c|}
\hline Analyte & Linearity range & $\mathrm{R}^{2}$ & $\begin{array}{l}\text { iLOD } \\
(\mu g / L)\end{array}$ & $\begin{array}{l}\text { iLOQ } \\
(\mu \mathrm{g} / \mathrm{L})\end{array}$ & $\begin{array}{l}\text { mLOD } \\
(\mu \mathrm{g} / \mathrm{kg} \mathrm{dw})\end{array}$ & $\begin{array}{l}\mathrm{mLOQ} \\
(\mu \mathrm{g} / \mathrm{kg} \mathrm{dw})\end{array}$ & $\begin{array}{l}\text { Accuracy }(\%) \\
\text { at } 5 \mathrm{ng} / \mathrm{mL} n=5, \\
\text { (Average } \pm S D)\end{array}$ & $\begin{array}{l}\text { Precision } \\
N=5 \\
(\mathrm{RSD}, \%)\end{array}$ \\
\hline PFPrA & $0.2-25$ & 0.9984 & 0.090 & 0.200 & 0.180 & 0.400 & $98 \pm 5$ & 5.2 \\
\hline PFBA & $0.2-25$ & 0.9989 & 0.080 & 0.200 & 0.160 & 0.400 & $97 \pm 2$ & 1.8 \\
\hline PFPeA & $0.05-25$ & 0.9992 & 0.020 & 0.050 & 0.040 & 0.100 & $96 \pm 2$ & 1.6 \\
\hline PFHxA & $0.025-25$ & 0.9989 & 0.030 & 0.025 & 0.060 & 0.050 & $96 \pm 3$ & 2.5 \\
\hline PFHpA & $0.025-25$ & 0.9994 & 0.007 & 0.025 & 0.014 & 0.050 & $97 \pm 1$ & 1.1 \\
\hline PFOA & $0.025-25$ & 0.9985 & 0.009 & 0.025 & 0.018 & 0.050 & $96 \pm 2$ & 1.6 \\
\hline PFNA & $0.025-25$ & 0.9992 & 0.010 & 0.025 & 0.020 & 0.050 & $96 \pm 1$ & 1.0 \\
\hline PFDA & $0.025-25$ & 0.9991 & 0.010 & 0.025 & 0.020 & 0.050 & $95 \pm 1$ & 0.6 \\
\hline PFUnA & $0.025-25$ & 0.9992 & 0.020 & 0.025 & 0.040 & 0.050 & $96 \pm 1$ & 0.8 \\
\hline PFDoA & $0.05-25$ & 0.9992 & 0.030 & 0.050 & 0.060 & 0.100 & $97 \pm 1$ & 1.3 \\
\hline PFTrDA & $0.05-25$ & 0.9986 & 0.030 & 0.050 & 0.060 & 0.100 & $94 \pm 3$ & 3.2 \\
\hline PFTeDA & $0.05-25$ & 0.9992 & 0.030 & 0.050 & 0.060 & 0.100 & $98 \pm 2$ & 1.6 \\
\hline PFHxDA & $0.05-25$ & 0.9970 & 0.030 & 0.050 & 0.060 & 0.100 & $99 \pm 4$ & 4.2 \\
\hline PFPrS & $0.025-25$ & 0.9999 & 0.005 & 0.025 & 0.010 & 0.050 & $93 \pm 4$ & 3.8 \\
\hline PFBS & $0.025-25$ & 0.9994 & 0.007 & 0.025 & 0.014 & 0.050 & $95 \pm 1$ & 0.9 \\
\hline PFPeS & $0.025-25$ & 0.9977 & 0.005 & 0.025 & 0.010 & 0.050 & $98 \pm 3$ & 2.7 \\
\hline PFHxS & $0.025-25$ & 0.9992 & 0.006 & 0.025 & 0.012 & 0.050 & $94 \pm 1$ & 0.6 \\
\hline PFHpS & $0.025-25$ & 0.9967 & 0.006 & 0.025 & 0.012 & 0.050 & $95 \pm 4$ & 3.7 \\
\hline PFOS & $0.025-25$ & 0.9990 & 0.020 & 0.025 & 0.040 & 0.050 & $96 \pm 1$ & 0.8 \\
\hline PFNS & $0.025-25$ & 0.9992 & 0.007 & 0.025 & 0.014 & 0.050 & $100 \pm 3$ & 3.3 \\
\hline PFDS & $0.025-25$ & 0.9991 & 0.005 & 0.025 & 0.010 & 0.050 & $95 \pm 3$ & 3.1 \\
\hline PFDoS & $0.05-25$ & 0.9994 & 0.010 & 0.050 & 0.020 & 0.100 & $102 \pm 9$ & 8.6 \\
\hline 4:2 FTSA & $0.025-25$ & 0.9986 & 0.005 & 0.025 & 0.010 & 0.050 & $98 \pm 2$ & 2.2 \\
\hline 6:2 FTSA & $0.05-25$ & 0.9928 & 0.040 & 0.050 & 0.080 & 0.100 & $116 \pm 12$ & 12.4 \\
\hline 8:2 FTSA & $0.025-25$ & 0.9988 & 0.010 & 0.025 & 0.020 & 0.050 & $96 \pm 2$ & 1.7 \\
\hline 10:2 FTSA & $0.05-25$ & 0.9996 & 0.020 & 0.050 & 0.040 & 0.100 & $96 \pm 3$ & 2.6 \\
\hline 3:3 FTCA & $0.2-25$ & 0.9987 & 0.050 & 0.200 & 0.100 & 0.400 & $93 \pm 6$ & 5.9 \\
\hline 4:3 FTCA & $0.1-25$ & 0.9975 & 0.050 & 0.100 & 0.100 & 0.200 & $94 \pm 3$ & 2.8 \\
\hline 5:3 FTCA & $0.1-25$ & 0.9969 & 0.050 & 0.100 & 0.100 & 0.200 & $94 \pm 3$ & 3.5 \\
\hline 7:3 FTCA & $0.1-25$ & 0.9990 & 0.030 & 0.100 & 0.060 & 0.200 & $97 \pm 4$ & 4.1 \\
\hline 6:2 FTUA & $0.1-25$ & 0.9986 & 0.100 & 0.100 & 0.200 & 0.200 & $100 \pm 1$ & 1.4 \\
\hline 8:2 FTUA & $0.05-25$ & 0.9987 & 0.050 & 0.050 & 0.100 & 0.100 & $98 \pm 1$ & 1.3 \\
\hline 10:2 FTUA & $0.05-25$ & 0.9970 & 0.050 & 0.050 & 0.100 & 0.100 & $103 \pm 3$ & 3.4 \\
\hline 6:2 FTAB & $0.05-25$ & 0.9966 & 0.040 & 0.050 & 0.080 & 0.100 & $95 \pm 7$ & 6.6 \\
\hline 5:3 FTB & $0.05-25$ & 0.9992 & 0.040 & 0.050 & 0.080 & 0.100 & $119 \pm 12$ & 11.6 \\
\hline 5:1:2 FTB & $0.05-25$ & 0.9998 & 0.040 & 0.050 & 0.080 & 0.100 & $92 \pm 2$ & 2.1 \\
\hline FBSA & $0.025-10$ & 0.9935 & 0.008 & 0.025 & 0.016 & 0.050 & $99 \pm 7$ & 6.9 \\
\hline FHxSA & $0.025-25$ & 0.9979 & 0.009 & 0.025 & 0.018 & 0.050 & $101 \pm 5$ & 4.9 \\
\hline FOSA & $0.025-25$ & 0.9992 & 0.010 & 0.025 & 0.020 & 0.050 & $97 \pm 1$ & 0.9 \\
\hline MeFOSA & $0.025-25$ & 0.9922 & 0.010 & 0.025 & 0.020 & 0.050 & $87 \pm 5$ & 4.7 \\
\hline EtFOSA & $0.025-25$ & 0.9978 & 0.010 & 0.025 & 0.020 & 0.050 & $99 \pm 6$ & 5.6 \\
\hline FOSAA & $0.05-25$ & 0.9978 & 0.020 & 0.050 & 0.040 & 0.100 & $96 \pm 3$ & 2.8 \\
\hline MeFOSAA & $0.1-25$ & 0.9955 & 0.070 & 0.100 & 0.140 & 0.200 & $113 \pm 16$ & 16.4 \\
\hline EtFOSAA & $0.1-25$ & 0.9906 & 0.060 & 0.100 & 0.120 & 0.200 & $93 \pm 9$ & 9.3 \\
\hline PFHxSAm & $0.05-25$ & 0.9991 & 0.010 & 0.050 & 0.020 & 0.100 & $88 \pm 7$ & 6.7 \\
\hline PFOSAm & $0.05-25$ & 0.9990 & 0.010 & 0.050 & 0.020 & 0.100 & $90 \pm 6$ & 6.0 \\
\hline PFHxSAmS & $0.05-25$ & 0.9967 & 0.020 & 0.050 & 0.040 & 0.100 & $82 \pm 5$ & 4.6 \\
\hline PFOSAmS & $0.05-25$ & 0.9972 & 0.020 & 0.050 & 0.040 & 0.100 & $80 \pm 5$ & 4.7 \\
\hline PFOAB & $0.1-25$ & 0.9977 & 0.080 & 0.100 & 0.160 & 0.200 & $94 \pm 4$ & 4.0 \\
\hline PFOSB & $0.2-25$ & 0.9981 & 0.070 & 0.200 & 0.140 & 0.400 & $92 \pm 3$ & 2.6 \\
\hline PFOANO & $0.05-25$ & 0.9988 & 0.030 & 0.050 & 0.060 & 0.100 & $90 \pm 8$ & 7.8 \\
\hline PFOSNO & $0.05-25$ & 0.9975 & 0.007 & 0.050 & 0.014 & 0.100 & $90 \pm 4$ & 3.9 \\
\hline PFECHS & $0.025-25$ & 0.9986 & 0.005 & 0.025 & 0.010 & 0.050 & $91 \pm 2$ & 1.8 \\
\hline
\end{tabular}


(b) In groundwater. The volume of $\mathrm{MeOH}$ and HPLC grade-water was 80/20 (v/v) when building the matrixfree solvent calibration curve.

\begin{tabular}{|c|c|c|c|c|c|c|}
\hline Analyte & Linearity range & $\mathrm{R}^{2}$ & $\begin{array}{l}\text { iLOD } \\
(\mu \mathrm{g} / \mathrm{L})\end{array}$ & $\begin{array}{l}\text { iLOQ } \\
(\mu \mathrm{g} / \mathrm{L})\end{array}$ & $\begin{array}{l}\mathrm{mLOD} \\
(\mu \mathrm{g} / \mathrm{L})\end{array}$ & $\begin{array}{l}\mathrm{mLOQ} \\
(\mu \mathrm{g} / \mathrm{L})\end{array}$ \\
\hline PFPrA & $0.05-25$ & 0.9998 & 0.025 & 0.050 & 0.210 & 0.420 \\
\hline PFBA & $0.05-26$ & 0.9998 & 0.014 & 0.050 & 0.118 & 0.420 \\
\hline PFPeA & $0.01-25$ & 0.9998 & 0.004 & 0.010 & 0.034 & 0.084 \\
\hline PFHXA & $0.01-25$ & 0.9998 & 0.004 & 0.010 & 0.034 & 0.084 \\
\hline PFHpA & $0.01-25$ & 0.9997 & 0.005 & 0.010 & 0.042 & 0.084 \\
\hline PFOA & $0.01-25$ & 0.9997 & 0.006 & 0.010 & 0.050 & 0.084 \\
\hline PFNA & $0.01-25$ & 0.9995 & 0.005 & 0.010 & 0.042 & 0.084 \\
\hline PFDA & $0.01-25$ & 0.9998 & 0.006 & 0.010 & 0.050 & 0.084 \\
\hline PFUnA & $0.025-25$ & 0.9997 & 0.007 & 0.025 & 0.059 & 0.210 \\
\hline PFDoA & $0.05-25$ & 0.9992 & 0.010 & 0.050 & 0.084 & 0.420 \\
\hline PFTrDA & $0.05-25$ & 0.9986 & 0.010 & 0.050 & 0.084 & 0.420 \\
\hline PFTeDA & $0.05-25$ & 0.9992 & 0.010 & 0.050 & 0.084 & 0.420 \\
\hline PFHxDA & $0.05-25$ & 0.9970 & 0.010 & 0.050 & 0.084 & 0.420 \\
\hline PFPrS & $0.1-25$ & 0.9998 & 0.050 & 0.100 & 0.420 & 0.840 \\
\hline PFBS & $0.01-25$ & 0.9996 & 0.008 & 0.010 & 0.067 & 0.084 \\
\hline PFPeS & $0.01-25$ & 0.9997 & 0.005 & 0.010 & 0.042 & 0.084 \\
\hline PFHxS & $0.01-25$ & 0.9998 & 0.005 & 0.010 & 0.042 & 0.084 \\
\hline PFHpS & $0.01-25$ & 0.9994 & 0.002 & 0.010 & 0.017 & 0.084 \\
\hline PFOS & $0.01-25$ & 0.9995 & 0.002 & 0.010 & 0.017 & 0.084 \\
\hline PFNS & $0.01-25$ & 0.9999 & 0.002 & 0.010 & 0.017 & 0.084 \\
\hline PFDS & $0.01-25$ & 1.0000 & 0.004 & 0.010 & 0.034 & 0.084 \\
\hline PFDoS & $0.01-25$ & 0.9994 & 0.005 & 0.010 & 0.042 & 0.084 \\
\hline $4: 2$ FTSA & $0.025-25$ & 0.9999 & 0.006 & 0.025 & 0.050 & 0.210 \\
\hline 6:2 FTSA & $0.025-25$ & 0.9999 & 0.012 & 0.025 & 0.101 & 0.210 \\
\hline 8:2 FTSA & $0.025-25$ & 0.9993 & 0.013 & 0.025 & 0.109 & 0.210 \\
\hline 10:2 FTSA & $0.05-25$ & 0.9991 & 0.026 & 0.050 & 0.218 & 0.420 \\
\hline 3:3 FTCA & $0.1-25$ & 0.9987 & 0.050 & 0.100 & 0.420 & 0.840 \\
\hline $4: 3$ FTCA & $0.1-25$ & 0.9975 & 0.050 & 0.100 & 0.420 & 0.840 \\
\hline 5:3 FTCA & $0.1-25$ & 0.9999 & 0.020 & 0.100 & 0.168 & 0.840 \\
\hline 7:3 FTCA & $0.1-25$ & 0.9996 & 0.075 & 0.100 & 0.630 & 0.840 \\
\hline 6:2 FTUA & $0.25-25$ & 0.9989 & 0.008 & 0.250 & 0.067 & 2.100 \\
\hline 8:2 FTUA & $0.05-25$ & 0.9980 & 0.006 & 0.050 & 0.050 & 0.420 \\
\hline 10:2 FTUA & $0.025-25$ & 0.9979 & 0.006 & 0.025 & 0.050 & 0.210 \\
\hline FBSA & $0.01-25$ & 0.9999 & 0.002 & 0.010 & 0.017 & 0.084 \\
\hline FHxSA & $0.01-25$ & 0.9999 & 0.002 & 0.010 & 0.017 & 0.084 \\
\hline FOSA & $0.01-25$ & 0.9998 & 0.005 & 0.010 & 0.042 & 0.084 \\
\hline MeFOSA & $0.01-25$ & 0.9999 & 0.002 & 0.010 & 0.017 & 0.084 \\
\hline EtFOSA & $0.025-25$ & 0.9998 & 0.008 & 0.025 & 0.067 & 0.210 \\
\hline FOSAA & $0.05-25$ & 0.9995 & 0.012 & 0.050 & 0.101 & 0.420 \\
\hline MeFOSAA & $0.05-25$ & 0.9999 & 0.025 & 0.050 & 0.210 & 0.420 \\
\hline EtFOSAA & $0.05-25$ & 0.9999 & 0.007 & 0.050 & 0.059 & 0.420 \\
\hline PFHxSAm & $0.05-25$ & 0.9996 & 0.007 & 0.050 & 0.059 & 0.420 \\
\hline PFOSAm & $0.05-25$ & 0.9996 & 0.007 & 0.050 & 0.059 & 0.420 \\
\hline PFHxSAmS & $0.025-25$ & 0.9995 & 0.014 & 0.025 & 0.118 & 0.210 \\
\hline PFOSAmS & $0.025-25$ & 0.9995 & 0.014 & 0.025 & 0.118 & 0.210 \\
\hline PFOAB & $0.05-25$ & 0.9977 & 0.080 & 0.050 & 0.672 & 0.420 \\
\hline PFOSB & $0.1-25$ & 0.9981 & 0.070 & 0.100 & 0.588 & 0.840 \\
\hline PFOANO & $0.05-25$ & 0.9988 & 0.030 & 0.050 & 0.252 & 0.420 \\
\hline PFOSNO & $0.05-25$ & 0.9975 & 0.007 & 0.050 & 0.059 & 0.420 \\
\hline $6: 2$ FTAB & $0.05-25$ & 0.9966 & 0.040 & 0.050 & 0.336 & 0.420 \\
\hline $5: 3$ FTB & $0.05-50$ & 0.9992 & 0.020 & 0.050 & 0.168 & 0.420 \\
\hline $5: 1: 2$ FTB & $0.05-50$ & 0.9998 & 0.020 & 0.050 & 0.168 & 0.420 \\
\hline PFECHS & $0.01-25$ & 1.0000 & 0.002 & 0.010 & 0.0168 & 0.084 \\
\hline
\end{tabular}


Table S11. The median PFHxS/PFOS ratio in surface soil and groundwater at different areas of four FTA sites.

\begin{tabular}{|c|c|c|c|}
\hline $\begin{array}{l}\text { Sample } \\
\text { type }\end{array}$ & Site name & Area & PFHxS/PFOS (median) \\
\hline \multirow{6}{*}{ Surface soil } & \multirow{3}{*}{ Site \#1 } & Upgradient & 0.17 \\
\hline & & Vicinity & 0.01 \\
\hline & & Downgradient & 0.05 \\
\hline & Site \#2 & Vicinity & 0.04 \\
\hline & Site \#3 & Vicinity & 0.07 \\
\hline & Site \#4 & Vicinity & 0.09 \\
\hline \multirow{10}{*}{ Groundwater } & \multirow{3}{*}{ Site \#1 } & Upgradient & 0.14 \\
\hline & & Vicinity & 0.20 \\
\hline & & Downgradient & 1.04 \\
\hline & \multirow{3}{*}{ Site \#2 } & Upgradient & $\mathrm{NA}^{*}$ \\
\hline & & Vicinity & 0.19 \\
\hline & & Downgradient & 0.81 \\
\hline & Site \#3 & Vicinity & 0.35 \\
\hline & \multirow{3}{*}{ Site\#4 } & Upgradient & 0.33 \\
\hline & & Vicinity & 0.19 \\
\hline & & Downgradient & 0.13 \\
\hline
\end{tabular}

The red $\operatorname{star}\left({ }^{*}\right):$ The PFOS concentrations in the upgradient groundwater samples at Site \#2 were nondetectable thus the PFHxS/PFOS ratios were not available for those samples. 
Table S12. A draft priority PFAS analyte list for surface soil (a) and groundwater (b).

(a) PFAS analyte priority list for surface soil

\begin{tabular}{ll}
\hline No. & PFAS Analyte \\
\hline 1 & PFOS \\
2 & $7: 1: 2$ FTB \\
3 & $8: 2$ FTSA \\
4 & $6: 2$ FTAB \\
5 & $5: 1: 2$ FTB \\
6 & $6: 2$ FTSA \\
7 & $6: 2$ demethyl-FTA \\
8 & N-TAmP-FHxSA \\
9 & $6: 2$ FTSHA-SO \\
10 & N-HOEAmP-FHxSA \\
11 & PFHxS \\
12 & $7: 3$ FTB \\
13 & $5: 3$ FTB \\
14 & $9: 1: 2$ FTB \\
15 & N-CMAmP-FBSAP \\
16 & FHxSA \\
17 & $11: 1: 2$ FTB \\
18 & PFHpS \\
19 & PFHxA \\
20 & $10: 2$ FTSA \\
21 & $6: 2$ FTA \\
22 & AmPr-FHxSA \\
23 & $8: 2$ FTSO $P A$ \\
24 & PFNS \\
25 & N-CMAmP-FHxSA \\
\hline
\end{tabular}

(b) PFAS analyte priority list for groundwater

\begin{tabular}{ll}
\hline No. & PFAS Analyte \\
\hline 1 & $6: 2$ FTSA \\
2 & FBSA \\
3 & FHxSA \\
4 & PFOS \\
5 & FPeSA \\
6 & $6: 2$ FTSAS-SO \\
7 & PFHxS \\
8 & FPrSA \\
9 & $6: 2$ FTSAS-SO \\
10 & PFHxA \\
11 & PFPeA \\
12 & MeFBSAA \\
13 & PFOA \\
14 & PFBA \\
15 & PFHpA \\
16 & $8: 2$ FTSA \\
17 & N-CMAmP-FHxSA \\
18 & N-SPAmP-FHxSA \\
19 & $8: 2$ FTSAS-SO \\
20 & $4: 2$ FTA \\
21 & PFPeS \\
22 & N-TamP-N-MeFBSA \\
23 & AmPr-FHxSAP \\
24 & N-SP-FHxSA \\
25 & PFHpS \\
\hline
\end{tabular}


Figure S1. Sampling locations for soil and groundwater samples from the four FTA sites, and site history. Note: $\otimes$ soil samples, $\square$ groundwater samples, $\square$ Groundwater flow direction, and $\Longrightarrow$ prevailing wind direction. The red line represents the FTA area boundary.

(a) Site \#1. (Green line - decommissioned FTA; pink line - active FTA)

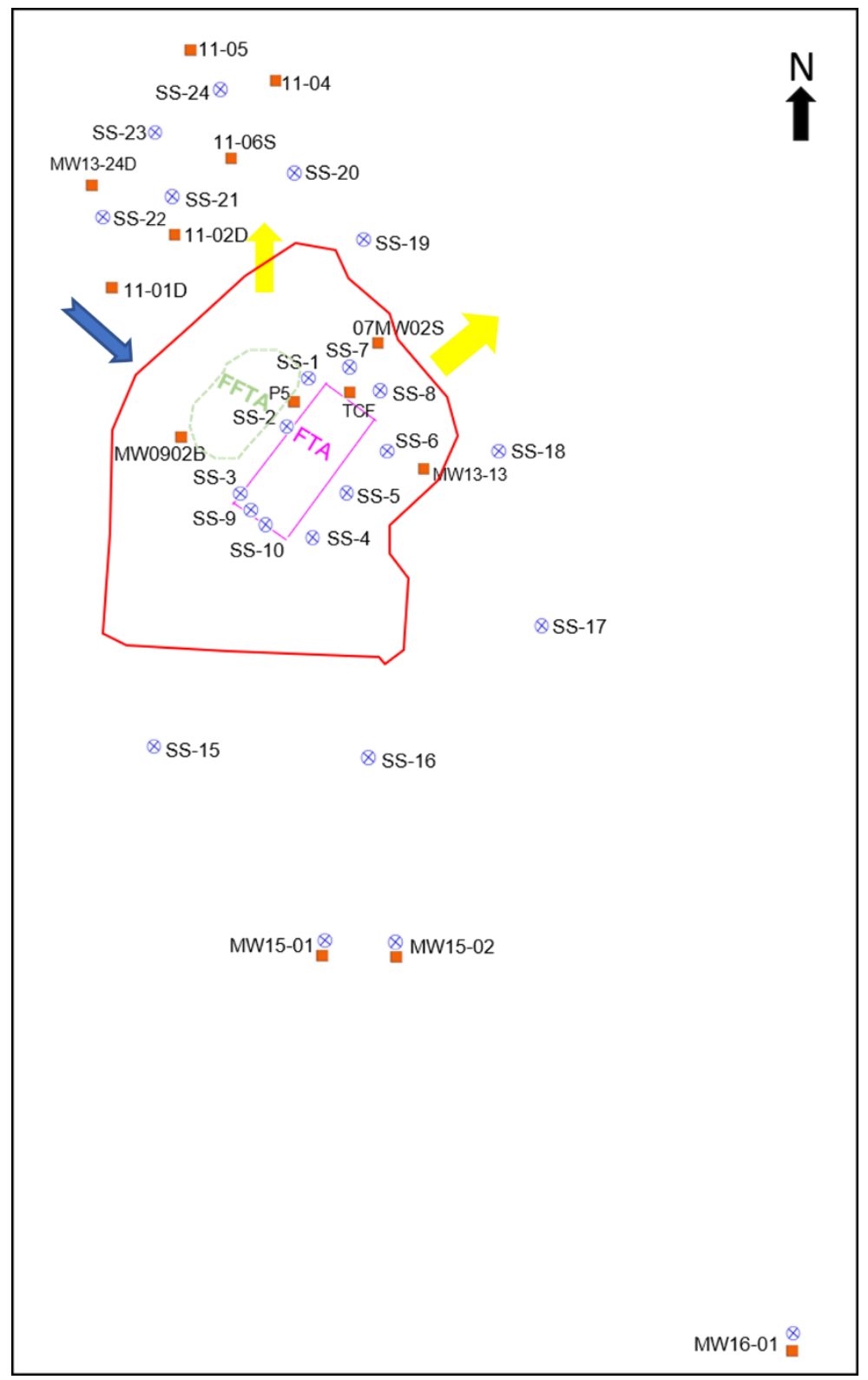


(b) Site \#2 (Decommissioned FTA)

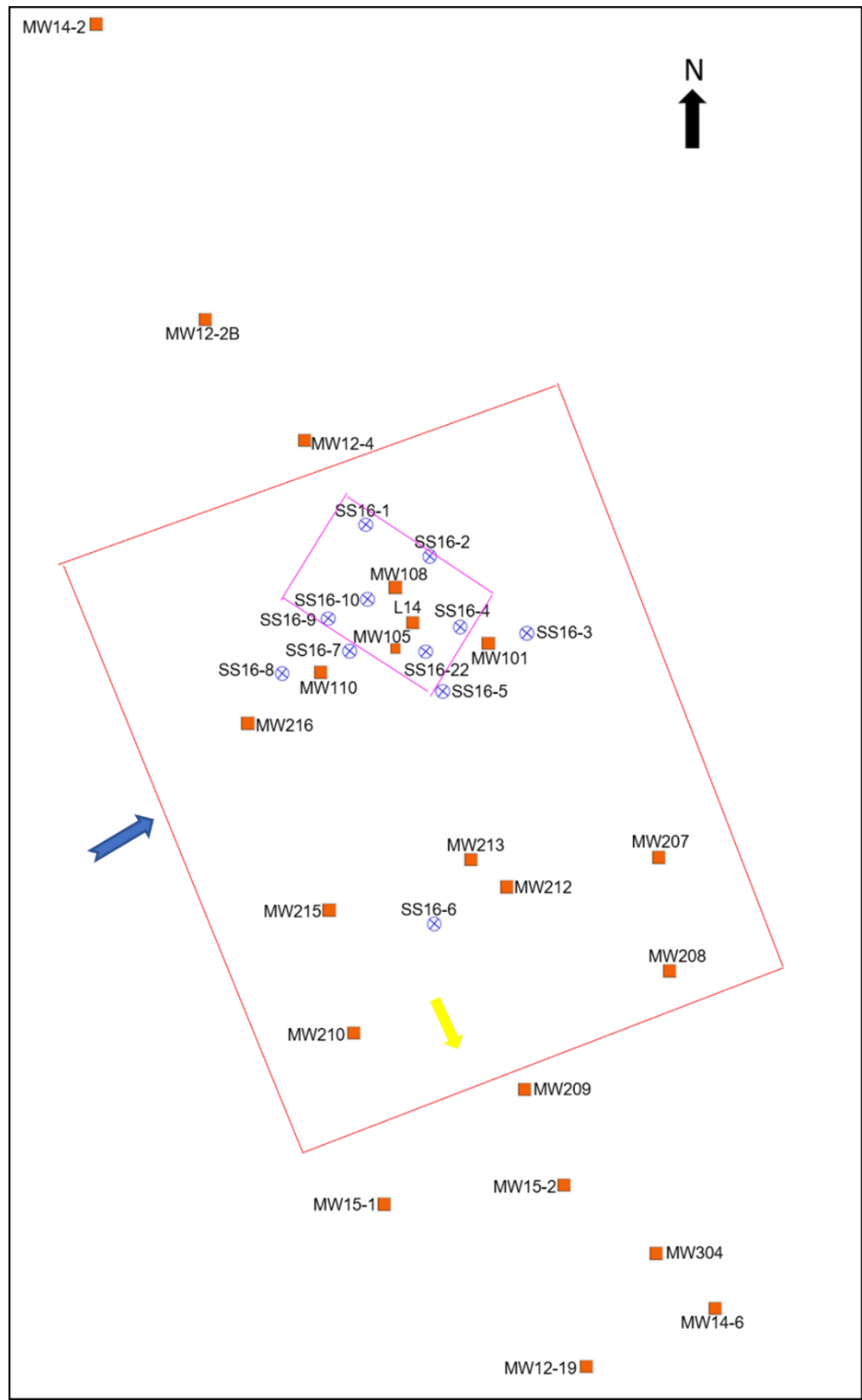


(c) Site \#3 (Decommissioned FTA).

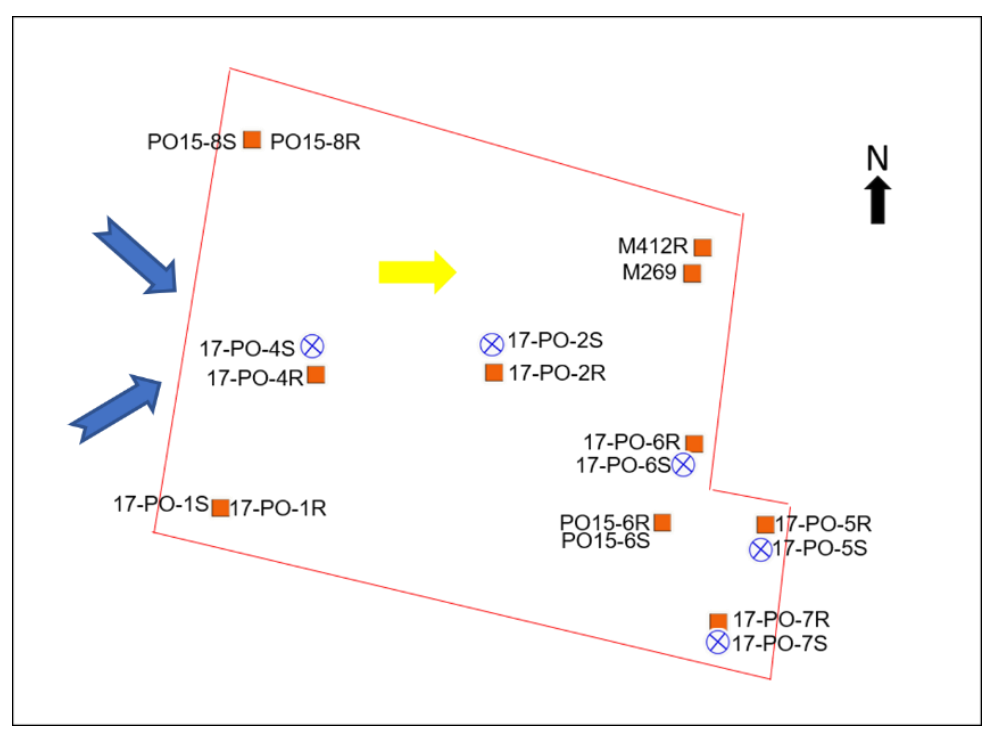

(d) Site \#4 (Decommissioned FTA).

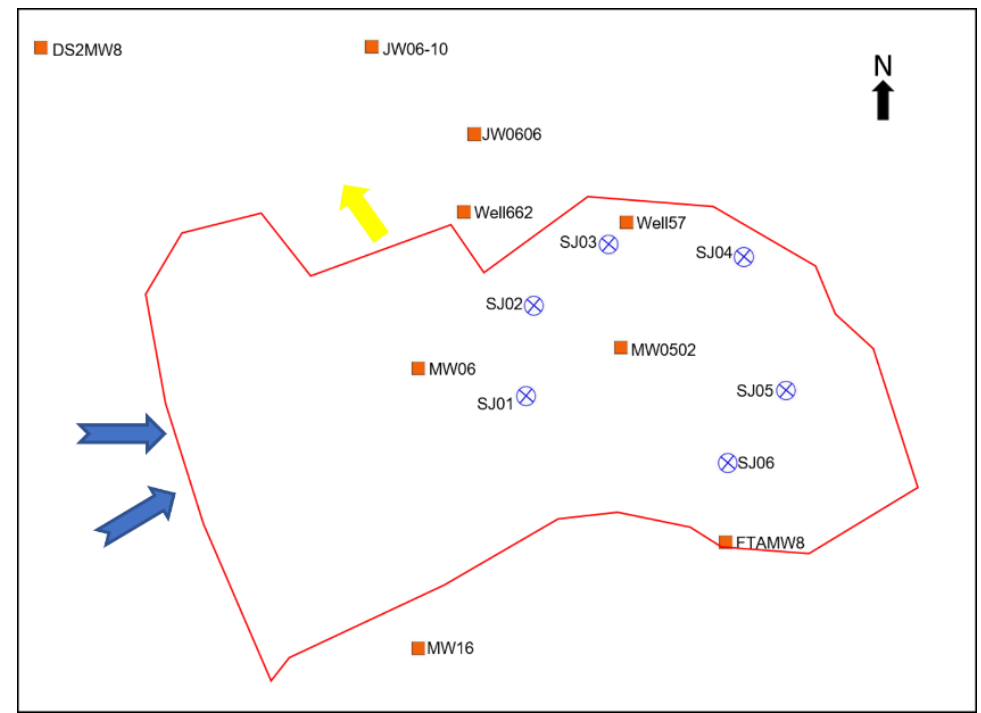

(e) The periods of active fire training for the four Canadian FTA sites: Site \#1 included a former FTA area (FFTA) and an active FTA area (FTA). The field soil and groundwater samples were sampled between Sep. 2016 and Feb. 2017.

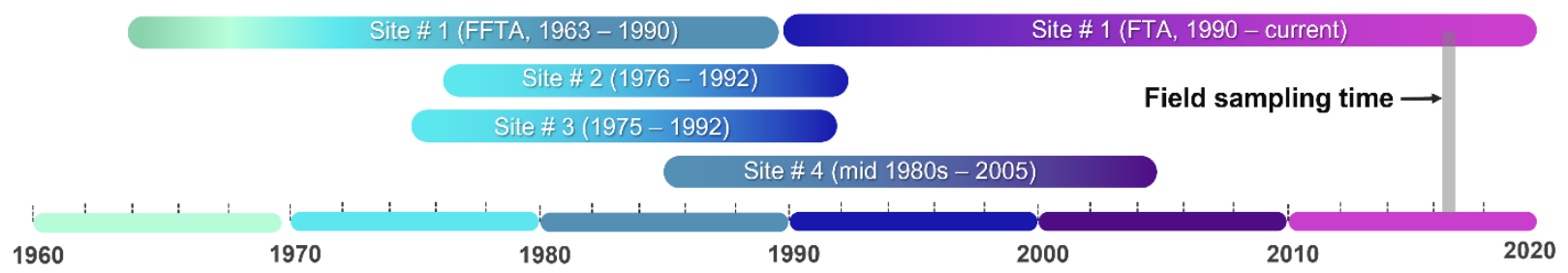


Figure S2. Recovery of PFAS during the freeze-drying step in the soil matrix. Error bars represent standard deviations.

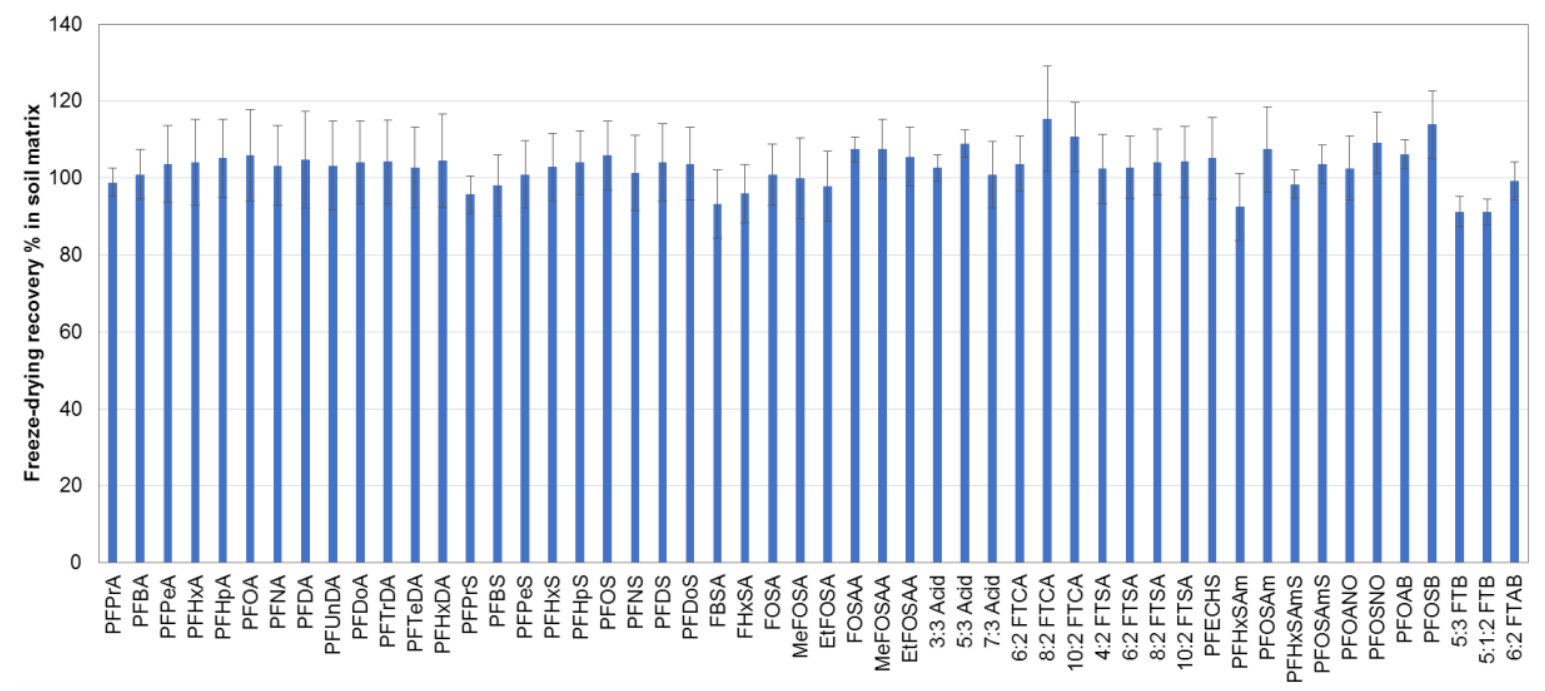


Figure S3. The PFAA recovery in three types of soils during the whole TOP procedure.

Note: Sixty-seven microliters of 1.79 ppm of PFCA (C3-C9) and PFSA (C4, C6, C7, and C8) were spiked into $1 \mathrm{~g}$-dw soil, which then underwent extraction, ENVI-Carb cleanup, nitrogen evaporation to dryness, and the TOP procedure before instrument analysis. PFAAs do not undergo degradation during the TOP assay.

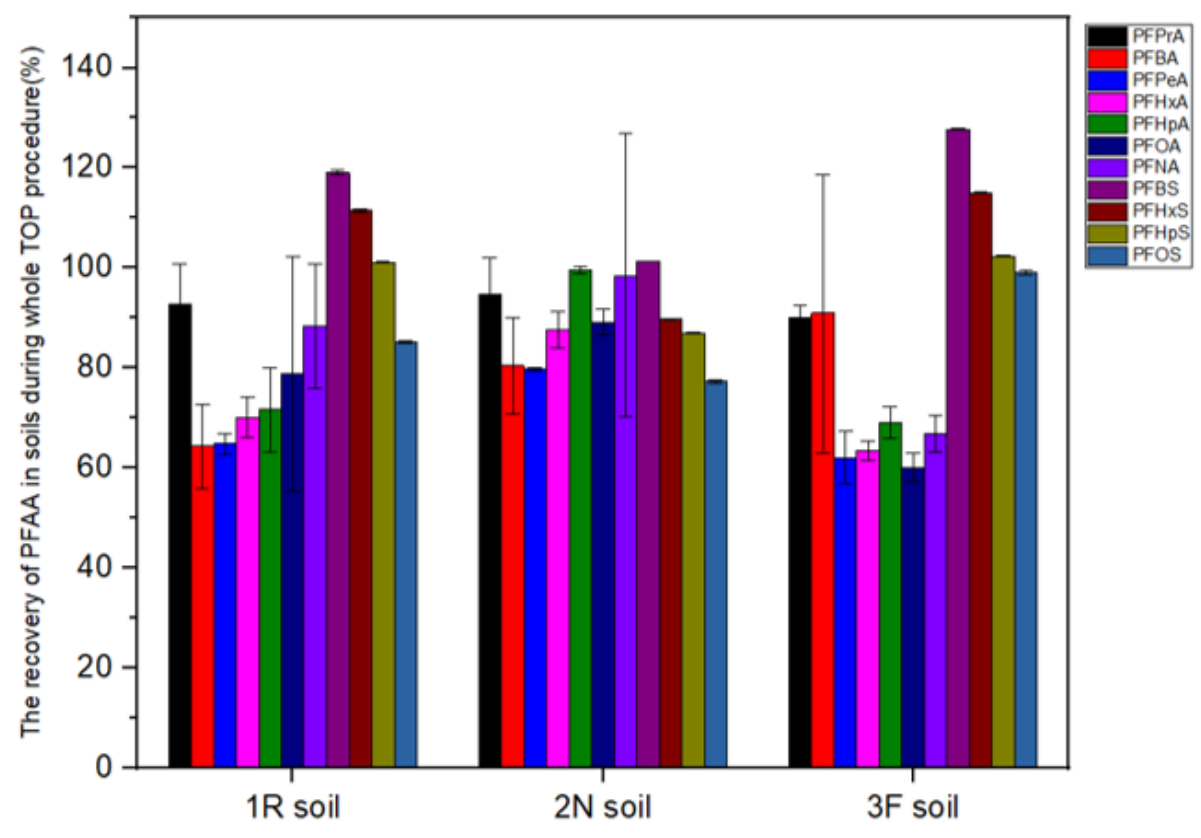


Figure S4. The molar conversion yields of five FT-based and three ECF-based precursors into C3-C10 PFCA post TOP assay in three types of soil matrixes (1R, $2 \mathrm{~N}$ and $3 \mathrm{~F}$ soil) and ultra-pure water; the precursors included 6:2 FTSA (a), 8:2 FTSA (b), 6:2 FTAB (c), 5:3 FTB (d), 5:1:2 FTB (e), FHxSA (f), PFHxSAm (g), and PFHxSAmS (h).
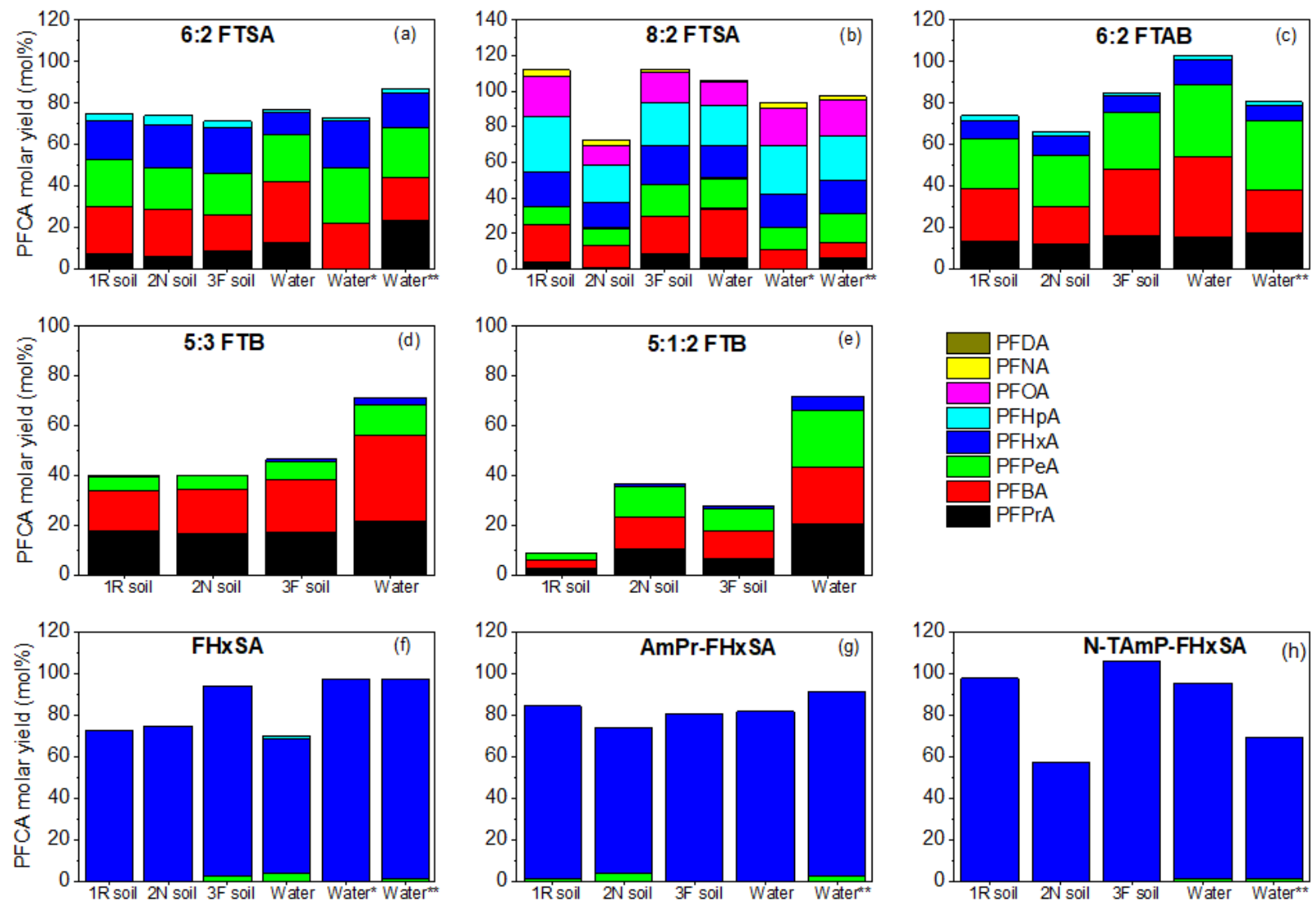

Note: The oxidation conditions, including oxidant concentration and reaction time, were selected from the literature ${ }^{5}$. Sixty-seven $\mu \mathrm{l}$ of $179 \mathrm{ppm}$ stock solution of each precursor was spiked into $1 \mathrm{~g}$-dw soil matrixes, which then underwent extraction, Envi-carb cleanup, nitrogen evaporation to dryness, and TOP assay procedure before instrument analysis. Three replicates were executed per treatment condition. The asterisk indicates oxidation data from Houtz et al,${ }^{5}$ and the double-asterisk $\left({ }^{* *}\right)$ represent oxidation data obtained or estimated from Martin et al. ${ }^{9}$ 
Figure S5. The structure of 53 quantitative PFAS.

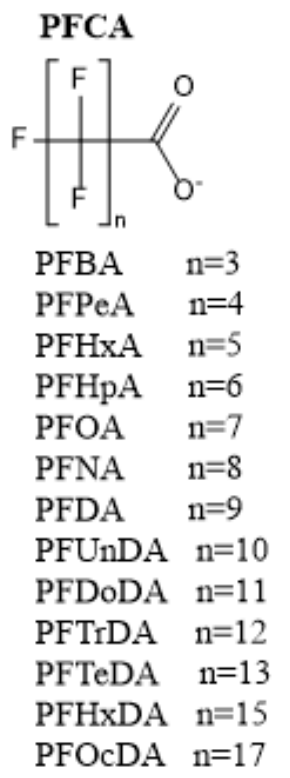

Perfluoroalkyl sulfonamides

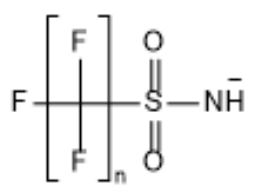

FBSA $n=4$

FHxSA $n=6$

FOSA $n=8$

FOSA derivatives

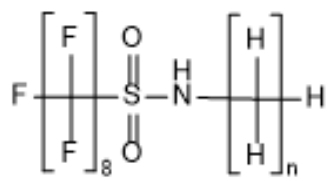

MeFOSA $n=1$

EtFOSA $\mathrm{n}=2$

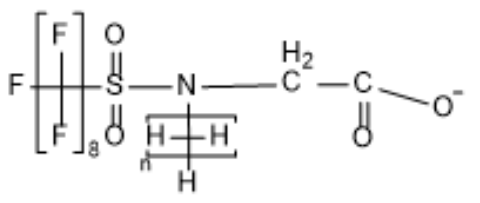

FOSAA $\mathrm{n}=0$

MeFOSAA $n=1$

EtFOSAA $n=2$

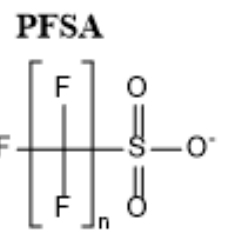

PFPrS $n=3$

PFBS $\mathrm{n}=4$

PFPeS $n=5$

PFHxS $\mathrm{n}=6$

PFHpS $n=7$

PFOS $n=8$

PFNS $n=9$

PFDS $\mathrm{n}=10$

PFDoS $\mathrm{n}=12$ n:2 FTSA<smiles>O=S(=O)([O-])CCC1(C(F)(F)F)CCCC1</smiles>

4:2 FTSA $n=4$

$6: 2$ FTSA $n=6$

8:2 FTSA $n=8$

10:2 FTSA $\mathrm{n}=10$

n:2 FTUA<smiles>O=C([O-])/C=C(\F)C(F)(F)C(F)(F)F</smiles>

6:2 FTUA $\mathrm{n}=5$

8:2 FTUA $\mathrm{n}=7$

10:2 FTUA $\mathrm{n}=9$ n:3 FTCA<smiles>O=C(O)CCC1(C(F)(F)F)CCCC1</smiles>

3:3 FTCA $\mathrm{n}=3$

4:3 FTCA $\mathrm{n}=4$

5:3 FTCA $\mathrm{n}=5$

$7: 3$ FTCA $\mathrm{n}=6$

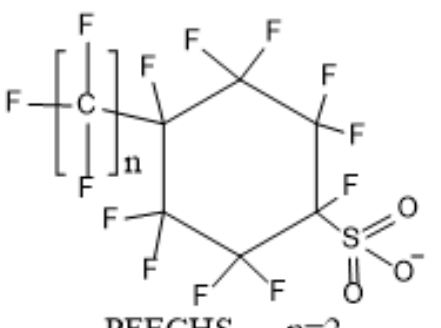

Novel Surfactants

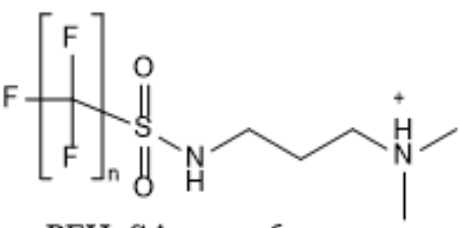

PFHxSAm $n=6$

PFOSAm $n=8$

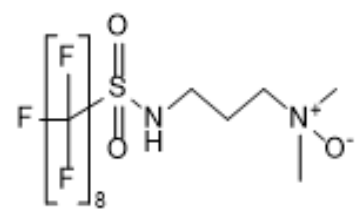

PFOSNO $\mathrm{n}=8$<smiles>C[N+](C)(CCCNS(=O)(=O)C(F)(F)C(F)(F)F)CC(=O)[O-]</smiles>

PFOSB $n=8$<smiles>C[N+](C)(C)CCCNS(=O)(=O)C(F)(F)C(F)(F)C(F)(F)C(F)(F)F</smiles>

PFHxSAmS $\mathrm{n}=6$

PFOSAmS $\mathrm{n}=8$
PFOANO $\mathrm{n}=7$

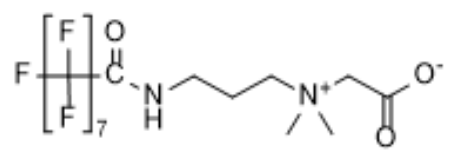

PFOAB $n=7$<smiles>C[N+](C)(CCCNS(=O)(=O)CCC(F)(F)C(F)(F)F)CCC[N+](C)(CC(=O)[O-])CC(=O)[OH2+]</smiles>

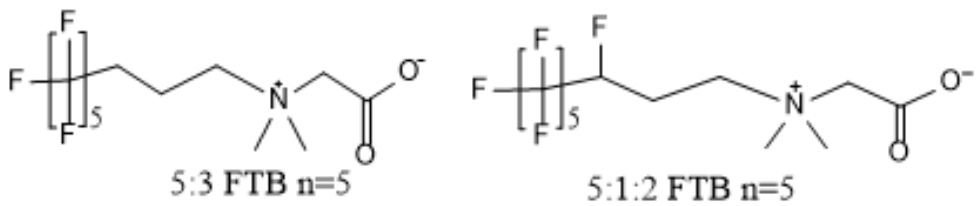

$5: 1: 2$ FTB $\mathrm{n}=5$ 
Figure S6. The concentrations of five types of PFAS in surface soils (a) and groundwater (b) from the four Canadian FTA sites.

Note: "up" refers to the upgradient area, "vicinity" refers to the vicinity of the FTA area, while "down" refers to the downgradient area.
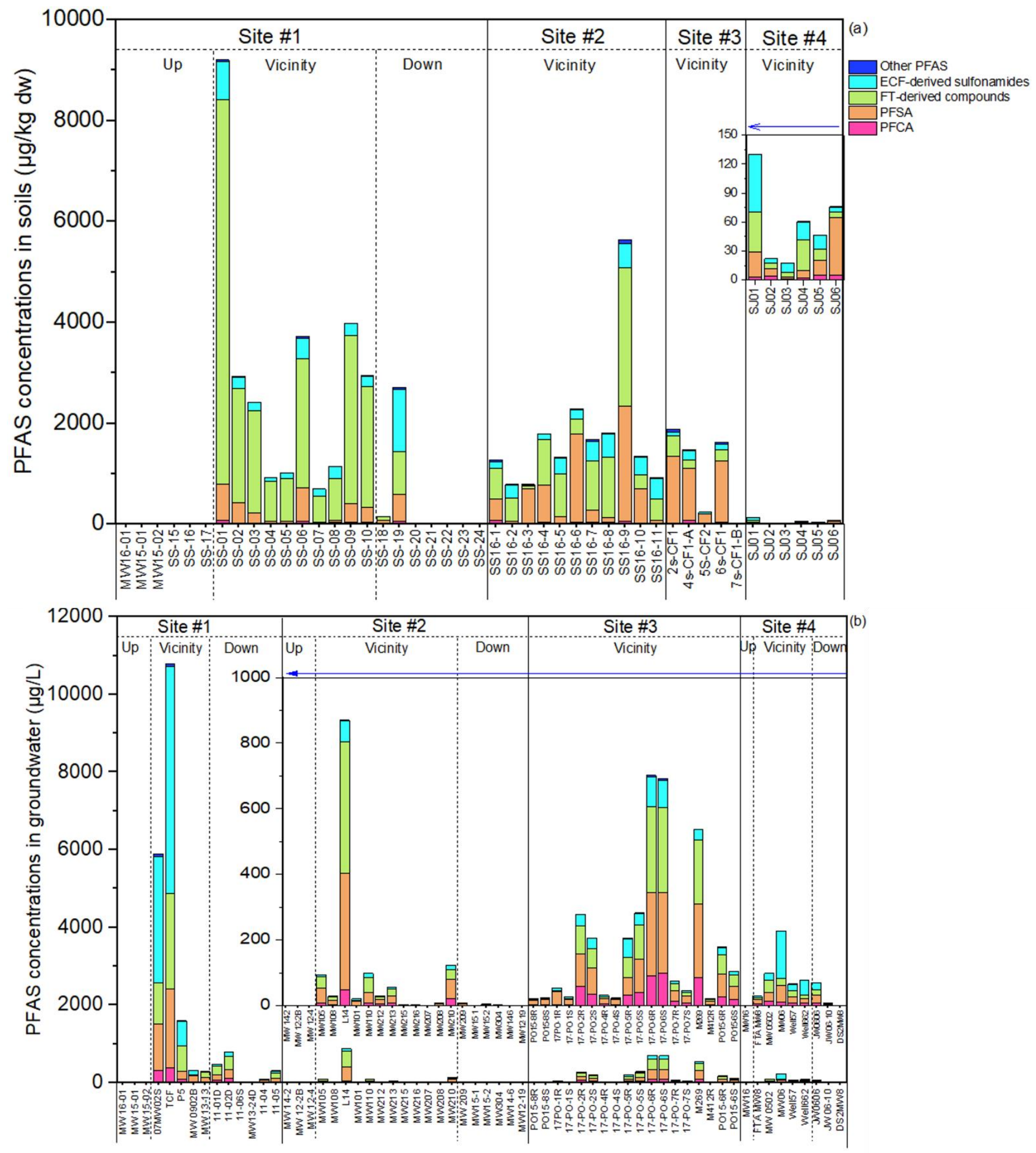
Figure S7. The fifteen highest PFAS measured in AFFF-impacted soils (a-d) and groundwater (e-h) in the vicinity of FTA area at the four FTA sites The zwitterionic and cationic precursors were marked with a red asterisk $\left(^{*}\right)$.
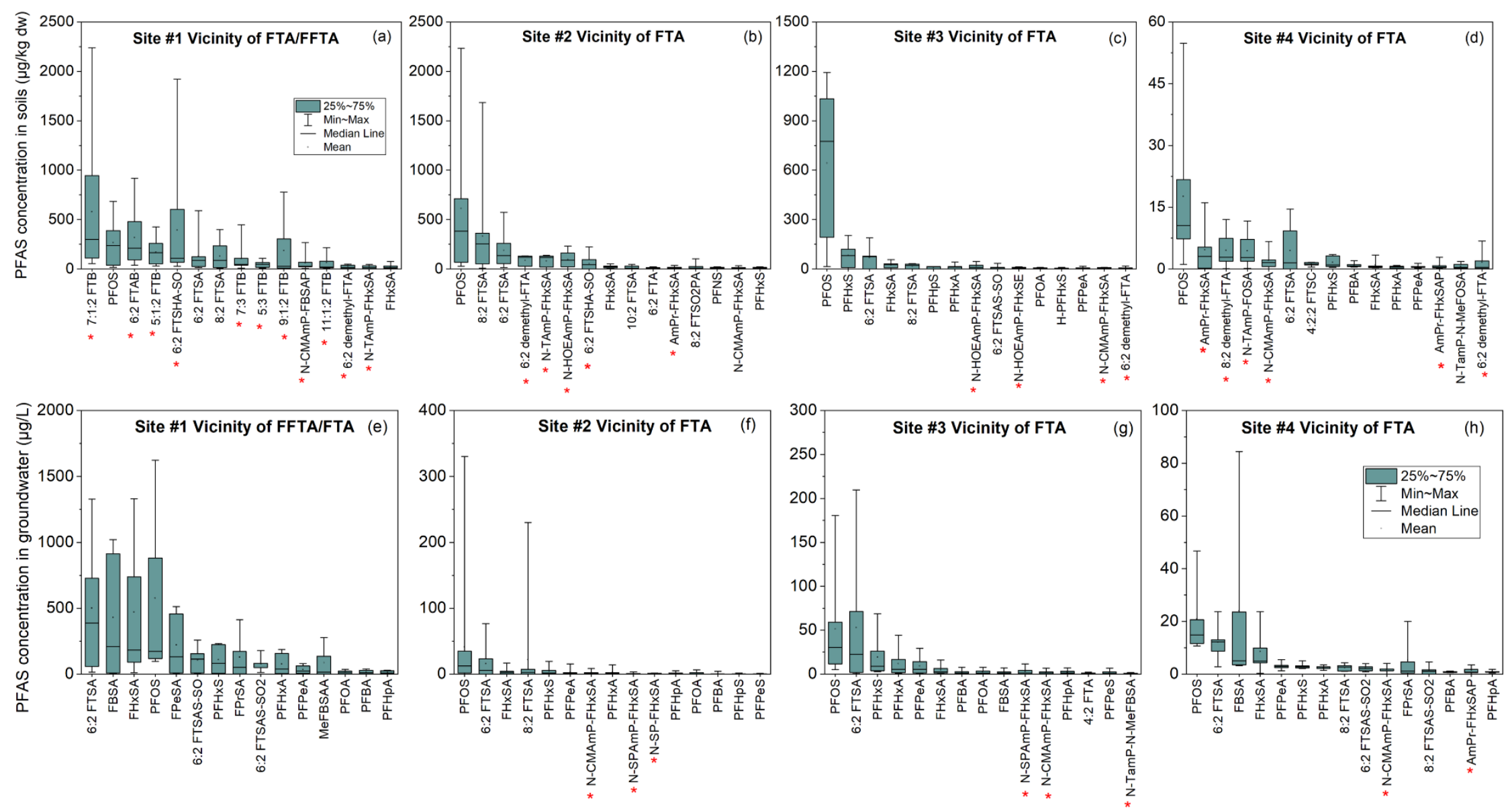
Figure S8. The PFAA concentrations in surface soil (a) and groundwater (b) samples from the four Canadian FTA sites.
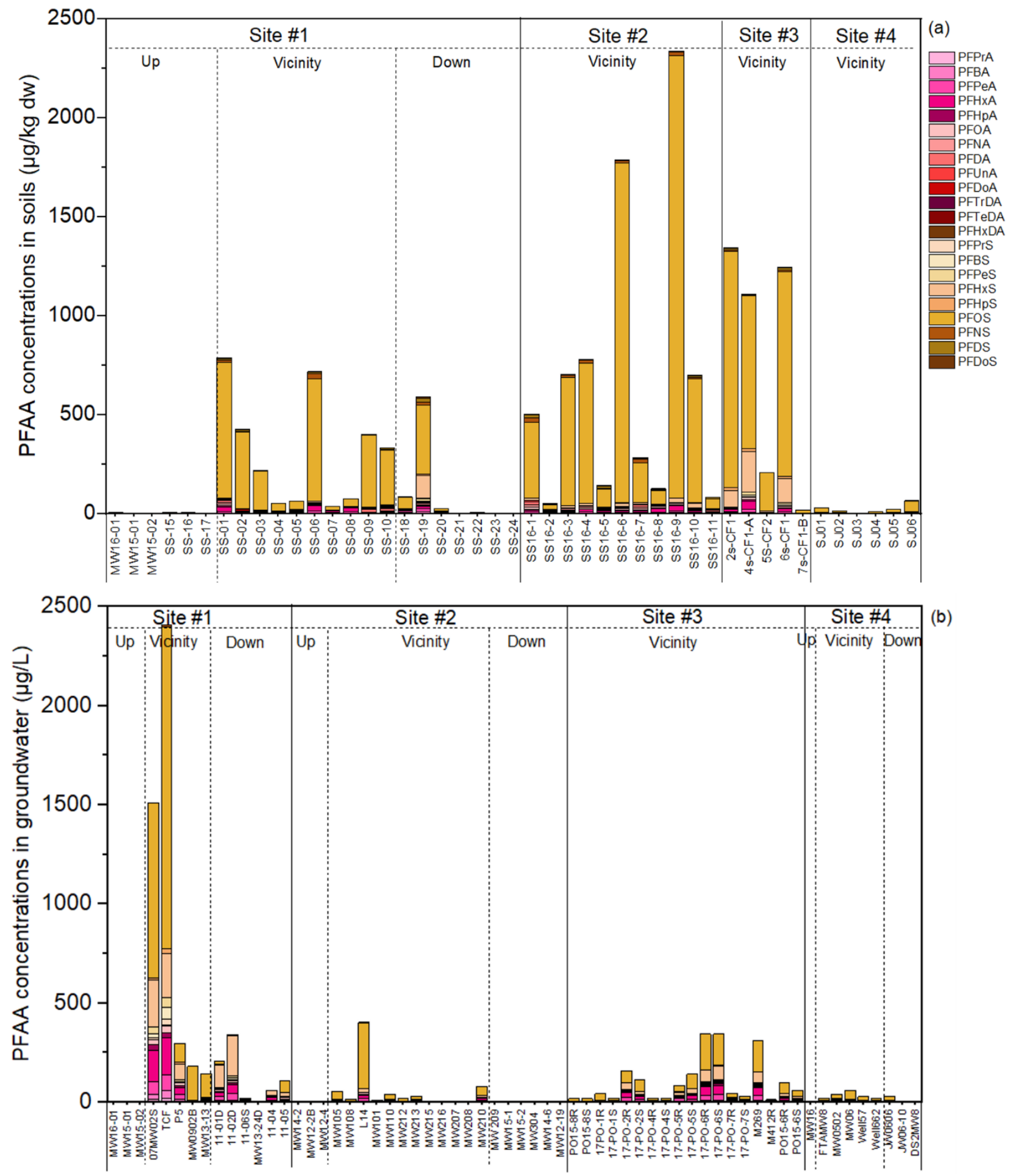
Figure S9. The profiles of ECF-derived sulfonamides in surface soil (a) and groundwater (b) samples from the four Canadian FTA sites.
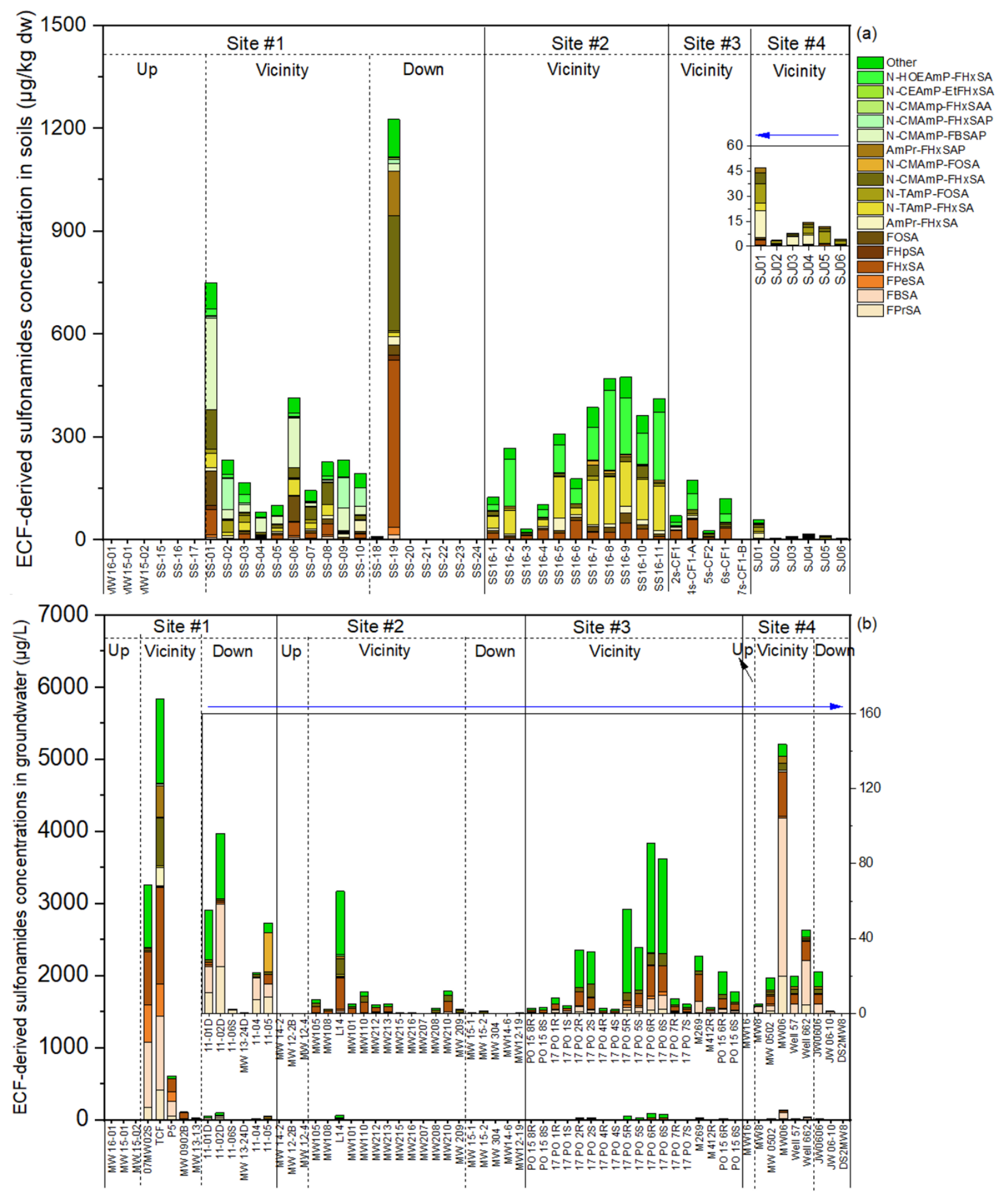
Figure S10. Likely in-situ transformation pathways of FT-derived precursors in source zone soils (a) and groundwater (c) of Site \#1 and the concentrations of the precursor and transformation products in soil (b) and groundwater (d) samples. Likely in-situ transformation pathways of ECF-derived sulfonamides in source zone soils of Site \#1 (e) and the concentrations of the precursor and transformation products in all samples (f).

(a)
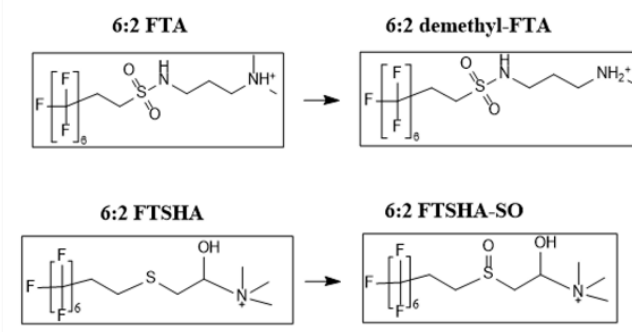

(b)
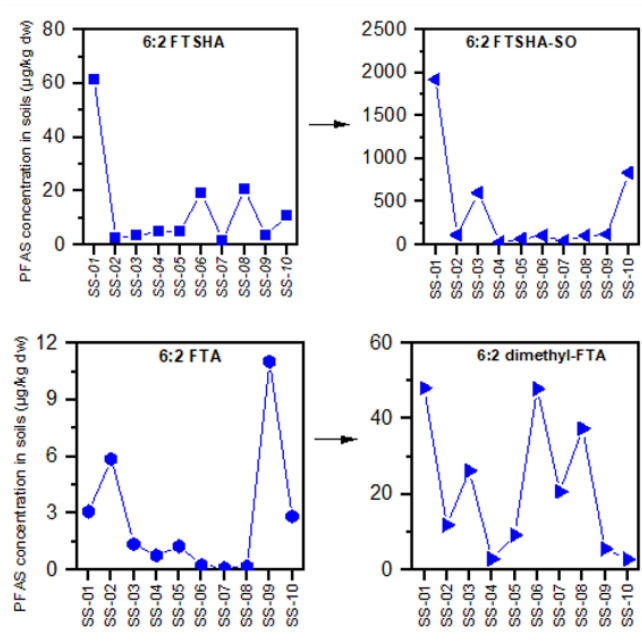

6:2 demethyl-FTA
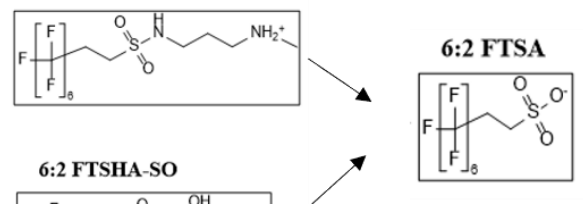

5:3 FTCA
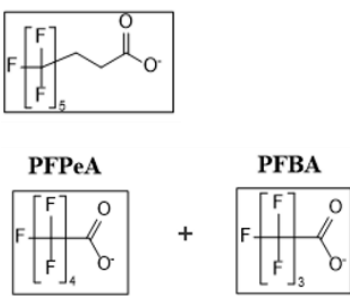

(c)

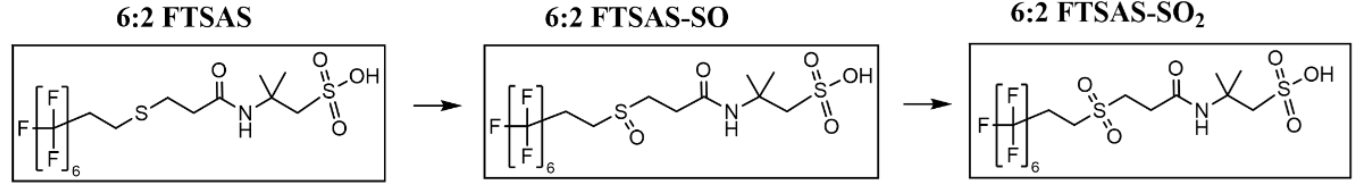

(d)
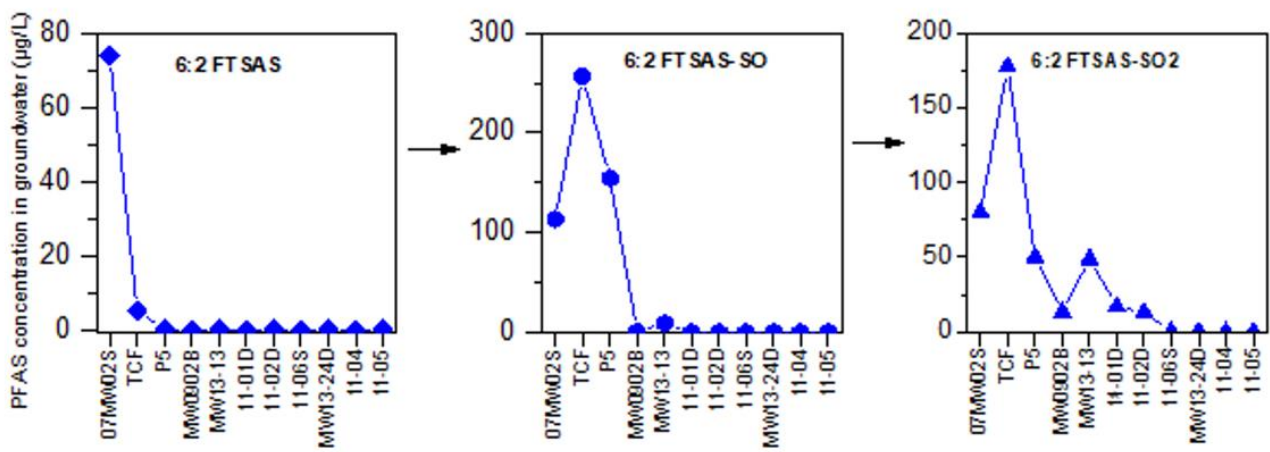
(e)
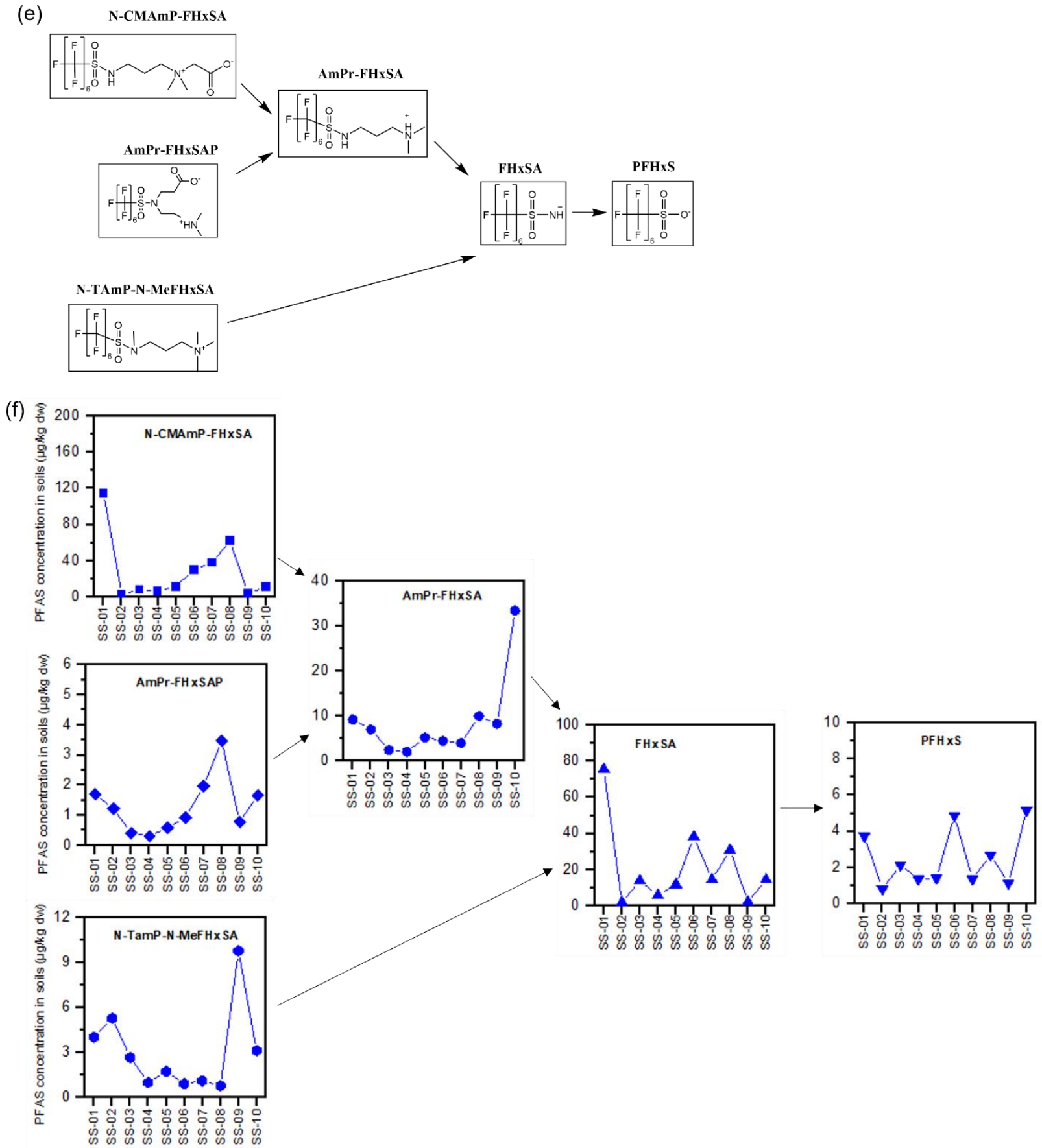
Figure S11. The profiles of FT-derived compounds in surface soil (a) and groundwater (b) samples from the four Canadian FTA sites.
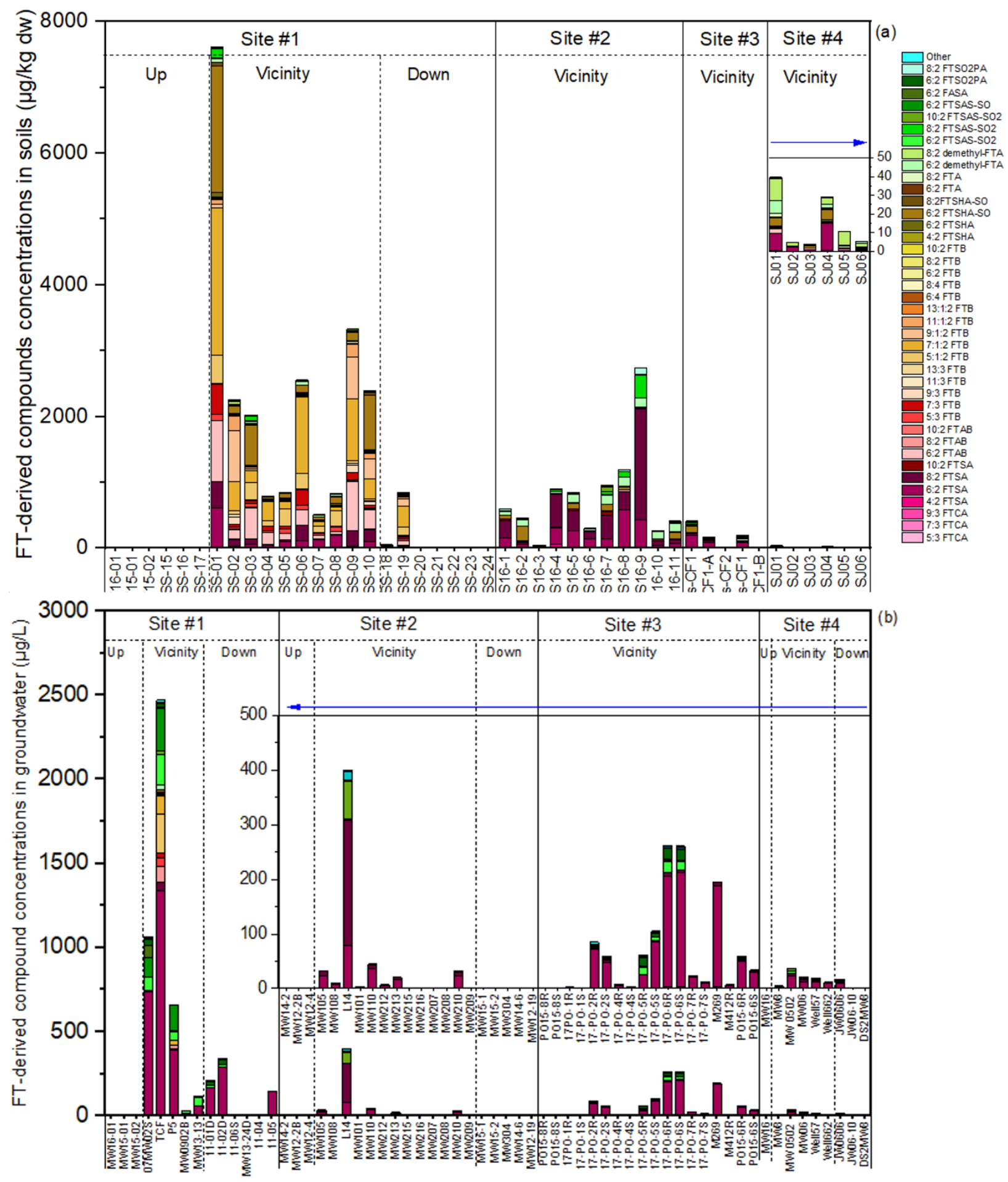
Figure S12. The changes in the concentrations of 15 PFAS (mainly detected in surface soils) over depths at five sampling locations (a) 4S, (b)2S, (c) $6 \mathrm{~S}$, (d)7S, (e) $5 \mathrm{~S}$ at site \#3. The five sample locations are shown in the scheme map (f).
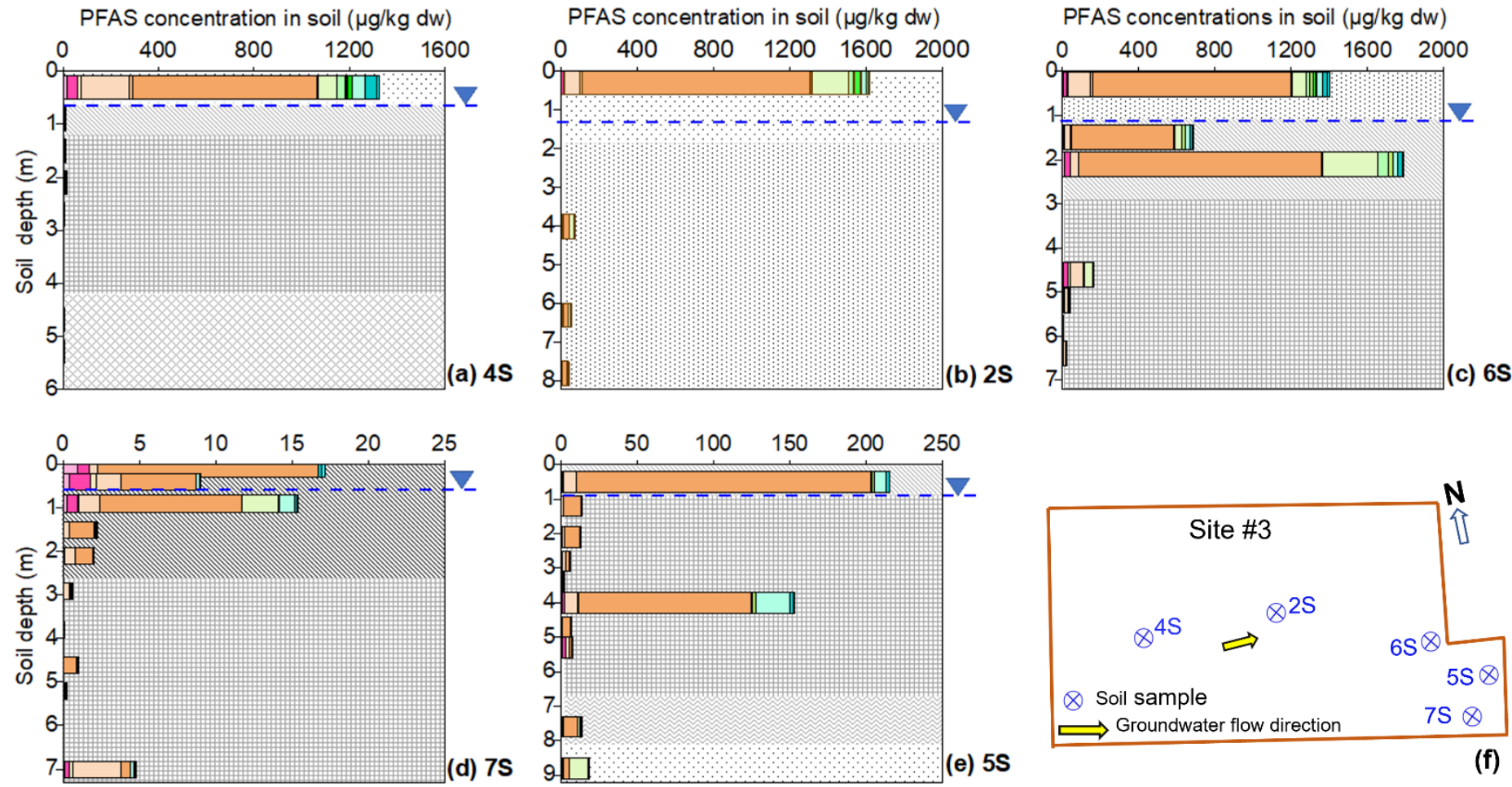

Sand

Silt and Sand

Silty clay

Clay

Sand and gravel

Sand, silt and gravel

d) $7 \mathrm{~s}$

(e) $5 \mathrm{~S}$ 
Figure S13. The molar concentration of both known and total precursors in (a-c) surface soil and (d-g) groundwater samples at the four Canadian FTA sites. The left bar represents the molar concentration of known precursors identified via UHPLC-HRMS through the target and suspect-screening methods, while the right bar shows the molar concentration of total precursors determined by TOP assay. The C3-C15 represented the carbon numbers of the known precursors (left bars) and the carbon number of PFCA produced from the precursors (right bars).

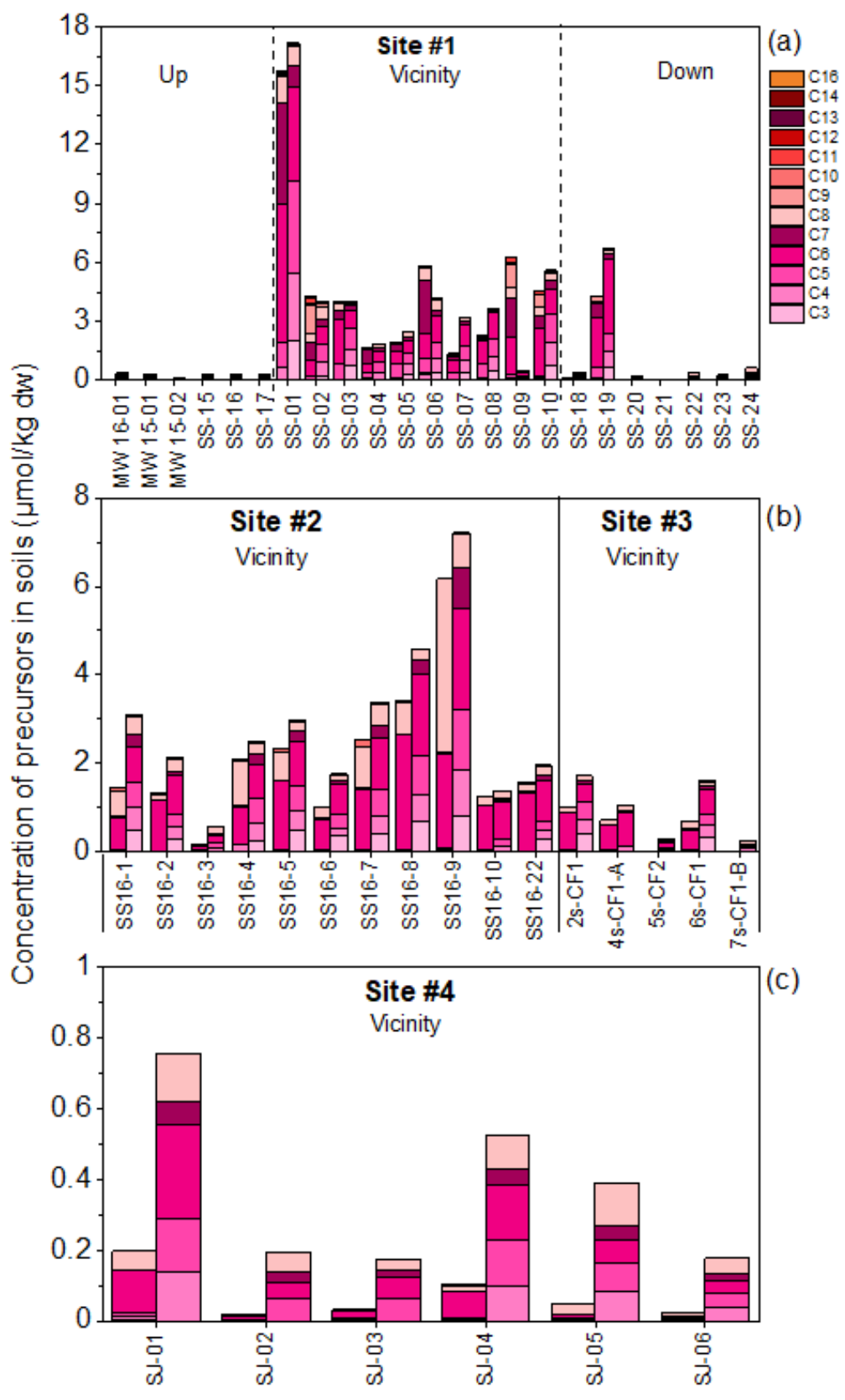



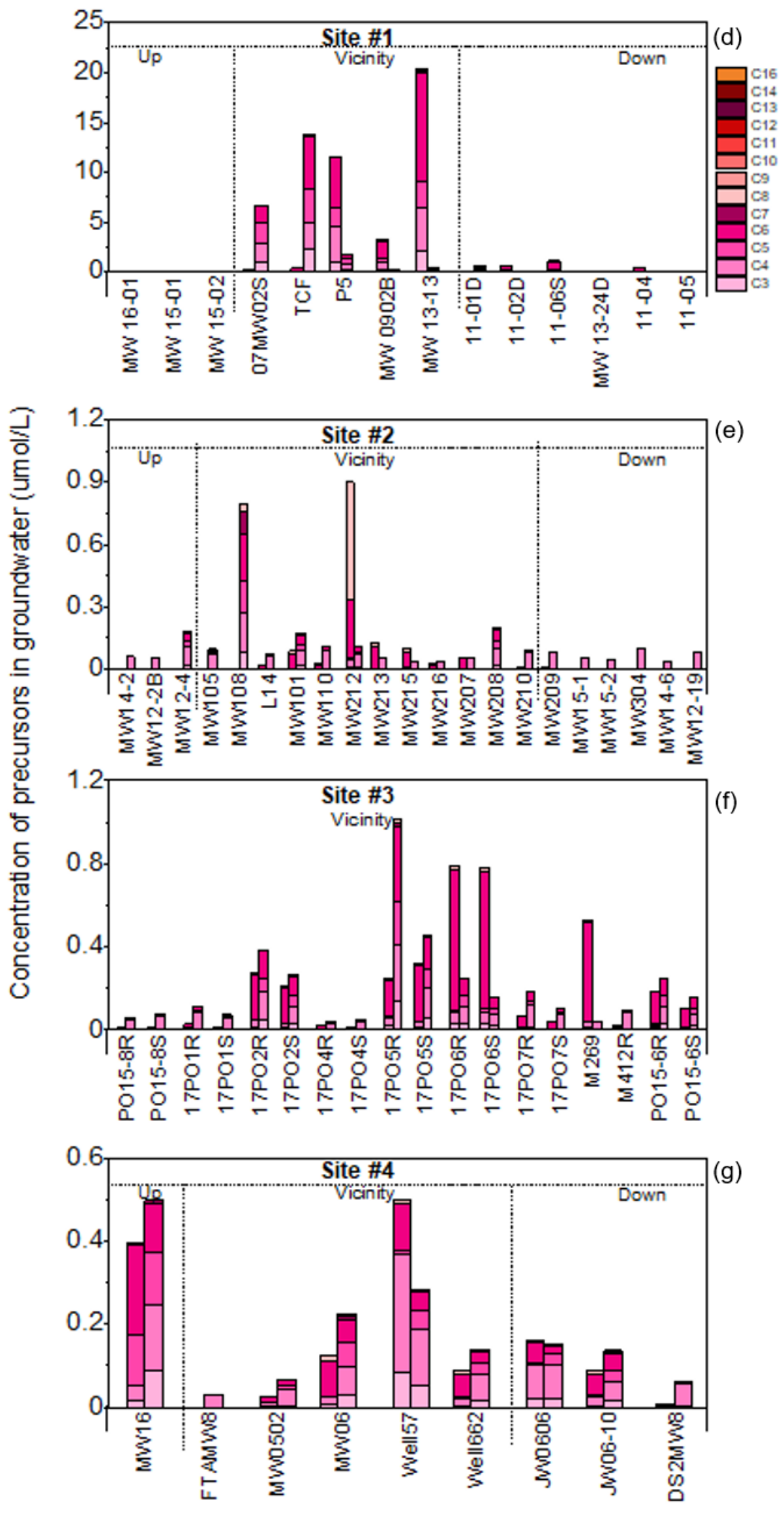
Figure S14. The molar fraction of unknown precursors in $\sum$ PFAS in both surface soil (a) and groundwater (b) when assuming molar PFCA yields of $80 \%, 100 \%$ and $120 \%$ from TOP.
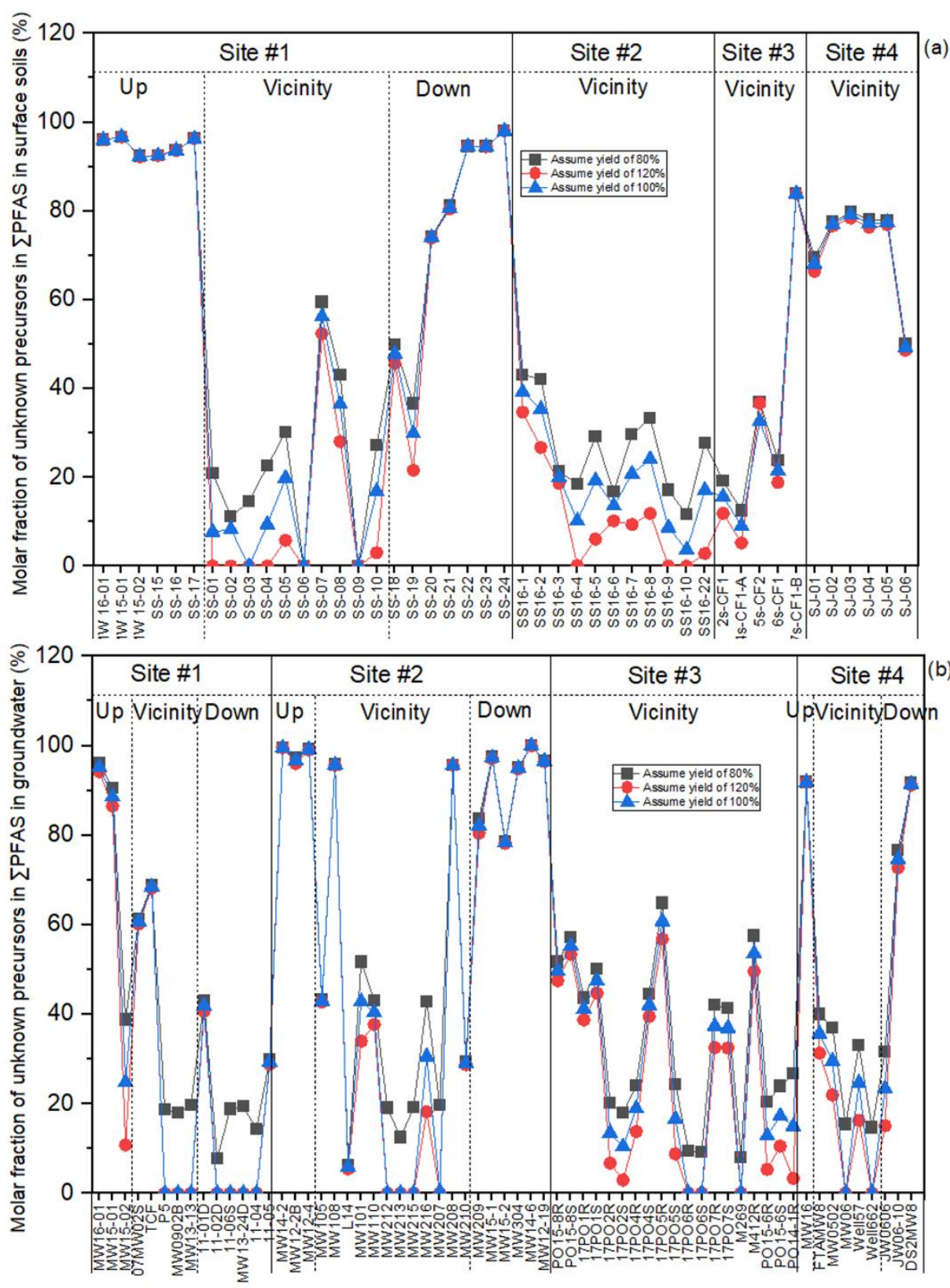


\section{Reference}

1. Munoz, G.; Ray, P.; Mejia-Avendaño, S.; Duy, S. V.; Do, D. T.; Liu, J.; Sauvé, S., Optimization of extraction methods for comprehensive profiling of perfluoroalkyl and polyfluoroalkyl substances in firefighting foam impacted soils. Anal. Chim. Acta 2018, 1034, 74-84.

2. Mejia-Avendaño, S.; Munoz, G.; Sauvé, S.; Liu, J., Assessment of the Influence of Soil Characteristics and Hydrocarbon Fuel Cocontamination on the Solvent Extraction of Perfluoroalkyl and Polyfluoroalkyl Substances. Anal. Chem. 2017, 89, (4), 2539-2546.

3. Munoz, G.; Desrosiers, M.; Duy, S. V.; Labadie, P.; Budzinski, H.; Liu, J.; Sauvé, S., Environmental Occurrence of Perfluoroalkyl Acids and Novel Fluorotelomer Surfactants in the Freshwater Fish Catostomus commersonii and Sediments Following Firefighting Foam Deployment at the Lac-Mégantic Railway Accident. Environ. Sci. Technol. 2017, 51, (3), 1231-1240.

4. Nickerson, A.; Maizel, A. C.; Kulkarni, P. R.; Adamson, D. T.; Kornuc, J. J.; Higgins, C. P., Enhanced extraction of AFFF-associated PFASs from source zone soils. Environ. Sci. Technol. 2020, 54, (8), 4952-4962.

5. Houtz, E. F.; Sedlak, D. L., Oxidative conversion as a means of detecting precursors to perfluoroalkyl acids in urban runoff. Environ. Sci. Technol. 2012, 46, (17), 9342-9.

6. Martin, D.; Munoz, G.; Mejia-Avendaño, S.; Duy, S. V.; Yao, Y.; Volchek, K.; Brown, C. E.; Liu, J.; Sauvé, S., Zwitterionic, cationic, and anionic perfluoroalkyl and polyfluoroalkyl substances integrated into total oxidizable precursor assay of contaminated groundwater. Talanta 2019, 195, 533-542.

7. Houtz, E. F.; Higgins, C. P.; Field, J. A.; Sedlak, D. L., Persistence of perfluoroalkyl acid precursors in AFFF-impacted groundwater and soil. Environ. Sci. Technol. 2013, 47, (15), 8187-8195.

8. Mejia-Avendaño, S.; Munoz, G.; Vo Duy, S.; Desrosiers, M.; Benoit, P.; Sauvé, S.; Liu, J., Novel Fluoroalkylated Surfactants in Soils Following Firefighting Foam Deployment During the Lac-Mégantic Railway Accident. Environ. Sci. Technol. 2017, 51, (15), 8313-8323.

9. Shoemaker, J.; Tettenhorst, D., Method 537.1: determination of selected per-and polyfluorinated alkyl substances in drinking water by solid phase extraction and liquid chromatography/tandem mass spectrometry (LC/MS/MS). National Center for Environmental Assessment, Washington, DC. 2018.

10. Schymanski, E. L.; Jeon, J.; Gulde, R.; Fenner, K.; Ruff, M.; Singer, H. P.; Hollender, J., Identifying Small Molecules via High Resolution Mass Spectrometry: Communicating Confidence. Environmental Science \& Technology 2014, 48, (4), 2097-2098.

11. Backe, W. J.; Day, T. C.; Field, J. A., Zwitterionic, cationic, and anionic fluorinated chemicals in aqueous film forming foam formulations and groundwater from U.S. military bases by nonaqueous largevolume injection HPLC-MS/MS. Environ. Sci. Technol. 2013, 47, (10), 5226-5234.

12. Munoz, G.; Duy, S. V.; Labadie, P.; Botta, F.; Budzinski, H.; Lestremau, F.; Liu, J.; Sauvé, S., Analysis of zwitterionic, cationic, and anionic poly- and perfluoroalkyl surfactants in sediments by liquid chromatography polarity-switching electrospray ionization coupled to high resolution mass spectrometry. Talanta 2016, 152, 447-456. 
13. Baduel, C.; Mueller, J. F.; Rotander, A.; Corfield, J.; Gomez-Ramos, M.-J., Discovery of novel perand polyfluoroalkyl substances (PFASs) at a fire fighting training ground and preliminary investigation of their fate and mobility. Chemosphere 2017, 185, 1030-1038.

14. Place, B. J.; Field, J. A., Identification of novel fluorochemicals in aqueous film-forming foams used by the US military. Environ. Sci. Technol. 2012, 46, (13), 7120-7127.

15. Weiner, B.; Yeung, L. W. Y.; Marchington, E. B.; D'Agostino, L. A.; Mabury, S. A., Organic fluorine content in aqueous film forming foams (AFFFs) and biodegradation of the foam component 6:2 fluorotelomermercaptoalkylamido sulfonate (6:2 FTSAS). Environ. Chem. 2013, 10, (6), 486-493.

16. D'Agostino, L. A.; Mabury, S. A., Identification of novel fluorinated surfactants in aqueous film forming foams and commercial surfactant concentrates. Environ. Sci. Technol. 2014, 48, (1), (1), 121-129. 17. Jensen, A. A.; Poulsen, P. B.; Bossi, R.; Miljøundersøgelser, D.; Technology, F., Survey and environmental/health assessment of fluorinated substances in impregnated consumer products and impregnating agents. Danish Environmental Protection Agency Copenhagen: 2008; Vol. 99.

18. Schultz, M. M.; Barofsky, D. F.; Field, J. A., Quantitative determination of fluorotelomer sulfonates in groundwater by LC MS/MS. Environ. Sci. Technol. 2004, 38, (6), 1828-1835.

19. Harding-Marjanovic, K. C.; Houtz, E. F.; Yi, S.; Field, J. A.; Sedlak, D. L.; Alvarez-Cohen, L., Aerobic Biotransformation of Fluorotelomer Thioether Amido Sulfonate (Lodyne) in AFFF-Amended Microcosms. Environ. Sci. Technol. 2015, 49, (13), 7666-74.

20. D'Agostino, L. A.; Mabury, S. A., Certain Perfluoroalkyl and Polyfluoroalkyl Substances Associated with Aqueous Film Forming Foam Are Widespread in Canadian Surface Waters. Environ. Sci. Technol. 2017, 51, (23), 13603-13613.

21. D'Agostino, L. A.; Mabury, S. A., Aerobic biodegradation of 2 fluorotelomer sulfonamide-based aqueous film-forming foam components produces perfluoroalkyl carboxylates. Environ. Toxicol. Chem. 2017, 36, (8), 2012-2021.

22. Barzen-Hanson, K. A.; Roberts, S. C.; Choyke, S.; Oetjen, K.; McAlees, A.; Riddell, N.; McCrindle, R.; Ferguson, P. L.; Higgins, C. P.; Field, J. A., Discovery of 40 Classes of Per- and Polyfluoroalkyl Substances in Historical Aqueous Film-Forming Foams (AFFFs) and AFFF-Impacted Groundwater. Environ. Sci. Technol. 2017, 51, (4), 2047-2057.

23. Mejia-Avendaño, S.; Duy, S. V.; Sauvé, S.; Liu, J., Generation of Perfluoroalkyl Acids from Aerobic Biotransformation of Quaternary Ammonium Polyfluoroalkyl Surfactants. Environ. Sci. Technol. 2016, 50, (18), 9923-9932.

24. Liu, Y.; Pereira, A. D. S.; Martin, J. W., Discovery of C5-C17 Poly- and Perfluoroalkyl Substances in Water by In-Line SPE-HPLC-Orbitrap with In-Source Fragmentation Flagging. Anal. Chem. 2015, 87, (8), 4260-4268.

25. Wang, N.; Szostek, B.; Buck, R. C.; Folsom, P. W.; Sulecki, L. M.; Gannon, J. T., 8-2 Fluorotelomer alcohol aerobic soil biodegradation: Pathways, metabolites, and metabolite yields. CHEM Chemosphere 2009, 75, (8), 1089-1096.

26. Rotander, A., Novel fluorinated surfactants tentatively identified in firefighters using liquid chromatography quadrupole time-of-flight tandem mass spectrometry and a case-control approach. Environ. Sci. Technol. 2015, 49, (4), 2434. 